\title{
Modelado de la respuesta dinámica del suelo en Granada con datos de métodos pasivos SPAC y HVSR.
}

\section{Trabajo Fin de Máster.}

\section{Máster Universitario en Estructuras.}

Alumno

Gerardo Velasco Ugalde

Tutores

Francisco Vidal Sánchez

Gerardo Alguacil de la Blanca

Granada, España.

26 de Septiembre de 2013. 



\section{Resumen.}

Los efectos de sitio tienen un gran peso en el cálculo de la peligrosidad sísmica. La amplificación de ondas por depósitos de suelo blando es una causa bien conocida del daño en estructuras durante terremotos, como claros ejemplos se cuentan los terremotos de Michoacán, México, en 1985 y Hyogo-ken Nambu, Japón, en 1995. La ciudad de Granada, ubicada en la región de mayor peligrosidad sísmica de España, ha sido afectada en numerosas ocasiones por terremotos moderados y en otras cuantas por eventos de gran magnitud. Con el fin de estudiar el efecto que tiene la geología local de Granada en la modificación de las ondas sísmicas de potenciales terremotos en la zona, se han hecho propagaciones de acelerogramas reales a través de modelos de suelo cuyas características son capaces de reproducir las condiciones reales de varios puntos en la ciudad. Dichos modelos de suelo son producto de una inversión conjunta de curvas de dispersión y curvas HVSR que a su vez han sido calculadas con los métodos SPAC y HVSR respectivamente. De esta manera se demuestra que la geología local de la ciudad es capaz de modificar el valor de los parámetros de movimiento sísmico de una manera arbitraria y para determinadas frecuencias en comparación a lo registrado en algún sitio sobre un afloramiento rocoso. 


\section{Agradecimientos.}

A mi familia por el apoyo durante mi formación académica. A los profesores Gerardo Alguacil de la Blanca y Francisco Vidal Sánchez por todas sus enseñanzas, por el tiempo dedicado y por los consejos que he recibido durante los últimos meses. Al profesor Manuel Navarro Bernal por los datos de las curvas de dispersión usadas en este trabajo. A Antonio García Jerez por las recomendaciones para obtener los modelos de velocidad. Al Instituto Andaluz de Geofísica y Prevención de Desastres Sísmicos por el apoyo con la instrumentación usada en las mediciones de ruido sísmico ambiental. Al Consejo Mexiquense de Ciencia y Tecnología por la beca otorgada que permitió mi estancia en España. 


\section{Índice.}

Resumen.

Agradecimientos. III

Índice. V

Lista de figuras.

VII

Lista de tablas IX

1 Introducción. 1

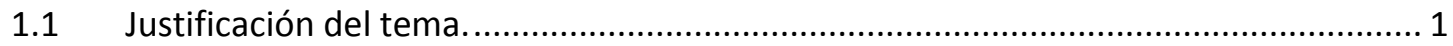

1.2 Terremotos que han presentado grandes efectos de sitio....................................... 3

1.3 Conceptos relacionados con el estudio de la respuesta de sitio. ................................. 4

1.4 Métodos de estimación de la respuesta de sitio. .................................................... 7

1.5 Aplicabilidad y antecedentes de los métodos HVSR y SPAC ...................................... 9

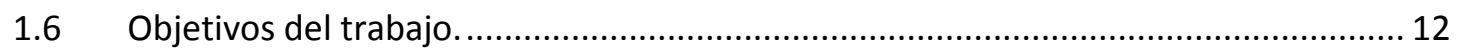

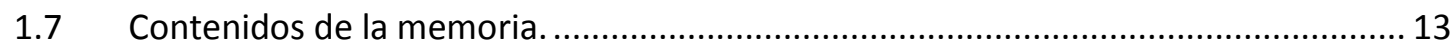

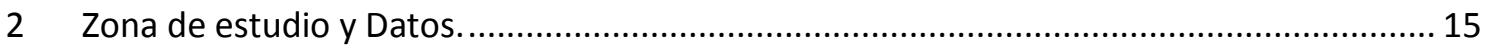

2.1 Características geológicas y geofísicas de la zona de estudio..................................... 15

2.2 Características de las medidas de microtremor en arreglos........................................ 21

2.3 Características de las medidas de microtremor con una estación. .............................. 22

3 Cálculo de las curvas de dispersión y curvas HVSR. ...................................................... 27

3.1 Método de Auto correlación espacial modificada SPAC ........................................... 27

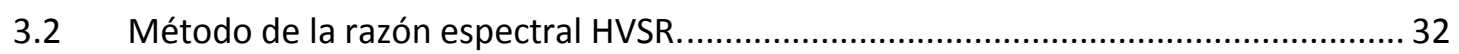

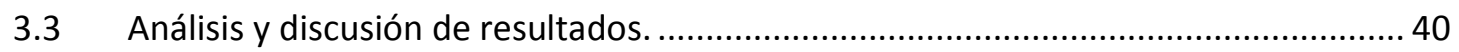


4 Inversión de los modelos de velocidad mediante curvas de dispersión y curvas HVSR. .... 42

4.1 Método de inversión.

4.2 Análisis y discusión de los resultados de la inversión conjunta..................................46

5 Cálculo de la respuesta dinámica del suelo en la ciudad de Granada. .............................. 49

5.1 Método para obtener la respuesta del suelo de una estructura del terreno.............. 49

5.2 Parámetros de movimiento sísmico del suelo............................................................ 52

5.3 Características de la respuesta dinámica del suelo................................................... 55

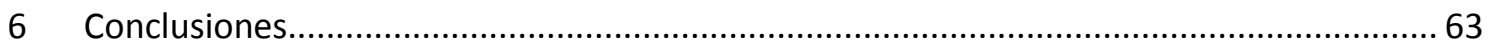

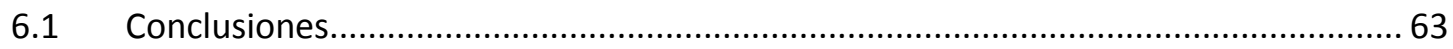

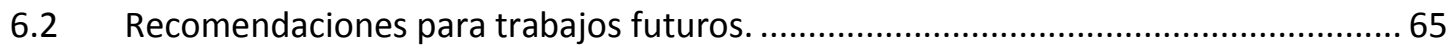

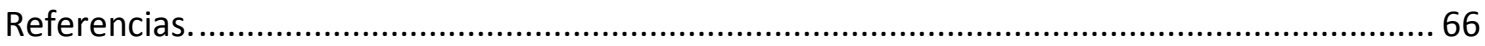

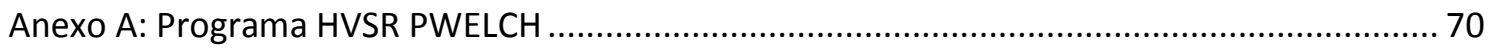

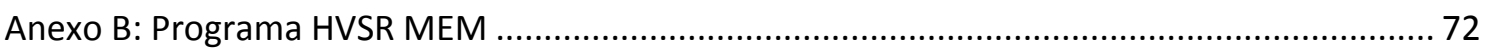




\section{Lista de figuras.}

Figura 2.1 Localización de los terremotos más energéticos ocurridos los últimos 500 años en la cuenca de Granada y de las fallas activas y probablemente activas con su tasa de deslizamiento (Sanz de Galdeano et al., 2003). El área urbana de Granada se muestra en blanco. Los epicentros y magnitudes de los terremotos históricos se asignaron a partir de datos macro sísmicos.

Figura 2.2 Esquema Geológico de la cuenca de Granada (Sanz de Galdeano, et al. 2001). En amarillo se muestran las áreas con materiales plio-cuaternarios, donde está ubicada la ciudad de Granada.

Figura 2.3 Representación gráfica del valor Vs medio en Granada (Valverde, 2010).

Figura 2.4 Plano de profundidad del material competente (Hernández del Pozo, 1998)

Figura 2.5 Microzonificación elaborada a partir de velocidades Vs por Cheddadi (2001) 20

Figura 2.6 Microzonificación sísmica de Granada basada en períodos predominantes del suelo (Cheddadi, 2001). 20

Figura 2.7 Localización de los puntos de toma de medidas de SPAC y principales unidades geológicas superficiales de la ciudad de Granada (Navarro et al, 2011).

Figura 2.8 Despliegue del array en Fuentenueva que muestra los dispositivos de medición (Feriche, 2012)

Figura 2.9 Mapa de la Ciudad de Granada con los sitios de las medidas de microtremor para usar la técnica HVSR.

Figura 2.10 Estación sísmica y sensor sísmico Taurus.

Figura 2.11 Estacionaridad de la respuesta de la razón HVSR en 60 minutos para el sitio de la Calle Náyades. Las gráficas se han calculado con el programa Geopsy.

Figura 2.12 Estacionaridad de la respuesta de la razón espectral HVSR en 90 minutos para el sitio de la Calle Aixa la Horra. Las gráficas se han calculado con el programa Geopsy. 26 
Figura 3.1 Curvas de dispersión obtenidas en Granada a) Aynadamar; b) Zaidín; c) Beiro; d) Bola de Oro; e) Fuentenueva; f) Estadio; g)Chana; h) Náyades; i)Ciencias; j) Granada 74.

Figura 3.2 Razones espectrales HVSR obtenidas con el programa HVSR Pwelch en Bola de oro (izquierda) y Calle Aixa la Horra (derecha) 35

Figura 3.3 Razones espectrales HVSR obtenidas con programa HVSR MEM. a) Fuentenueva b) Calle Aixa la Horra c) Escuela de Caminos d) Campo de los Mártires. 36

Figura 3.4 Ejemplo de la función Taper al 20\% en el dominio del tiempo.

Figura 3.5 Ejemplo de señal en Geopsy con algoritmo de anti-disparo, en verde se muestran las ventanas útiles para el cálculo de la razón espectral HVSR. Sitio Granada 74. 38

Figura 3.6 Razones espectrales HVSR obtenidas con el programa Geopsy. a) Aynadamar; b) Zaidín; c) Beiro; d) Bola de Oro; e) Fuentenueva; f) Estadio; g) Chana; h) Náyades; i) Ciencias; j) Granada 74.

Figura 4.1 Ejemplos de Inversión de la curva de dispersión con valor de desajuste hecha con dinver de Geopsy. Sitio Beiro (izquierda) y Bola de Oro (derecha).

Figura 4.2 Ejemplos de Inversión de la curva $H / V$ con valor de desajuste hecha con dinver de Geopsy. Sitio Fuentenueva (izquierda) y Náyades (derecha).

Figura 4.3 Modelos de Velocidad Vs obtenidos de la inversión conjunta de las curvas de dispersión y la razón HVSR a) Aynadamar; b) Zaidín; c) Beiro; d) Bola de Oro; e) Fuentenueva; f) Estadio; g)Chana; h) Náyades; i)Ciencias; j) Granada 74.

Figura 5.1 Acelerogramas medidos en la estación RNR del terremoto de Campano-Lucano, Italia.

Figura 5.2 Acelerogramas medidos en la estación GSA del terremoto de L'Aquila, Italia. 50

Figura 5.3 Acelerogramas del terremoto de Campano-Lucano propagados unidimensionalmente con el programa Degtra. Sitio Chana. 51

Figura 5.4 Acelerogramas del terremoto de L'Aquila propagados unidimensionalmente con el programa Degtra. Sitio Ciencias. 
Figura 5.5 Comparación de los valores de PGA y PGV para los terremotos de Campano-Luca y L'Aquila en los 10 sitios de estudio y en el de referencia.

Figura 5.6 Comparación de los valores de CAV para los terremotos de Campano-Luca y L'Aquila en los 10 sitios de estudio y en el de referencia. 58

Figura 5.7 Comparación de los valores de Al para los terremotos de Campano-Luca y L'Aquila en los 10 sitios de estudio y en el de referencia. 58

Figura 5.8 Espectros de respuesta de aceleración del terremoto de Campano-Lucano en los 10 sitios de estudio y el de referencia. Puede apreciarse gran diferencia entre los valores en suelo (10 sitios ) y en roca (el de referencia). 60

Figura 5.9 Espectros de respuesta de aceleración del terremoto de L'Aquila en los 10 sitios de estudio y el de referencia. Puede apreciarse la gran diferencia entre los valores en suelo (10 sitios ) y en roca (el de referencia). 60

Figura 5.10 Espectros de input de energía del terremoto de Campano-Lucano y el de referencia. Obsérvese la gran diferencia entre los valores en suelo (10 sitios ) y en roca (el de referencia). 61

Figura 5.11 Espectros de input de energía del terremoto de L'Aquila y el de referencia. Obsérvese la gran diferencia entre los valores en suelo (10 sitios ) y en roca (el de referencia). 62

\section{Lista de tablas.}

Tabla 3.1 Resultados del estudio de la razón espectral HVSR en todos los sitios. Incluyendo hora de medición, coordenadas del sitio, Frecuencia dominante, Periodo dominante y Amplitud de la razón HVSR.

Tabla 4.1 Resultados de la Inversión conjunta hecha con "dinver" de Geopsy. Se presenta el valor de la función de desajuste (Misfit) del mejor modelo por cada sitio.

Tabla 5.1 Resultados de PGA, PGV, Al y CAV para los terremotos de Campano-Lucano y L'Aquila en los 10 sitios de estudio y en la referencia.

Tabla 5.2 Media logarítmica de los parámetros PGA, PGV, Al y CAV del conjunto de acelerogramas de terremotos euro-mediterráneos analizados por Aguacil y Vidal (2012)....... 59 
Modelado de la respuesta dinámica del suelo en Granada con datos de métodos pasivos SPAC y HVSR. 



\section{Introducción.}

\subsection{Justificación del tema.}

La ciudad de Granada se encuentra ubicada en la Comunidad Autónoma de Andalucía, la cual es considerada la región de mayor peligrosidad sísmica de España según su actividad sísmica. Los datos de sismicidad histórica revelan que la ciudad de Granada ha sido afectada en varias ocasiones por terremotos locales o muy cercanos. Por ejemplo podemos mencionar los del 24 de Abril de 1431 y 4 de Julio de 1526 en la propia Granada, los del 27 de octubre de 1806 y 31 de mayo de 1911 en Santa Fe, el del 25 de diciembre de 1884 en Arenas del Rey y el del 19 de abril de 1656 de Albolote (Vidal, 1986). Debido a esto, la norma de construcción sismorresistente española NCSE-02 considera a la ciudad de Granada como la de mayor peligrosidad sísmica de toda España, con una aceleración sísmica básica de $0.24 \mathrm{~g}$ para un periodo de retorno de 475 años. El estudio las características del movimiento sísmico del terreno en diferentes partes de la ciudad de Granada adquiere un especial interés en la medida de poder garantizar la toma de medidas preventivas, de mitigación y de preparación para una respuesta adecuada ante un eventual terremoto.

El riesgo sísmico es el grado de pérdidas esperadas debidas a un terremoto de una magnitud particular y un periodo de exposición determinado. Para determinar el riesgo sísmico de cierto emplazamiento se debe evaluar la peligrosidad sísmica en primera instancia. Esta última se define como la probabilidad de superación de un cierto valor de intensidad del movimiento del suelo en un punto en concreto y durante un periodo de tiempo determinado.

Para evaluar la peligrosidad sísmica en un emplazamiento se deben tener en cuenta tres factores principales:

1) Las características de las fuentes que podrían generar eventos sísmicos: El mecanismo de la falla controla la distribución de la energía radiada en el espacio y el tiempo. Esta energía a su vez se distribuye en forma de ondas sísmicas que viajan a través de la corteza terrestre.

2) Los efectos de propagación de la energía sísmica por la corteza: Las ondas sísmicas son afectadas por diferencias en las propiedades mecánicas dentro del medio por donde viajan, presentándose fenómenos de reflexión, refracción y difracción.

3) Los efectos locales o de sitio: Por último, cuando las ondas pasan cerca de la superficie sus propiedades espectrales y temporales sufren cambios significativos. Este proceso 
no es idéntico para los diferentes puntos en la superficie y depende de las condiciones locales de cada sitio.

Los efectos de sitio tienen un gran peso en el cálculo de la peligrosidad sísmica, la amplificación de ondas por depósitos de suelo blando cerca o en la superficie es una causa bien conocida del daño en estructuras durante terremotos, como por ejemplo el caso del terremoto de Michoacán, México en 1985 (Celebi et al., 1987).

Estudios previos sugieren que en zonas de suelo blando los efectos de sitio son más relevantes que aquellos relacionados a la fuente, aun en campo cercano (Borcherdt, 1970). Como ejemplo de lo anterior se cita el informe del terremoto de San Francisco de 1906 hecho por la Comisión Estatal de Investigación Sísmica de California, "La cantidad de daño producida por el terremoto de la bahía de San Francisco de 1906 depende principalmente de la condición geológica del suelo. En donde la superficie es roca se produjo daño limitado; la mayor violencia del terremoto se manifestó sobre la tierra ganada al mar".

Muchas ciudades, como p. e. Granada, están emplazadas sobre estructuras sedimentarias que pueden provocar una significativa amplificación de las ondas sísmicas. La presencia de estos terrenos blandos implica un incremento de los valores máximos del movimiento en términos de aceleración, velocidad y desplazamiento (PGA, PGV, PGD), una variación en los valores alcanzados de estos parámetros en diferentes rangos de frecuencias, y también un aumento en la duración de la sacudida sísmica (SISMOSAN, 2007).

Sin embargo, la caracterización de la sacudida del suelo en términos de las condiciones locales no es una tarea sencilla por la diversidad de los materiales geológicos y las formas irregulares de las estructuras del suelo formadas por erosión, intemperización, sedimentación y otros procesos geológicos cercanos a la corteza terrestre (Aki, 1993).

Por tales motivos se propone un estudio de los posibles efectos de sitio que pueden ser causados por la estructura de suelo de la ciudad de Granada. Haciendo uso para ello de técnicas pasivas y de bajo costo que sean adecuadas para zonas urbanas con actividad sísmica moderada, como lo son los métodos SPAC y HVSR. De esta forma se pretende aportar información valiosa para mejorar la determinación de la peligrosidad sísmica en la ciudad. 


\subsection{Terremotos que han presentado grandes efectos de sitio.}

Han sido numerosos los terremotos en donde la influencia de los efectos de sitio ha aumentado su poder destructivo y su capacidad para dañar estructuras. A continuación se discuten algunos de ellos.

\section{Ciudad de México, México, 1985.}

La ciudad de México se encuentra en una zona estructural y geológica única (Vázquez, et al. 2011), sobre todo en lo concerniente a generar fenómenos de amplificación geométrica y dinámica. El terremoto de Michoacán de 1985, ocurrido la madrugada de 19 de Septiembre con magnitud Ms = 8.1, ocasionó grandes daños en la zona lacustre del valle de México. Aunque su epicentro se localizó a $400 \mathrm{~km}$ de la Ciudad México, el terremoto causó la muerte de más de 10,000 personas y la destrucción de unas 1,000 construcciones (Beck y Hall, 1986). Los registros de movimiento fuerte obtenidos en la Ciudad de México durante este terremoto mostraban gran amplitud en estaciones localizadas sobre material lacustre del antiguo lago de Texcoco. Dichos suelos se caracterizan por ser blandos, compresibles, tener gran contenido de agua y contar con valores de velocidad de onda de cizalla bajos. En comparación, en la zona denominada de la colina, caracterizada por tener suelos duros y poco compresibles, no se presentaron amplificaciones importantes. La amplificación espectral relativa fue de cerca de 50 para el rango de frecuencias entre 0.2 y $0.7 \mathrm{~Hz}$. (Singh et al., 1988).

\section{Spitak, Armenia, 1989.}

Otro terremoto en donde se observaron efectos locales importantes fue el del 7 de diciembre de 1989 de Spitak, Armenia. En la ciudad de Leninakan, localizada en una cuenca aluvial a 32 $\mathrm{km}$. del epicentro, se observó gran cantidad de daño mientras que en ciudades como Kirovakan, ubicada sobre una región de roca compacta a $25 \mathrm{~km}$ del epicentro, el daño fue significativamente menor. Los estudios hechos con registros de las réplicas del terremoto mostraron amplificaciones relativas de hasta 30 veces en un intervalo de frecuencias de 0.4 a $2.0 \mathrm{~Hz}$. (Borcherdt et. al., 1989).

\section{Loma Prieta, Estados Unidos de América, 1989.}

Tuvo ocurrencia el 17 de Octubre de 1989 con una magnitud Ms=7.1. Se observó una gran dependencia entre las amplitudes del movimiento del suelo y la geología local a partir de 50 
$\mathrm{km}$. desde el epicentro. Esta diferencia fue notable en el distrito de Marina, específicamente en las calzadas entre San Francisco y Oakland, en donde se obtuvieron diferencias del $200 \%$ en el valor de la aceleración en comparación con otros puntos de la ciudad ubicados sobre materiales compactos (Borcherdt, 1990).

\section{Hyogo-ken Nambu, Japón, 1995.}

El 17 de enero de 1995 la ciudad de Kobe sufrió el terremoto catalogado como el más destructivo en Japón desde 1923, causando más de 5,000 muertes y pérdidas por unos doscientos mil millones de dólares (Somerville, 1995). La velocidad pico en campo cercano registrada en dos sitios sobre suelo sedimentario fue muy superior al registrado en los sitios sobre roca adyacentes (Toki et. al., 1995).

\section{Izmit, Turquía, 1999.}

Turquía experimentó dos grandes terremotos que rompieron la falla del Norte de Anatolia los días 17 de agosto $\left(\mathrm{M}_{\mathrm{w}}=7.4\right)$ y 12 de noviembre $\left(\mathrm{M}_{\mathrm{w}}=7.1\right)$ de 1999. Adapazari, la capital de la provincia de Sakarya, sufrió la pérdida del $2 \%$ de su población y el $12 \%$ de sus edificios colapsaron. El nivel de daño fue sorprendentemente bajo en la sección sur de la ciudad, situada sobre suelos duros y superficiales, a pesar de que la distancia a la falla era tan solo de 5 km. En contra posición, la concentración de daño incrementó rápidamente hacia el norte, en donde los suelos son formados por sedimentos aluviales de gran espesor. La cantidad de construcciones dañadas excedió el $22 \%$ en los distritos centrales en donde los suelos aluviales tienen espesores de entre 125 y 200 m (Sadik et al., 2002).

\subsection{Conceptos relacionados con el estudio de la respuesta de sitio.}

\section{Amplificación espectral y Periodo dominante del suelo.}

La geología y topografía de un sitio en particular son capaces de alterar las características de las ondas sísmicas de manera importante y arbitraria. Los efectos de amplificación de la señal sísmica pueden deberse a dos diferentes mecanismos. 1) Amplificación geométrica y 2) Amplificación dinámica. La primera está relacionada con el contraste de impedancia entre el suelo y el basamento rocoso. Esto produce confinamiento de energía en las capas de suelo, influyendo así en el aumento de la amplitud de las ondas, en el contenido frecuencial de la señal y en la prolongación de la duración de la sacudida (Sánchez Sesma, 1987). La 
amplificación dinámica también se conoce como efecto de resonancia y considera la frecuencia con la que vibra el suelo al ser excitado por las ondas sísmicas, o frecuencia natural del depósito sedimentario. Así, el sustrato sedimentario responde con una mayor amplitud a aquellas frecuencias de las ondas sísmicas que coinciden o están muy está próximas a la frecuencia natural del suelo, lo que produce una amplificación del movimiento del suelo en torno a esas frecuencias

En la ingeniería sísmica es muy importante el estudio del movimiento del suelo en un rango de frecuencias que va de $0.1 \mathrm{~Hz}$. a $20 \mathrm{~Hz}$. Cabe recalcar que los valores de velocidad de propagación de ondas sísmicas S cerca de la superficie terrestre van de menos de $100 \mathrm{~m} / \mathrm{s}$ a más de $2000 \mathrm{~m} / \mathrm{s}$. Esto significa que las longitudes de onda asociadas a este movimiento varían de 10 metros a 20 kilómetros. Cuando las dimensiones de las estructuras geológicas en superficie son comparables con estas longitudes de onda predominantes, son de esperar cambios significativos en el movimiento sísmico en las irregularidades y sus proximidades. (Luzón et. al., 2002).

\section{Fuentes de excitación para el estudio de la respuesta de sitio.}

Es importante revisar de antemano cuáles son las fuentes de información que se usan para la obtención de la respuesta de sitio. En este caso la información puede provenir del registro en estaciones sísmicas de terremotos, explosiones y ruido sísmico ambiental.

\section{Terremotos.}

Parte de la energía liberada durante un terremoto se disipa en fenómenos no elásticos en la zona de ruptura y parte se propaga en forma de ondas elásticas que hacen vibrar el terreno. Resulta lógico usar registros de movimiento fuerte de suelo como fuente de información para obtener los efectos de sitio en cierto emplazamiento. Sin embargo, en regiones donde la sismicidad es moderada o se carece de redes de sismógrafos densas, la información puede ser escasa y se tiene que recurrir a otras fuentes de movimiento para obtener la respuesta de sitio.

\section{Fuentes Artificiales.}

Otra opción es el uso de fuentes artificiales. Estas pueden ser explosiones producto de pruebas militares, minería, construcción o bien impactos deliberados cuyo fin es el de sondear la 
estructura del suelo. Las fuentes artificiales son de especial interés en estudios de movimiento de suelo ya que el recorrido y la fuente de la onda son bien conocidos. Además, se puede controlar el tiempo, ubicación y magnitud antes del evento. Lo anterior hace eficiente el uso de los instrumentos de medición y recursos humanos.

Desafortunadamente es poco común contar con información proveniente de explosiones grandes. Ya sea porque en la actualidad no se hacen pruebas militares con bombas nucleares o porque las explosiones para extracción de recursos minerales no suelen hacerse cerca de centros urbanos, en donde es de interés el estudio de la respuesta del suelo. Por otro lado las fuentes de intensidad moderada, como lo son las utilizables en un ambiente urbano, provocan señales generalmente pobres para bajas frecuencias, que no pueden penetrar más allá de unas decenas de metros (Wathelet et al., 2005).

\section{Ruido sísmico ambiental.}

El ruido sísmico ambiental, también denominado microtremor, consiste en vibraciones debidas a fenómenos naturales y/o actividades humanas que se propagan por el suelo en forma de ondas elásticas. Como ejemplo de fuentes de ruido sísmico ambiental se pueden mencionar las olas de mar, el viento, el tráfico en las ciudades, el paso de maquinaria pesada etc. (Taga, 1993). El ruido sísmico ambiental se origina por diferentes fenómenos que pueden ser clasificados de acuerdo a la frecuencia. Las olas oceánicas golpeando las costas originan ruido en un rango de frecuencias que va de 0.05 a $0.1 \mathrm{~Hz}$, monzones y perturbaciones meteorológicas de gran escala en un rango de 0.1 a $0.25 \mathrm{~Hz}$, ciclones sobre los océanos de 0.3 a $1.0 \mathrm{~Hz}$, condiciones meteorológicas a escala local de 1.4 a $5.0 \mathrm{~Hz}$, tremor volcánico de $2.0 \mathrm{a}$ $10 \mathrm{~Hz}$ y por ultimo fuentes antrópicas de 1 a $100 \mathrm{~Hz}$ (Gutenberg, 1958).

El estudio de microtremor ha sido utilizado en Japón para determinar las frecuencias predominantes asociadas a estructuras de subsuelo desde 1966 (Kanai et al., 1966). Una de las ventajas de usar esta fuente de información es la facilidad con la que se hace el registro. El ruido sísmico ambiental tiene la ventaja de tener habitualmente un rango espectral amplio, comparado con una fuente artificial de pequeña intensidad (García-Jerez, 2010). Otra ventaja es que puede ser medido en cualquier momento y con instrumentación sencilla debido a que se genera de manera continua.

Un inconveniente de este tipo de movimiento es que puede no existir similitud entre el espectro registrado para terremotos y el espectro de microtremor en un mismo punto. Lo cual 
se debe a que las ondas registradas son de diferente tipo y tienen diferentes trayectorias de propagación (Udwadia y Trifunac, 1973).

Simulaciones numéricas han permitido concluir que la composición del ruido sísmico depende de dos factores principales. En primer lugar la posición de las fuentes, ya sean lejanas o cercanas y superficiales o profundas. En segundo lugar de la estructura del sitio, del contraste de impedancia entre la capa sedimentaria y el basamento y de sus frecuencias de resonancia (Bonnefoy-Claudey et al., 2006).

\subsection{Métodos de estimación de la respuesta de sitio.}

Para analizar y estimar la respuesta sísmica local se pueden utilizar tres tipos de métodos (Luzón et al., 2002), los basados en medidas instrumentales (métodos empíricos), los que utilizan registros de sismos pequeños como funciones de Green empíricas para simular acelerogramas de movimiento fuerte (métodos semiempíricos) y los que realizan simulaciones numéricas basadas en datos detallados de la estructura geológica local (métodos teóricos).

El primero consiste en medir directamente la amplificación del movimiento sísmico mediante técnicas empíricas que obtengan la función de transferencia del sitio con registros de sismos, explosiones o microtremores. El segundo método, inicialmente propuesto por Hartzell (1978), consiste en usar registros de sismos pequeños como funciones empíricas de Green para simular acelerogramas de movimiento fuerte que pueden servir para estimar el nivel del movimiento del suelo en una cuenca sedimentaria. El tercero consiste en caracterizar la respuesta dinámica del suelo conociendo de antemano las propiedades geofísicas de su estructura. Los parámetros que nos son útiles para este fin, en el caso de ambientes geológicos analizados unidimensionalmente (1D), son las velocidades de ondas $\mathrm{P}$ ○ $\mathrm{S}$, la densidad, la atenuación y la profundidad de las capas de suelo. Para obtener dichos parámetros una de las vías posibles es hacer uso del análisis de ondas superficiales.

\section{Técnicas empíricas para obtener la función de transferencia del sitio.}

Los métodos empíricos para obtener la función de transferencia del sitio se dividen en dos. El primero es el método de la estación de referencia o de la razón espectral con estación de referencia (método SBSR). Este método utiliza la información de una serie de estaciones, una de las cuales está ubicada sobre un afloramiento del lecho rocoso (estación de referencia) a poca distancia de donde se quiere analizar el efecto de sitio; las demás se ubican sobre los 
puntos a ser estudiados. Este método consiste en obtener la razón espectral entre las componentes de movimiento horizontal de las estaciones los sitios de interés y la estación de referencia. Usualmente la parte fuerte de un registro de terremoto, ondas $S$, es la que se usa para estimar la razón espectral relativa a la estación de referencia. Se busca que los registros de la estación de referencia sean representativos del lecho rocoso sobre el cual se ubica la estructura geológica que se desea caracterizar. Haciendo esto se acepta implícitamente que los efectos de sitio pueden ser caracterizados por modelos de incidencia vertical en una dirección. (Lermo y Chávez-García, 1993). En una primera aproximación, la relación SBSR, con una buena relación señal-ruido, debería mostrar las características de respuesta local porque los efectos de la fuente, el camino, y la instrumentación de medición quedan eliminados (Borcherdt, 1970). Dicha relación debe ser idéntica para desplazamiento, velocidad y aceleración.

El segundo es el método de la razón espectral usando una sola estación (H/V) o método HVSR, que puede usar registros de terremotos o de microtremores. Éste método fue primeramente desarrollado por Nakamura (1989) para analizar las ondas Rayleigh en registros de microtremor, razón por la cual esta técnica es comúnmente llamada razón de Nakamura. Nakamura consideraba que la amplificación espectral de una capa superficial sobre un semiespacio puede ser obtenida evaluando la razón espectral entre la componente horizontal y la componente vertical de registros de microtremor para un mismo sitio. La hipótesis en la que se sustenta la técnica es que la componente vertical del movimiento del suelo contiene más información de la fuente que la componente horizontal, por otro lado los efectos de la geología local son mayores en el movimiento del suelo horizontal que en el vertical.

\section{Técnicas de análisis de ondas superficiales para obtener la estructura de formaciones sedimentarias.}

La principal hipótesis que permite el uso del microtremor en estudios de caracterización de la respuesta dinámica del suelo, es que éste está constituido principalmente por ondas superficiales. En medios verticalmente heterogéneos, las ondas superficiales son dispersivas, lo cual controla la profundidad de penetración (Aki y Richards, 2002). Esta propiedad dispersiva puede ser usada para derivar la velocidad de onda S contra la profundidad de las capas de suelo usando un proceso de inversión (Wathelet et al., 2004). En ingeniera sísmica, la velocidad de onda $\mathrm{S}$ de la estructura de suelo es considerada un parámetro clave debido a su gran influencia en la amplificación del movimiento (Bard, 1994). 
Actualmente existen dos familias de métodos para la extracción de la curva de dispersión (Función frecuencia -velocidad) de un de suelo estratificado. Los métodos $f-k$, (frecuencia número de onda) y los métodos SPAC (Auto correlación espacial). La primera clase de métodos asumen que una única onda dominante se propaga por un arreglo de estaciones. Un simple proceso de sumatorias y cambio de fase, permite recuperar la velocidad y azimut aparente de la onda medida (Wathelet et al., 2005). El uso de ondas superficiales generadas por medios artificiales ofrece una ventaja a este tipo de métodos, pero como ya se ha visto antes, el contenido frecuencial de este tipo de ondas es una limitación de la profundidad alcanzable. En el caso de ondas viajando simultáneamente en varias direcciones, como es el caso del ruido sísmico ambiental, la suposición de señales no correlacionadas no puede ser satisfecha, lo cual origina estimas incorrectas en la velocidad (Goldstein y Archuleta, 1987).

Por otro lado, los métodos SPAC asumen que se cuenta con muestras de ruido sísmico ambiental en un arreglo de estaciones sobre una superficie libre y que se obtienen las funciones de correlación cruzada entre diferentes pares de estaciones, con la misma distancia entre ellas, muestreando diferentes orientaciones. En el caso de velocidades de fase con valores únicos por frecuencias, Aki (1957) demostró que las razones entre las funciones de correlación cruzada y las velocidades de fase tienen la forma de una función Bessel de orden cero cuyo argumento es dependiente de los valores de la curva de dispersión y de la apertura del arreglo de medición. Los métodos SPAC tienen la ventaja de contar con una distribución relativamente homogénea de las fuentes del campo de ondas, para de esta manera relacionar las razones de auto correlación con las velocidades de fase.

\subsection{Aplicabilidad y antecedentes de los métodos HVSR y SPAC.}

\section{Método de la razón espectral usando una sola estación de medición “HVSR”.}

El artículo original de Nakamura se ha convertido en uno de los más citados en sismología y hoy en día es usado prácticamente en todos los estudios de microzonificación. Esto es bastante curioso si se considera que los sismólogos aún no han llegado a un consenso sobre la teoría que sustenta al método (Sánchez- Sesma et al., 2011), y el debate continua enfocándose principalmente en la interpretación de la composición del ruido sísmico ambiental, ya sea que éste esté constituido por ondas superficiales o por ondas internas (Herak, 2008). 
Como ya se ha señalado, esta metodología fue ideada para usar microtremor en la estimación de la amplificación del movimiento horizontal de las capas superficiales durante terremotos. En principio el procedimiento presenta las ventajas de usar solo una estación de registro con tres componentes y de no necesitar la ocurrencia de un terremoto ya que el ruido sísmico ambiental es el que provee la información.

Muchos estudios han demostrado que la HVSR puede revelar, con cierta garantía, la frecuencia fundamental en un sitio. La capacidad de revelar dicha frecuencia aumenta si existe un gran contraste de impedancias entre el lecho de roca y la capa de suelo (Luzón et. al., 2001). Ohmachi et al., (1991) usaron la técnica de Nakamura con éxito en el área de San Francisco; Lermo (1992) hizo lo propio para cuatro sitios en la ciudad de México y obtuvo resultados que concuerdan con los obtenidos mediante razones espectrales estándar (SBSR) usando registros de terremotos. Además se ha demostrado que los resultados con esta técnica son bastante buenos cuando se puede hacer un modelo en 1D del suelo en el sitio (Chávez-García et al., 1999).

La segunda forma de usar esta técnica es con registros de terremotos. Aunque en el presente trabajo no se usará la técnica con acelerogramas de terremotos, resulta interesante revisar las razones y conclusiones de trabajos que sí lo han hecho. Lermo y Chávez-García (1993) explicaron cómo es que una técnica concebida para analizar ondas Rayleigh en microtremor puede aplicarse usando la parte intensa (ondas S) de un acelerograma siguiendo un modelo matemático sencillo. Para ilustrar sus razones argumentaron lo siguiente: En la ciudad de México, durante el terremoto de Michoacán de 1985, la amplificación espectral en suelos arcillosos blandos alcanzó valores de 50 en algunas frecuencias (Singh et al., 1988). A pesar de estos grandes efectos de sitio, la componente vertical tuvo las mismas características y amplitudes sin importar el tipo de suelo de la estación de medición, ya sea de arcilla o de roca ígnea (Campillo et al., 1989).

La técnica fue usada por Lermo y Chávez-García en tres lugares de México, usando también el método de la estación de referencia para comparar los resultados. En los tres casos estudiados el pico promedio de la razón espectral calculada con la componente vertical queda en los márgenes de una desviación estándar de la razón espectral usando la estación de referencia en roca. Se llegó al resultado de que el comportamiento promedio de las funciones de transferencia empíricas tiene mayor dependencia de los efectos de sitio que con la directividad de la fuente. En los demás casos se utilizó la técnica de Nakamura en la propia estación de 
referencia y se observó que el promedio de la función es plano y con amplificación unitaria. Los resultados muestran que la técnica de Nakamura entrega estimados robustos de frecuencia y amplitud en el modo fundamental. Los resultados muestran que la técnica de Nakamura proporciona estimaciones robustas de la frecuencia y la amplitud en el modo fundamental. Los resultados sugieren que si los efectos de sitio son producidos únicamente por la geología local, la misma hipótesis necesaria para las técnicas estándar de razón espectral, es posible estimar el periodo dominante y el nivel de amplificación local usando registros de una sola estación.

Al Yuncha y Luzón (2000) estudiaron los alcances que podría tener el método cuando las condiciones del sitio deben ser modeladas mediante geometrías trapezoidales, triangulares, semicirculares o semi elípticas. Usaron el Método Indirecto de Elementos de Frontera para estudiar la respuesta de las cuencas sedimentarias descritas. Compararon los resultados del HVSR con aquellos obtenidos mediante el método de la estación de referencia SBSR. Llegaron a la conclusión de que el HVSR no puede predecir con precisión los niveles de amplificación para cada periodo en las cuencas estudiadas, por lo tanto no provee el periodo dominante del sitio. Esto nos indica que únicamente estructuras geológicas unidimensionales pueden ser analizadas con el método.

\section{Método de Auto-correlación espacial modificada "SPAC".}

Una forma de estimar el efecto de sitio es a partir del conocimiento de la estructura de la cuenca. Dicha estructura está compuesta por la geometría de la interface entre la capa de suelo y la base de roca, además de por la naturaleza y espesor de los sedimentos. El método de auto-correlación espacial modificada (SPAC) fue propuesto por Aki en 1957 para revelar la naturaleza del ruido sísmico ambiental y las características del medio de propagación. Bettig et al. (2001) definen el SPAC como un método diseñado para estimar las curvas de dispersión de las ondas superficiales mediante análisis de correlación entre registros de ruido sísmico hechos en sitios cercanos entre sí, dichas curvas pueden ser usadas para caracterizar la estructura del medio de propagación. Para Chávez-García et al. (2005) la esencia del método SPAC, radica en que, cuando se tienen registros de estaciones sísmicas, espaciadas a distancias constantes y formando pares a lo largo de diferentes azimuts, es posible calcular una estima de la velocidad de fase de las ondas viajando por el arreglo, sin tener que considerar la dirección en la que se propagan las ondas presentes. Dado que el método está basado en una investigación 
estadística en el tiempo y el espacio, podemos asumir que la señal es un ruido estocástico estacionario en ambos dominios (Bettig et al., 2001).

El SPAC es un método que puede determinar de forma exitosa, en comparación con otros métodos (refracción, reflexión, registro de pozo), el perfil de velocidad de la estructura del suelo (Vázquez et al., 2011). También es apropiado para usarse en áreas urbanas, aunque el arreglo de los puntos de medición puede llegar a ser un problema precisamente por las limitaciones de espacio.

El método SPAC ha sido utilizado por Kagawa et al. (1999) en la zona circundante a Granada para determinar la estructura geológica con el objetivo de hacer estudios de microzonificación sísmica. Llegando así a determinar curvas de dispersión cuya inversión arroja resultados con profundidades de hasta 1910 metros, encontrando una velocidad de onda de cizalla de 2006 $\mathrm{m} / \mathrm{s}$ en sitios como el Zaidín. El método también ha sido utilizado por Navarro et al. (2010) en 13 espacios abiertos de la ciudad con arreglos pentagonales, determinando la Vs30 en cada zona para la clasificación del terreno de la ciudad. En algunos puntos de la ciudad se logró encontrar el basamento ingenieril (Vs>750 m/s) aunque no se ha logrado obtener información de capas más profundas de 50 metros debido a la limitación en cuanto al tamaño de los arreglos usados.

\subsection{Objetivos del trabajo.}

La ciudad de Granada se encuentra en un área de sismicidad moderada, lo que genera carencia de registros de movimiento fuerte de suelo. Dicha falta de datos hace casi imposible el uso de técnicas que necesiten acelerogramas de terremotos, como p.e el Método de la estación de referencia en roca (Borcherdt, 1970). Además de esto, se ha comprobado que la profundidad de las capas sedimentarias de suelo blando puede llegar a ser muy grande, hasta 200 metros en algunas partes de la Cuenca de Granada (Morales et al., 1993). Dicha profundidad hace que las técnicas que usan fuentes artificiales de movimiento no sean capaces de caracterizar por completo la estructura del sitio.

Con el fin de determinar los valores de los parámetros necesarios para caracterizar la respuesta dinámica del suelo en distintos puntos de la ciudad de Granada, en este trabajo se propone hacer uso de herramientas pasivas y de bajo costo que sean capaces de darnos información suficiente para determinar modelos realistas que reproduzcan las propiedades 
geofísicas del suelo en la ciudad. Para esto se pretende usar mediciones de ruido sísmico ambiental en diferentes puntos para así obtener, mediante los métodos HVSR y SPAC, modelos de suelo que reproduzcan los efectos de sitio de los puntos estudiados. Una vez que se tenga esta información se pretende hacer simulaciones de movimiento con registros en roca de terremotos reales (de zonas sismogenéticas similares a la que pertenece la ciudad de Granada) para obtener las características del movimiento esperable en cada sitio en el que se ha determinado el modelo de estructura superficial del terreno en términos de Vs.

\section{Objetivos específicos.}

Usando muestras de ruido sísmico ambiental medidas en distintos puntos de la ciudad de Granada se plantea llegar a los siguientes objetivos.

- Tomar muestras de microtremor suficientemente largas para tener curvas HVSR estables y encontrar el periodo predominante de puntos representativos de la ciudad de Granada.

- Calcular a partir de las muestras de ruido sísmico ambiental obtenidas con arrays curvas de dispersión y curvas HVSR. Usando en el caso de las curvas HVSR varios programas computacionales para comparar los distintos métodos de cálculo.

- Llevar a cabo una inversión conjunta de las curvas de dispersión y las curvas HVSR para obtener modelos de velocidad que reproduzcan las propiedades geofísicas del suelo en los puntos estudiados.

- Simular la respuesta dinámica del suelo en los sitios en donde se han hecho las inversiones conjuntas de los modelos de velocidad usando registros de terremotos reales de zonas con condiciones sísmicas similares a las de la ciudad de Granada.

- Calcular una serie de parámetros instrumentales del movimiento sísmico en superficie que caractericen la gravedad de la sacudida sísmica para los sismos simulados.

- Analizar los resultados y sacar conclusiones de las estructuras superficiales obtenidas y de la respuesta del suelo en la ciudad de Granada.

\subsection{Contenidos de la memoria.}

La presente memoria consta de seis capítulos que se detallan a continuación:

- En el capítulo 1 se hace una introducción general sobre el efecto de sitio y su importancia en el cálculo de la peligrosidad sísmica en un punto en concreto. Se 
presentan algunas ciudades en donde se han presentado importantes efectos de sitio a causa de terremotos históricos. Se hace una breve revisión de los métodos de estimación de la respuesta de sitio, con especial énfasis en el método de Nakamura y el método SPAC modificado.

- En el capítulo 2 se revisa la información geológica y geofísica de la zona de estudio, tanto las características generales de la Cuenca de Granada como lo referente al área urbana de la ciudad. Además, se presentan las características de las medidas en arreglos y en una sola estación de microtremor. Se incluye en éste capítulo un Test de Estacionaridad y estimación de la ventana óptima para el cálculo de las curvas HVSR.

- En el capítulo 3 se presenta el cálculo de las curvas de dispersión con el método SPAC modificado y las curvas HVSR. Se hace una descripción tanto del método SPAC como del método HVSR de microtremor. El cómputo de las curvas HVSR se hace con tres programas (HVSR Pwelch, HVSR MEM y Geopsy) con el fin de comparar y verificar los resultados. Al final del capítulo se comentan los resultados.

- En el capítulo 4 se obtienen modelos de suelo de los puntos en donde se cuenta con información SPAC Y HVSR haciendo uso del complemento "dinver" de Geopsy. Se explica el algoritmo de inversión del programa y se presentan y comentan sus resultados.

- En el capítulo 5 se hacen dos simulaciones con registros de terremotos reales de la respuesta dinámica del suelo en cada uno de los sitios en donde se ha hecho la inversión conjunta del modelo de velocidades. A partir de los acelerogramas propagados unidimensionalmente se calculan parámetros de movimiento sísmico que caractericen la gravedad de la sacudida sísmica. Los resultados de dichos parámetros de movimiento sísmico son comentados.

- En el capítulo 6 se recopilan las conclusiones generales. 


\section{Zona de estudio y Datos.}

En esta sección se revisa la información geológica y geofísica de la zona de estudio, tanto las características generales de la Cuenca de Granada como lo referente al área urbana de la ciudad. Además, se presentan las características de las medidas en arreglos y en una sola estación de microtremor, detallando los puntos de la ciudad en donde se tomaron las mediciones y el equipo utilizado. Se incluye en éste capítulo un Test de Estacionaridad y estimación de la ventana óptima para el cálculo de las curvas HVSR.

\subsection{Características geológicas y geofísicas de la zona de estudio.}

\section{Características generales de la zona de estudio.}

La zona de estudio es el área urbana de la ciudad de Granada, situada en una región cuya actividad sísmica es considerada como moderada. En esta ciudad se han registrado instrumentalmente un gran número de terremotos cercanos, todos ellos de magnitud moderada o baja $\left(M_{b}<5.5\right)$ y generalmente a profundidades de entre cinco y diecisiete kilómetros (Azañon et al., 2007). Ejemplo de dicha actividad son los terremotos de Santafé de 1911 o Albolote de 1956 (Figura 2.1). Lo anterior no implica que sismos de mayor magnitud como los de 1431 o 1806 no puedan volver a ocurrir (Feriche, 2012). Para dichos eventos de 1431 y 1806 se han estimado magnitudes momento de $7.5 \pm 0.3$ y $5.6 \pm 0.3$ respectivamente (Vidal et al., 2010), lo cual da muestra de la importancia de estudiar el comportamiento dinámico del suelo en la ciudad.

Desde un punto de vista geológico, la ciudad de Granada está situada en el borde nororiental de la Cuenca de Granada (Figura 2.2). Se trata de una cuenca neógena situada en el sector central de la Cordillera Bética que tiene una longitud máxima de unos 65 kilómetros de este a oeste y unos 40 kilómetros de norte a sur (Sáenz de Galeano et al., 2011). La cuenca Granada fue sometida a subsidencia continua entre el Mioceno Superior y el Cuaternario, a condiciones marinas en un periodo entre seis y once millones de años atrás y a sedimentación continental hasta hace aproximadamente medio millón de años (Navarro et al., 2010). Los sedimentos más antiguos son conglomerados, calcarenitas y margas depositadas en ambientes marinos durante el Tortoniense inferior, de nueve a diez millones de años atrás. La sedimentación marina continuó hasta el Tortoniense superior. La cuenca se elevó durante el Messiniense, de 
seis a siete millones de años atrás, aislándose así de otras circundantes. El relleno continental está constituido por conglomerados, limonitas, areniscas y calizas, depositados en la parte central de la cuenca por los ríos que drenan las montañas de los alrededores y también por ambientes lacustres (Navarro et al., 2010).

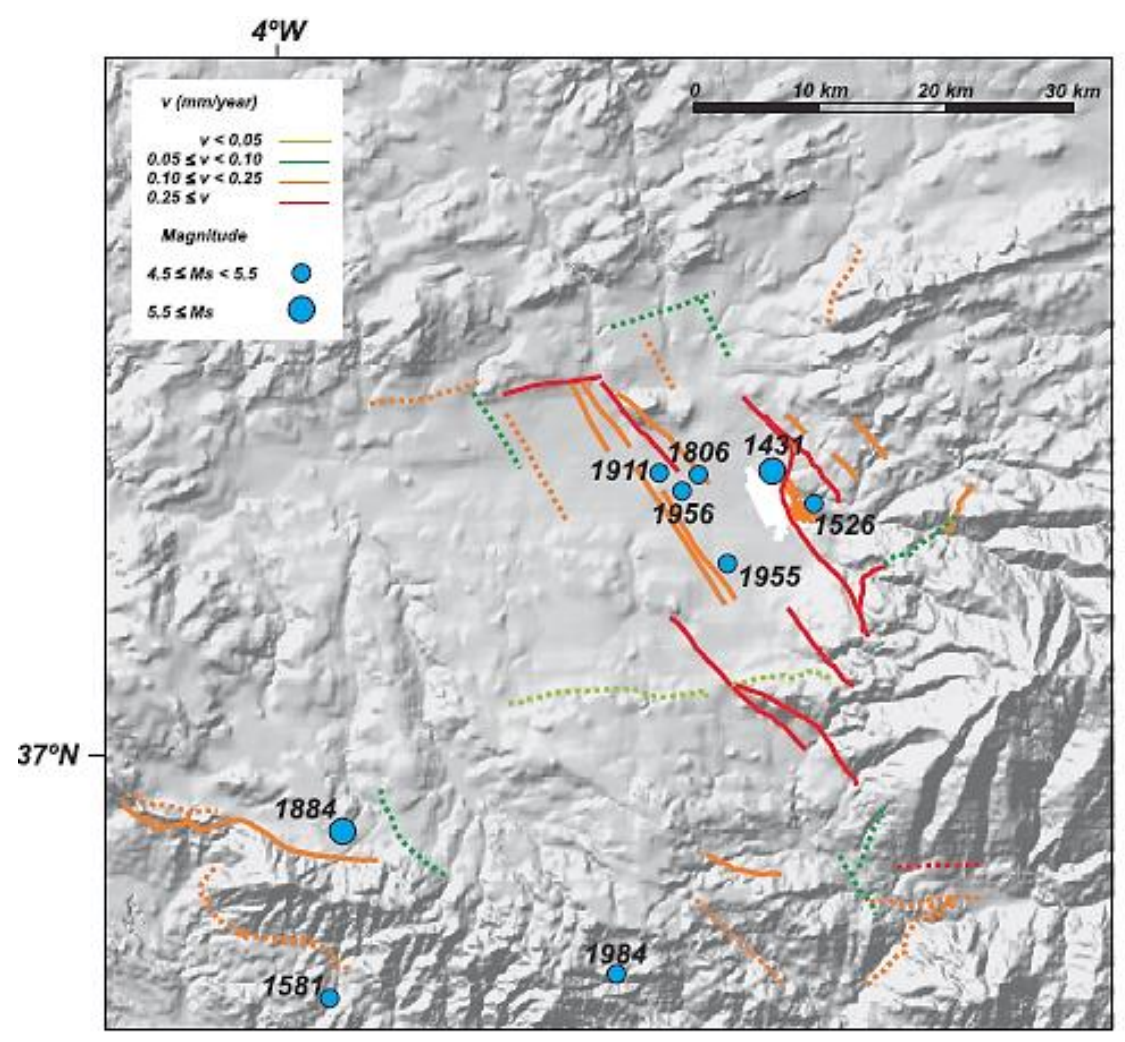

Figura 2.1 Localización de los terremotos más energéticos ocurridos los últimos 500 años en la cuenca de Granada y de las fallas activas y probablemente activas con su tasa de deslizamiento (Sanz de Galdeano et al., 2003). EI área urbana de Granada se muestra en blanco. Los epicentros y magnitudes de los terremotos históricos se asignaron a partir de datos macro sísmicos.

La Formación Alhambra, ubicada en la frontera noreste de la cuenca, emergió durante el Plioceno y Pleistoceno. Esta es constituida por conglomerados, arenas rojizas, limos y arcillas. Las gravas de esta formación, con una tamaño medio de $10 \mathrm{~cm}$, se formaron principalmente a partir de la erosión del basamento de la cuenca Granada. También se encuentran capas gruesas, de hasta un metro de espesor, de limo arcilloso (Feriche, 2012).

Los sedimentos más recientes son formados por abanicos aluviales creados en el Cuaternario que limitan la Sierra Alfaguara al norte y la Sierra Nevada al oeste. Estos abanicos aluviales están formados por partículas de grano grueso, a menudo cementadas, que van de arenas con gravas a grandes bloques de brechas. Pueden presentar intercalaciones finas, de limos arcillosos o paleosuelos rojos (Navarro et al., 2010). 


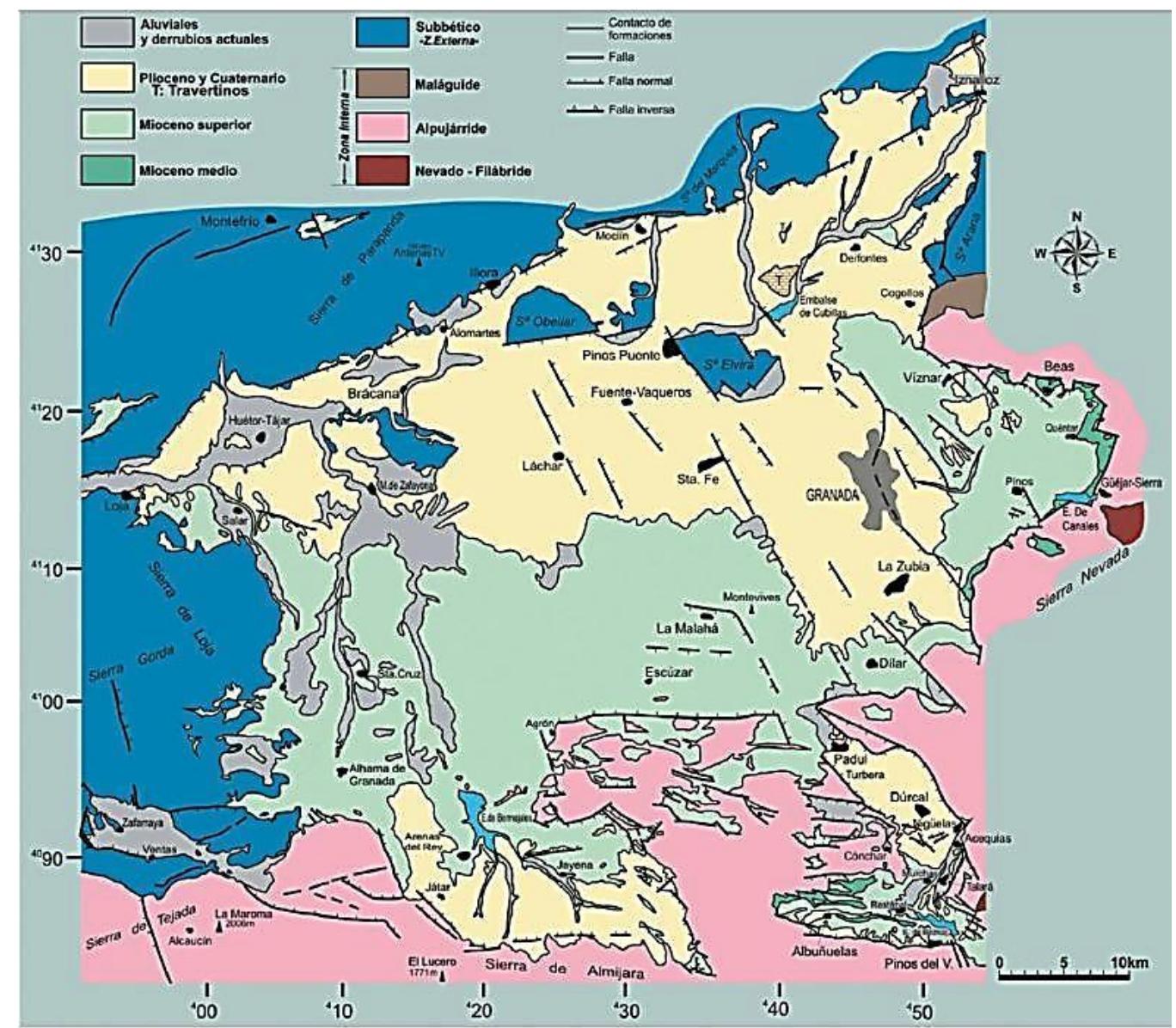

Figura 2.2 Esquema Geológico de la cuenca de Granada (Sanz de Galdeano, et al. 2001). En amarillo se muestran las áreas con materiales plio-cuaternarios, donde está ubicada la ciudad de Granada.

El relleno sedimentario de la cuenca de Granada es contemporáneo a la actividad de las fallas activas que la afectan, por lo que estas últimas han influido en la arquitectura estratigráfica de la cuenca. Estas fallas son por lo general normales, en su mayoría con una orientación Noroeste-Sureste y con inmersión hacia el Suroeste. En el norte de la ciudad, varios aluviales recientes se han formado en el bloque colgante de las fallas normales que cortan la formación Alhambra. (Navarro et al., 2010).

Los depósitos sedimentarios ubicados sobre Cubillas y Santa Fe llegan a tener hasta $3 \mathrm{~km}$ de espesor (Morales et al., 1993), con una importante presencia de depósitos superficiales del Holoceno de hasta 200 metros de espesor. La existencia de estos sedimentos recientes aunado a la presencia de agua en acuíferos situados cerca de la ciudad de Granada, muestran la importancia de determinar la estructura geológica superficial característica dentro de la zona urbana para la evaluación local de riesgo sísmico. 


\section{Características geotécnicas y geofísicas del área urbana.}

Para un mejor encuadre del área de estudio se han revisado diversos estudios que muestran las características geotécnicas y geológicas del área urbana de Granada

Valverde (2010) hizo un estudio de las características de los suelos con fines orientados a cimentación de edificaciones. El mapa de la Figura 2.3 muestra los valores medios de Vs del material portante (primeros metros) en función del número de golpes en ensayos de penetración estándar (SPT).

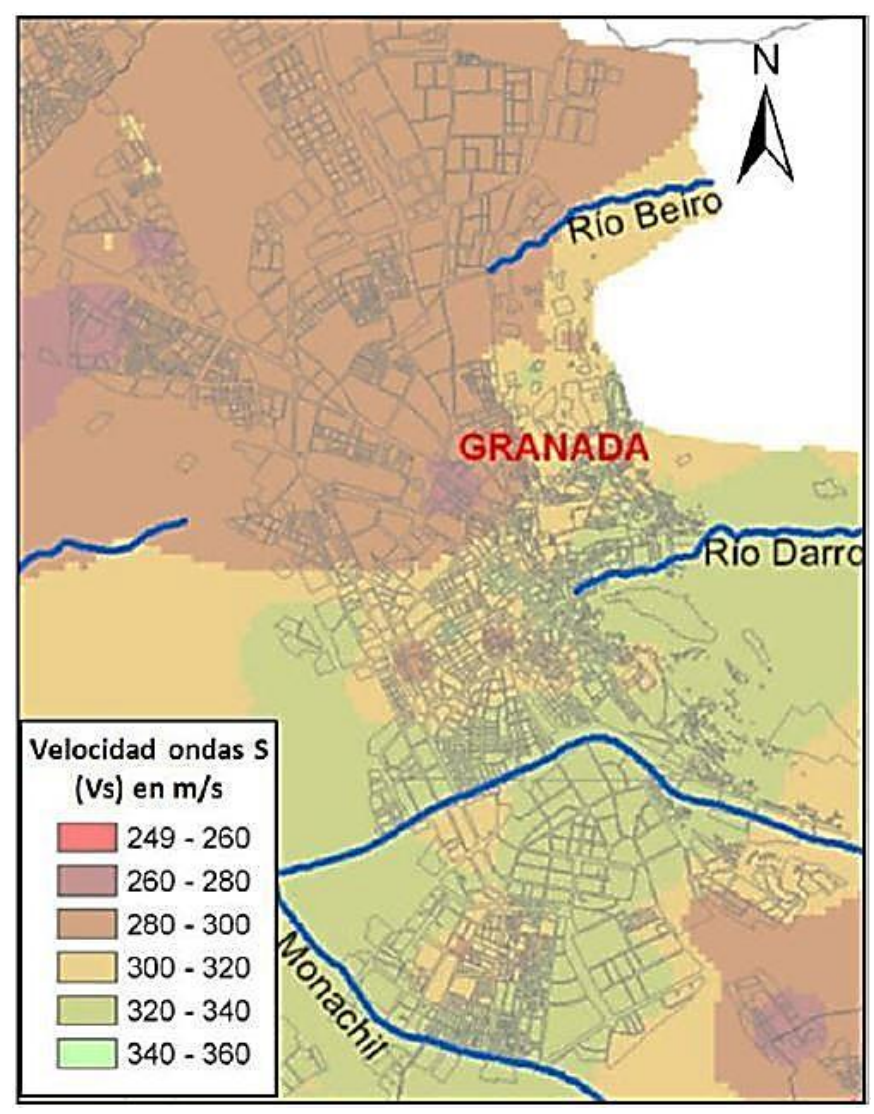

Figura 2.3 Representación gráfica del valor Vs medio en Granada (Valverde, 2010).

Hernández del Pozo (1998) obtuvo la distribución en la ciudad del espesor del material de relleno y de la profundidad del material competente a partir de un banco de datos geotécnicos, principalmente sondeos, y la cartografía de la ciudad. El espesor del material de relleno muestra irregularidad en la distribución de los rellenos de poco espesor, debido a la naturaleza antropológica de los mismos. Sin embargo, se aprecia una continuidad en los rellenos fluviales de considerable espesor en algunos tramos de los causes actuales de los ríos, respondiendo esta vez a fenómenos naturales de aportes sedimentarios de carácter fluvial 
En la Figura 2.4 se muestra el Plano de profundidad del material competente de la ciudad de Granada. Se define al material competente como aquel que es capaz de representar unos parámetros geotécnicos que verifiquen su aptitud para su uso en edificación (Feriche, 2012). Las isolíneas determinan la profundidad del nivel competente referida a la cota topográfica en el momento en el que se llevaron a cabo los sondeos. Dicha profundidad aumenta a medida que nos dirigimos hacia el norte noroeste de la ciudad. En áreas del este y del sur se aprecia poca profundidad del material competente.

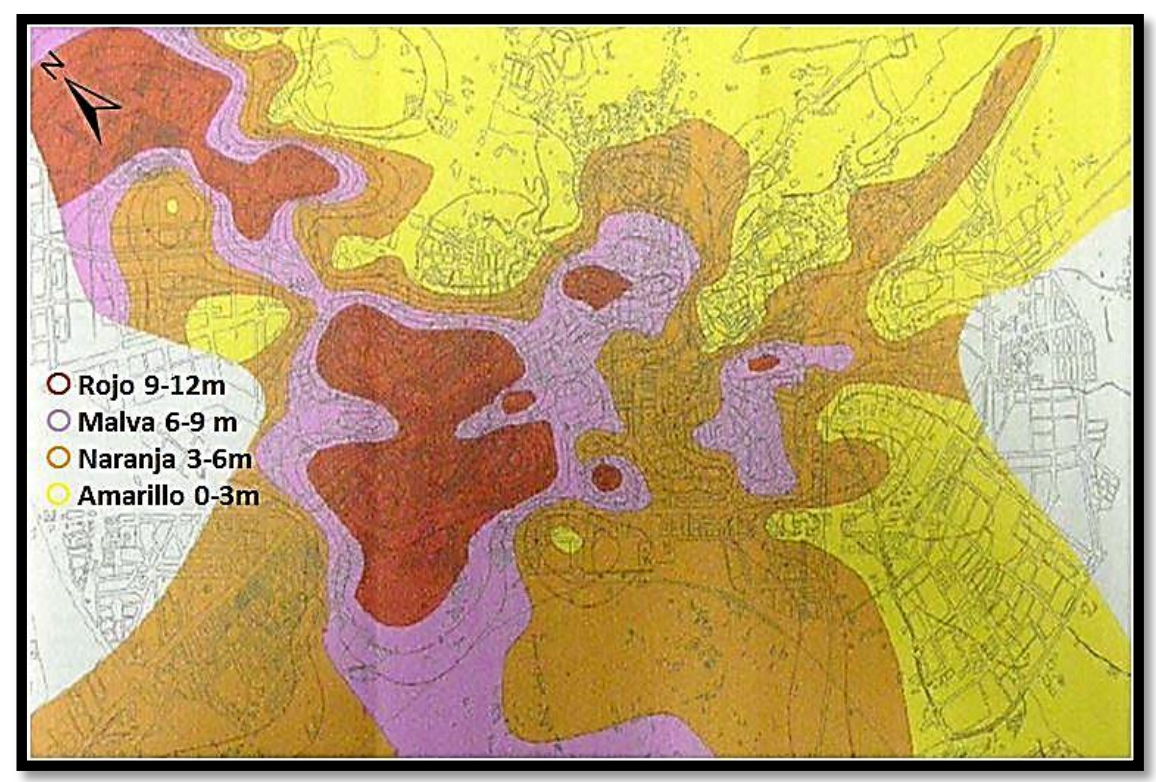

Figura 2.4 Plano de profundidad del material competente (Hernández del Pozo, 1998)

La microzonificación sísmica de la ciudad de Granada fue realizada por Cheddadi (2001) a partir de perfiles sísmicos de refracción y medidas de microtremor. Este trabajo se desarrolló con una metodología que permite su aplicación en áreas urbanas en regiones con sismicidad moderada, periodos de retorno largos y en donde no se dispone de datos de aceleración para aplicar técnicas directas. El estudio se hizo en dos partes, la primera con velocidades de ondas $\mathrm{S}$ (Vs) de las capas más superficiales (Figura 2.5), y la segunda con los periodos dominantes de suelo aplicando la técnica de Nakamura a microtremor medidas en una malla densa (Figura 2.6). 


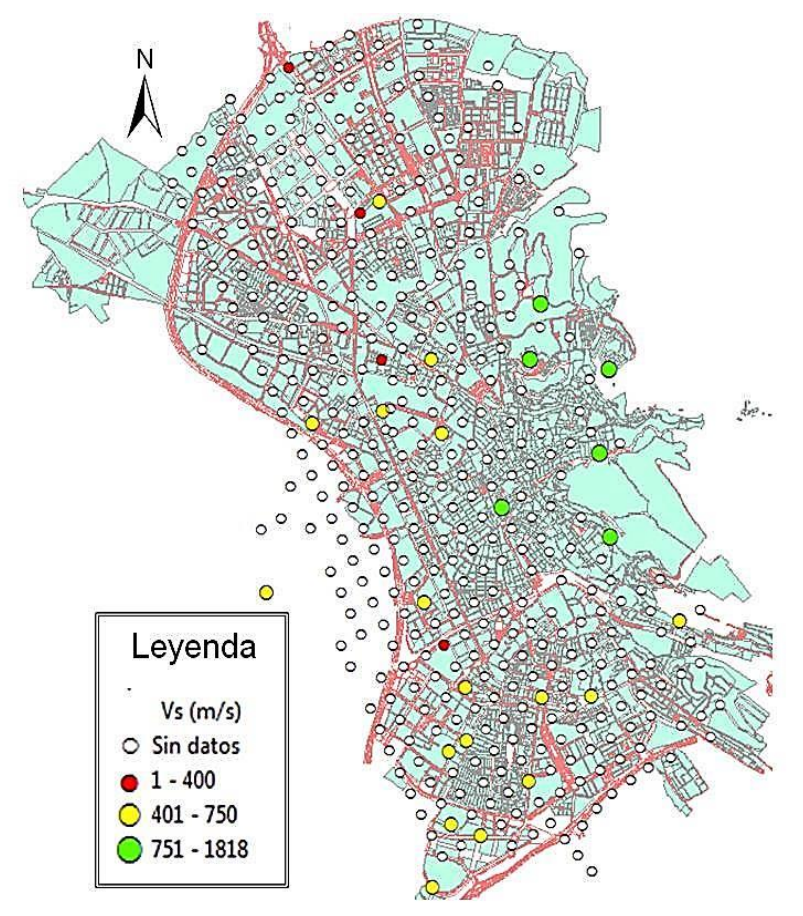

Figura 2.5 Microzonificación elaborada a partir de velocidades Vs por Cheddadi (2001)

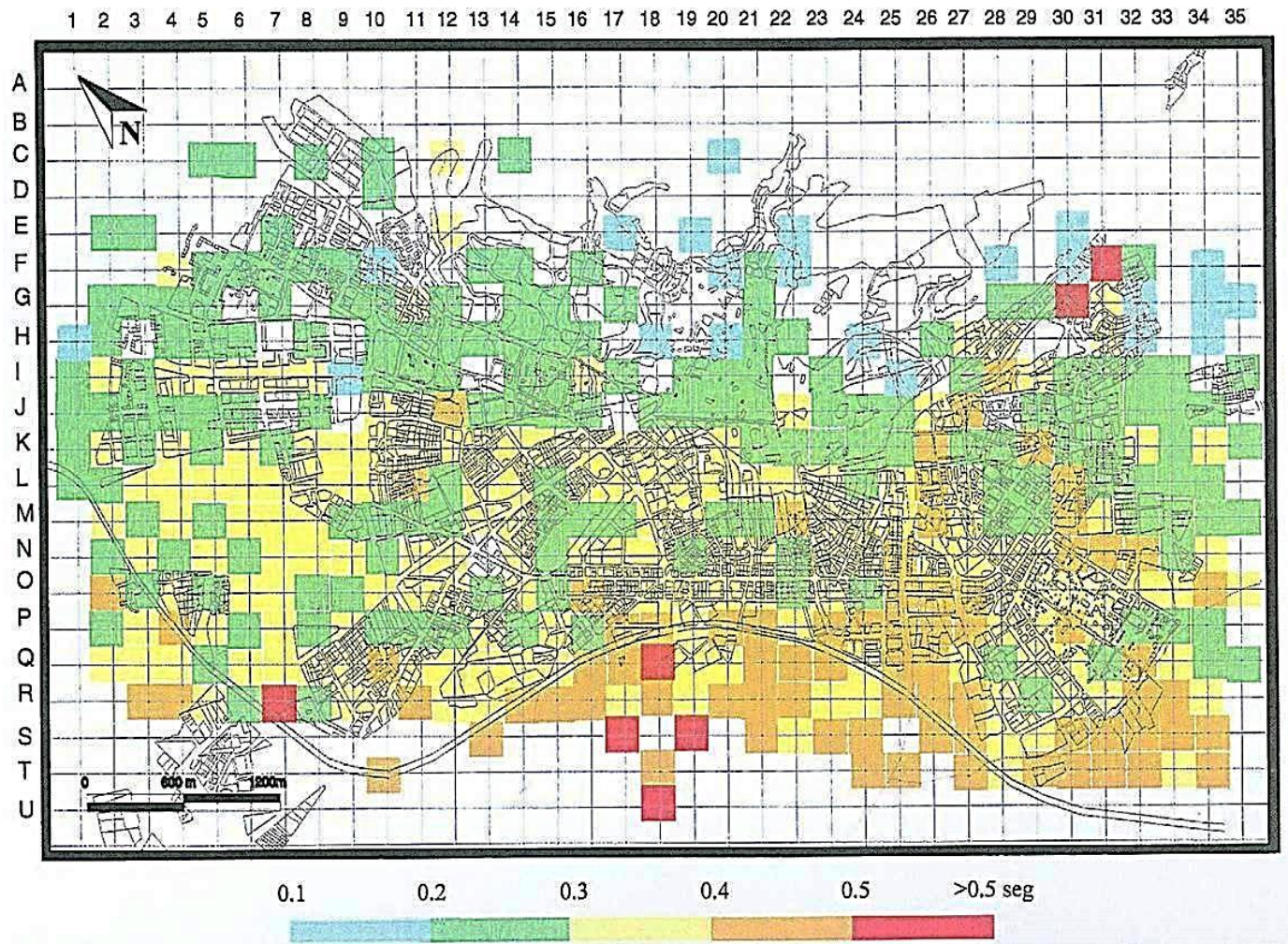

Figura 2.6 Microzonificación sísmica de Granada basada en períodos predominantes del suelo (Cheddadi, 2001). 


\subsection{Características de las medidas de microtremor en arreglos.}

Los datos que se usarán en el presente trabajo para el cálculo de la curvas de dispersión, que a su vez servirán para la inversión de los perfiles de velocidad, se han obtenido del trabajo de Navarro et al. (2011). Los puntos de la ciudad, 13 en total, que fueron estudiados se muestran en la Figura 2.7 y son:

- Aynadamar

- Zaidín

- Beiro

- Bola de Oro

- Fuentenueva

- Estadio de la Juventud

- Chana
- Náyades

- Parque de las Ciencias

- Granada 74

- Ferial

- Sanders

- Vega

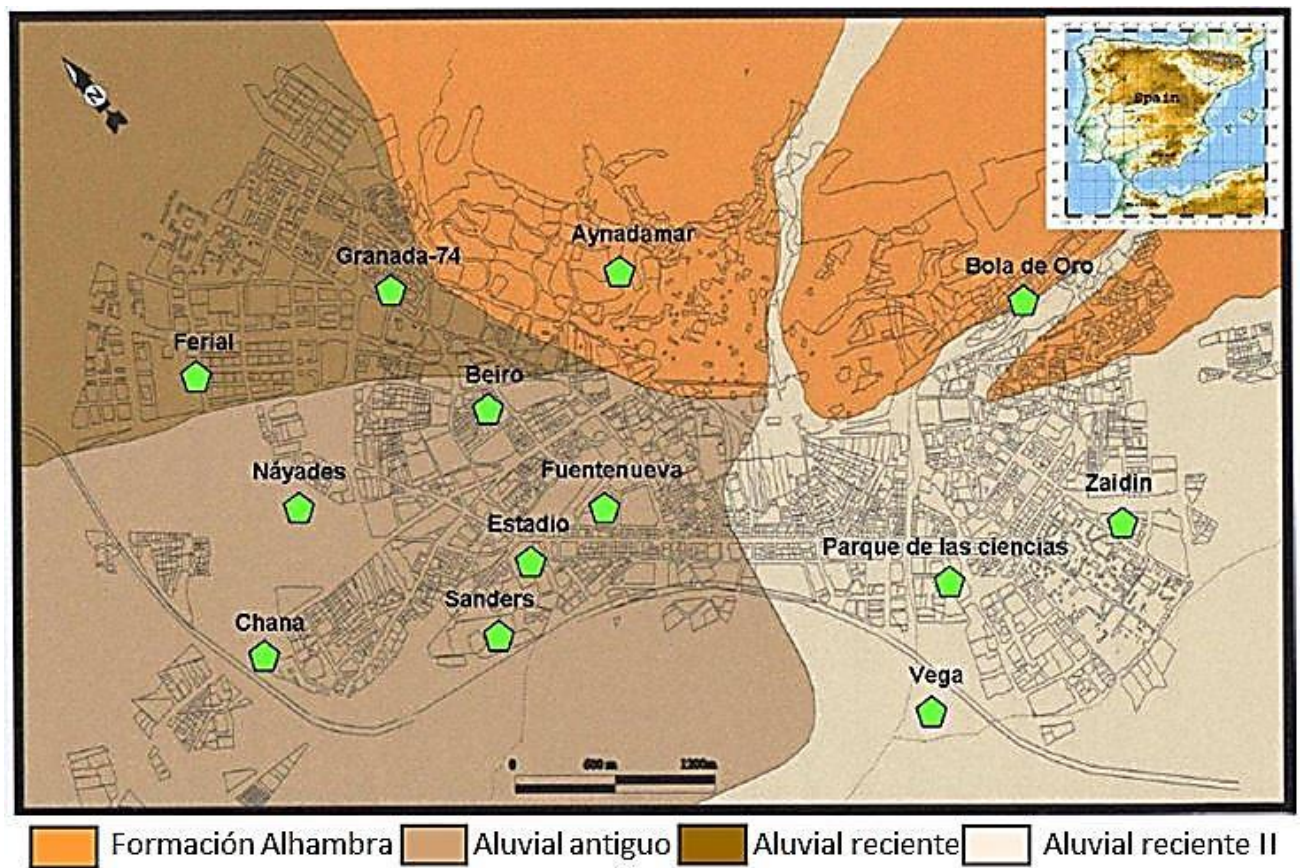

Figura 2.7 Localización de los puntos de toma de medidas de SPAC y principales unidades geológicas superficiales de la ciudad de Granada (Navarro et al, 2011)

Para la toma de datos se usó una red circular formada por seis sensores de componente vertical VSE-15D, cinco de los cuales se distribuyeron simétricamente a lo largo de una circunferencia y el último sensor se ubicó al centro de la misma (Figura 2.8). Los radios de cada uno de los sucesivos 3 o 4 dispositivos desplegados en cada sitio variaban de 3 a 30 metros dependiendo del espesor previsto de sedimentos a analizar y del espacio disponible en cada 
punto de medición. La grabación de datos duró 30 minutos en cada uno de los sucesivos dispositivos, con una señal muestreada a 200 muestras por segundo (Navarro et al. 2011)

Para el análisis de las señales, estas fueron divididas en intervalos de 20 segundos con un solapamiento del $80 \%$ y fueron transformadas al dominio de la frecuencia mediante la Transformada Discreta de Fourier. Los espectros complejos de las estaciones del perímetro se han promediado y dividido por el espectro de la estación central. Como resultado se obtiene el coeficiente de correlación SPAC $\rho(r, \omega)$, del cual se hablará en la sección 3.1 del presente trabajo.

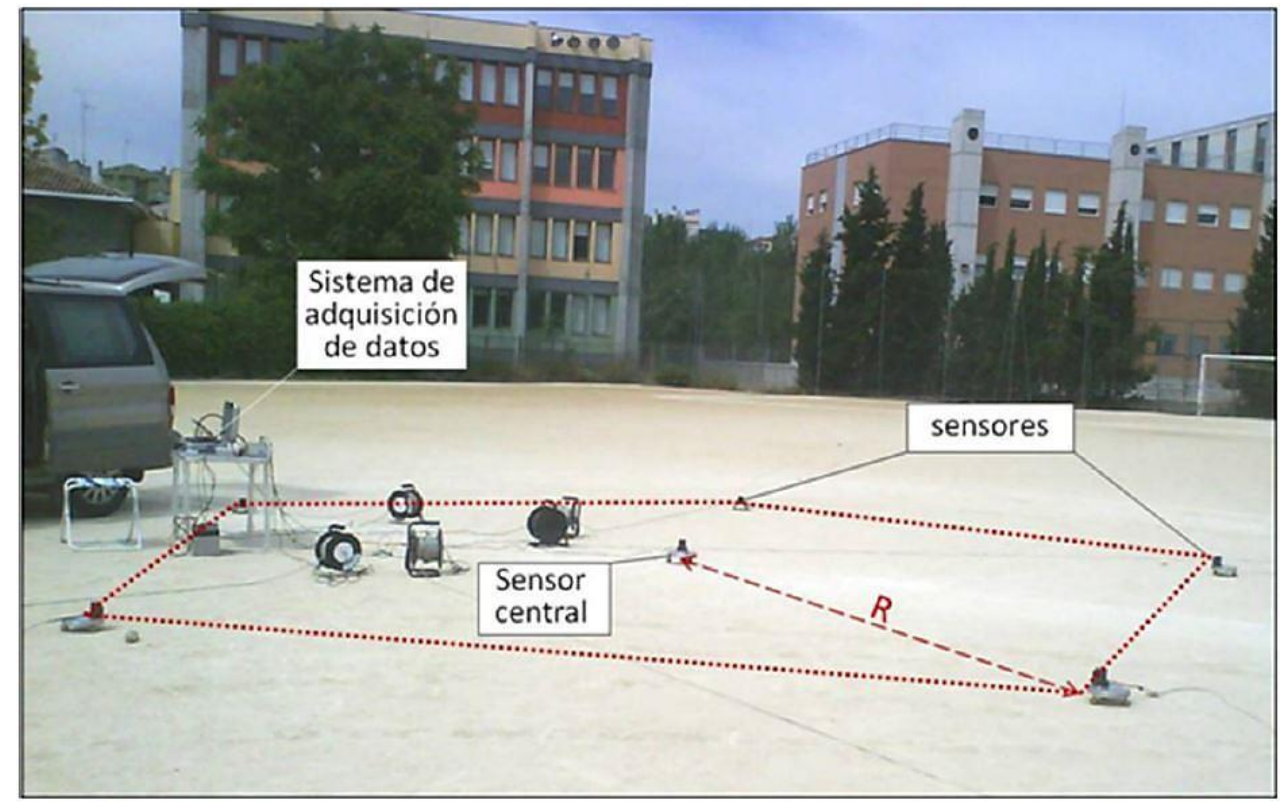

Figura 2.8 Despliegue del array en Fuentenueva que muestra los dispositivos de medición (Feriche, 2012)

\subsection{Características de las medidas de microtremor con una estación.}

Para analizar las características del periodo dominante del suelo en la ciudad de Granada se realizaron mediciones de ruido sísmico ambiental en los puntos que se citan a continuación y se muestran en la Figura 2.9:

- Centro deportivo Aynadamar.

- Parque Bola de Oro.

- Calle Aixa la Horra.

- Calle Atlanta.

- Calle Carmen Amaya.
- Escuela de Caminos, UGR.

- Calle Pampaneira (Estadio de la Juventud).

- Facultad de Filosofía y Letras, UGR.

- Campus Fuente nueva, UGR.

- Campo de futbol Granda 74. 
- Calle Náyades.

- Campo de los Mártires.

- Campo del Príncipe.

- Barrio de la Chana.

- Centro de Enfermería Virgen de las Nieves
- Calle la Malaha (Parque de las Ciencias).

- Beiro, Plaza de toros.

- Delegación Provincial de Salud y Bienestar Social.

- Facultad de Traducción e Interpretación, UGR.

- Zaidín.

Estos sitios fueron elegidos por diferentes motivos. En primer lugar se intentó repetir mediciones en los trece puntos de los cuales se tenía información SPAC de Navarro et al. (2011), los cuales son de gran importancia para obtener el perfil de Velocidades de onda S y espesores (Granada 74, Calle Náyades, Chana, Plaza de toros, Aynadamar, Bola de Oro, Fuentenueva, Estadio de la Juventud, Zaidín, Parque de las ciencias). Después, por la facilidad para realizar la medición, una serie de sitios pertenecientes a la Universidad de Granada (Escuela de Caminos, Escuela de Filosofía, Traductores). Por último se midieron algunos otros sitios en donde se buscaba algún resultado importante de amplificación espectral o como sitio de referencia en roca (Calle Aixa la Horra y Calle Náyades, Calle Atlanta, Calle Carmen Amaya, Campo de los Mártires, Campo del Príncipe, Enfermería y Sanidad).

\section{Características de los equipos utilizados.}

Para el cálculo la razón HVSR las muestras de microtremor se tomaron con una estación sísmica digital Taurus y un sensor sísmico triaxial Trillium Compact, ambos fabricados por Nanometrics. En la Figura 2.10 se muestran el sistema de adquisición y el sensor triaxial utilizados. El sensor triaxial tiene una respuesta uniforme de $20 \mathrm{~s}$ a $100 \mathrm{~Hz}$. Los ficheros de salida del digitalizador tienen formato MINISEED y se han hecho con frecuencia de muestreo de 100 muestras por segundo. Los ficheros de salida fueron procesados con el programa SEISAN para juntar los tres canales en un solo fichero de formato MSEED. En este mismo proceso fueron filtrados en un rango entre $0.2 \mathrm{~Hz}$. y $20 \mathrm{~Hz}$.

\section{Test de Estacionaridad y estimación de la ventana óptima.}

Para conocer cuál es la duración de registro para la que se estabiliza la razón espectral HVSR, se realizó un estudio estacionariedad y de elección de la Ventana Optima. El test de estacionariedad permite conocer si la estimación del periodo dominante en un sitio no depende del horario en el que se tomen las muestras. La ventana óptima es aquella que tiene 
una duración suficiente para proporcionar una estimación estable del periodo dominante en el sitio.

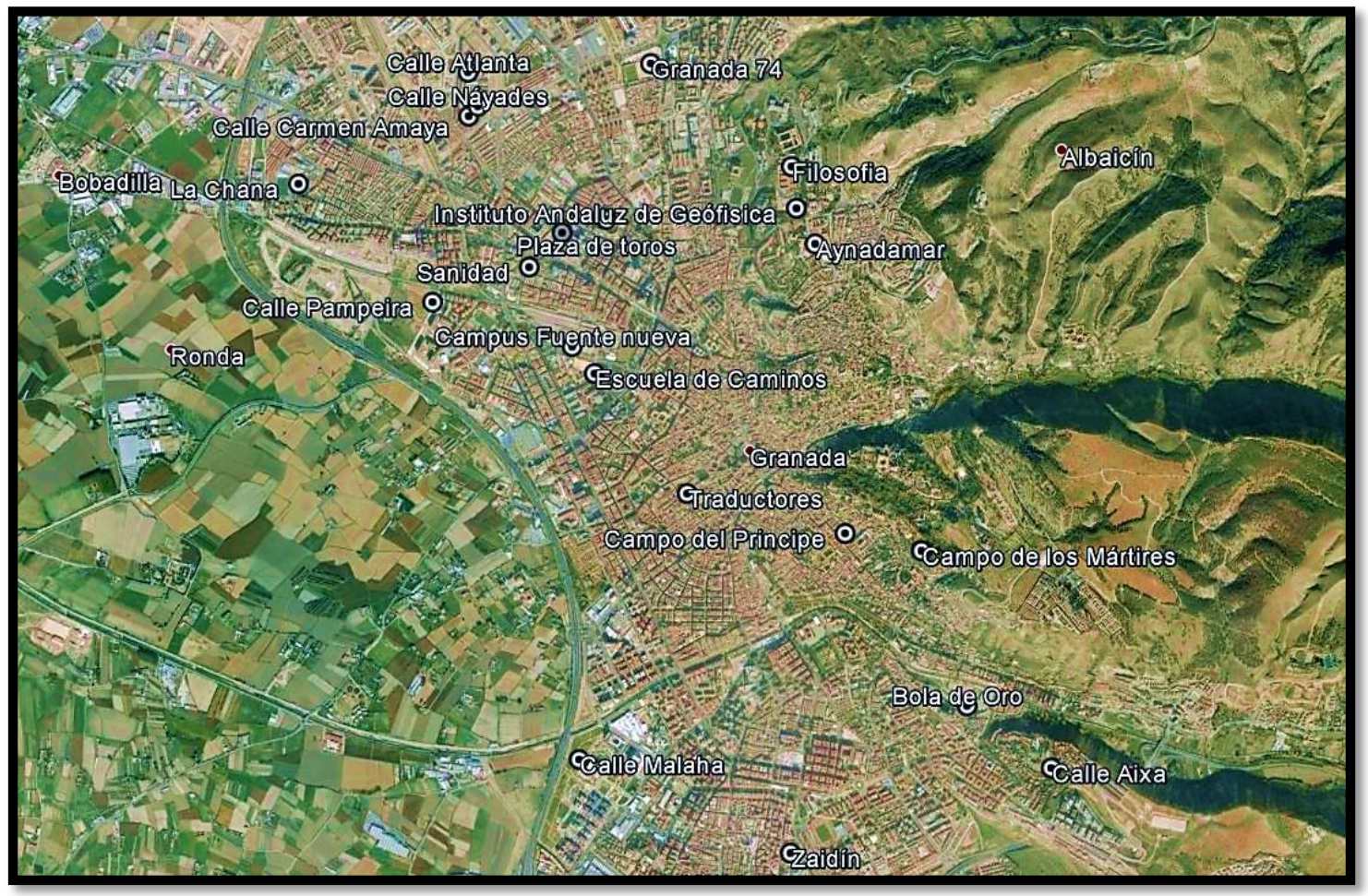

Figura 2.9 Mapa de la Ciudad de Granada con los sitios de las medidas de microtremor para usar la técnica HVSR.
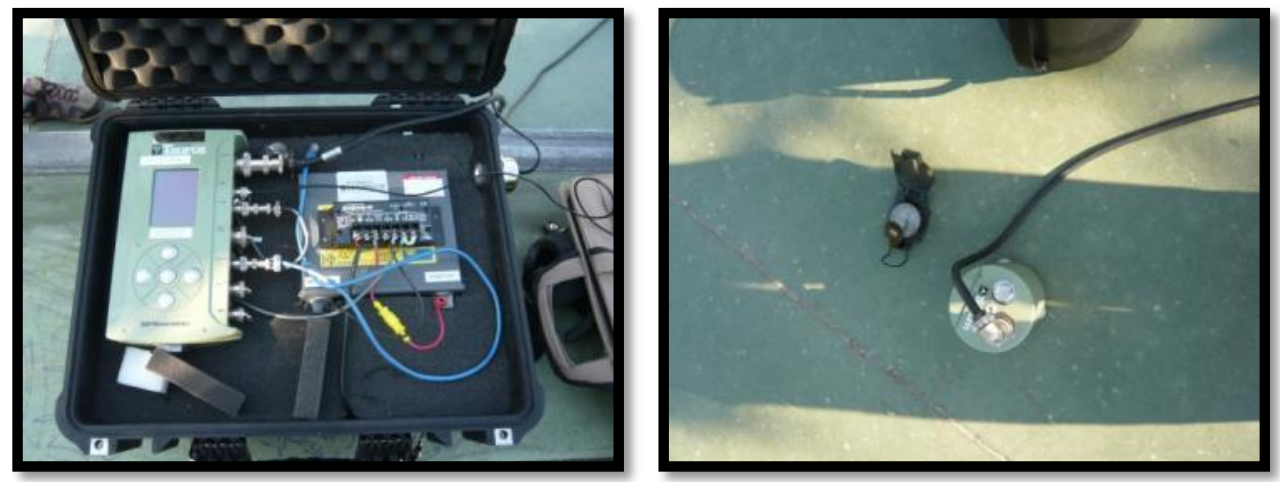

Figura 2.10 Estación sísmica y sensor sísmico Taurus.

Pa realizar dichos test, se tomaron mediciones en continuo en dos sitios: en la Calle Aixa la Horra y en la Calle Náyades, de tal forma que se viera la variación en el tiempo de la razón H/V y que se pudiera hacer una comparación entre las razones espectrales $\mathrm{H} / \mathrm{V}$ para el mismo sitio pero obtenidas con diferentes tiempos de medición o ventanas de registro. Cabe mencionar que en estos sitios se tenía la libertad de dejar trabajando el sismógrafo en continuo durante 
un lapso de 24 horas. Estos sitios tenían características de ruido y de estructura subsuperficial diferentes; el primero tenia las fuentes de ruido más alejadas que el segundo y una estructura del terreno más dura. El segundo sitio tenía una fuente de ruido espúrea cercana y la estructura del subsuelo es relativamente blanda. De esta manera podemos apreciar el punto en el cual dejan de existir múltiples picos en la razón espectral debidos a otras fuentes de excitación espúreas cercanas que son dominantes en el ruido y en la razón H/V pero que no se corresponden con las características geológicas locales.

Estas fuentes de excitación indeseables pueden ser causadas por el tráfico cercano de automóviles y personas, vibraciones en sitios de construcción cercanos, ráfagas de viento moviendo el alumbrado público, etc. Dichas fuentes indeseables pueden inyectar energía al suelo en una frecuencia que no llega a ser atenuada en el camino al sensor sísmico, lo cual genera picos dentro de la razón espectral que no son característicos del sitio sino de la fuente generadora, pero que conforme pasa el tiempo, y siempre y cuando sean transitorios, desaparecen o se atenúan bajando de amplitud en las frecuencias que ellos generan.

En el sitio de la Calle Náyades se puede esperar que el suelo atenúe de forma importante las vibraciones cercanas debidas al tráfico rodado y existencia de saltos en el pavimento. Navarro et al. (2011) obtuvieron un valor de Vs30 de 294 m/s para este sitio. En contraposición, el sitio de la calle Aixa la Horra está ubicado sobre la Formación Alhambra y por lo tanto se espera una menor atenuación.

La curvas de razón $\mathrm{H} / \mathrm{V}$ fueron obtenidas con el programa Geopsy (que se describe más adelante, dentro del apartado 3.2). Como se puede apreciar en la Figura 2.11 el sitio de la Calle Aixa la Horra presenta una buena estabilidad y estacionariedad para ventanas de 90 minutos, mientras que el sitio de la Calle Náyades lo hace para ventanas de 60 minutos (Figura 2.12). Este resultado es lógico por la razón antes dicha, el sitio sobre suelo blando atenúa de mejor forma las fuentes cercanas de excitación indeseable. Es destacable la aparición de niveles de ruido altos en el sitio de la Calle Aixa la Horra, en el rango de frecuencias de 4.0 a $5.0 \mathrm{~Hz}$ en horas nocturnas, debido probablemente a fuentes cercanas 


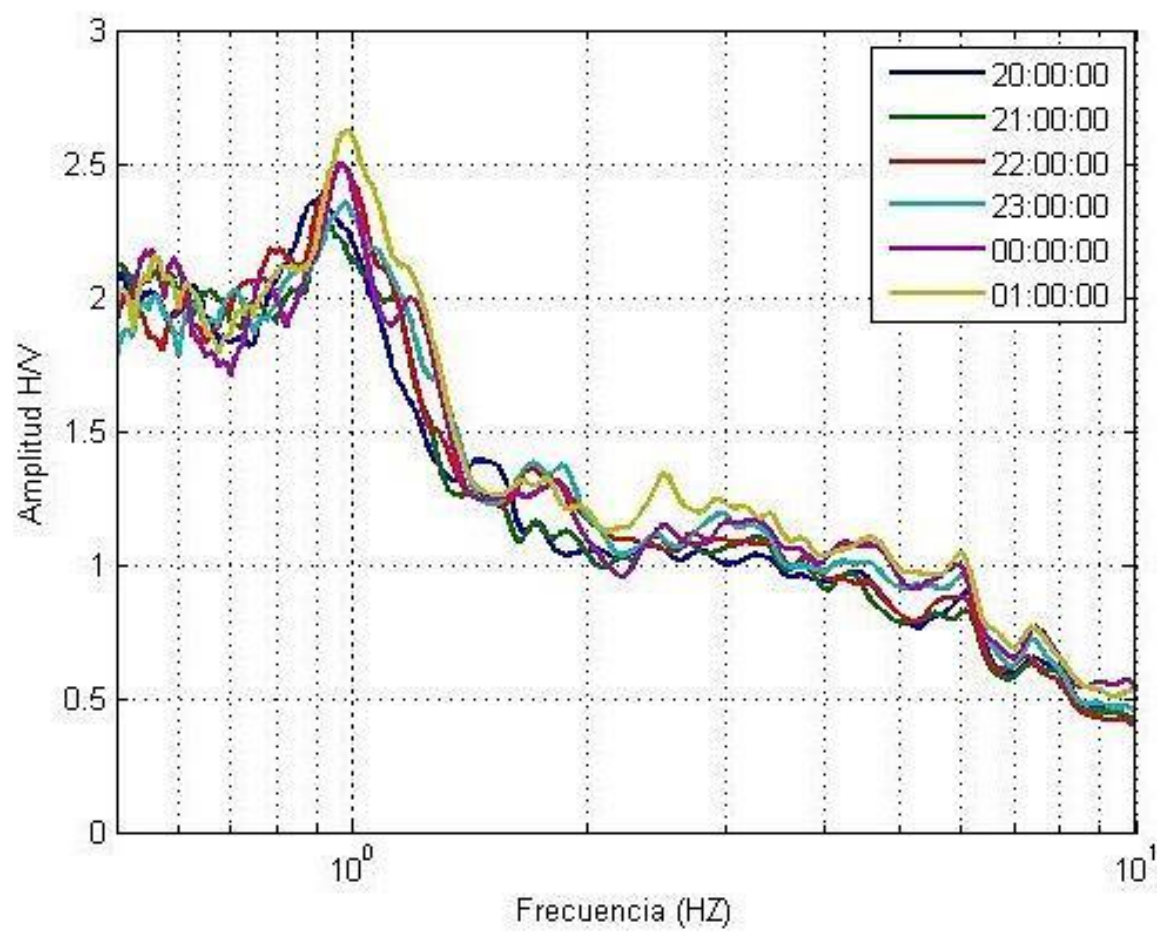

Figura 2.11 Estacionaridad de la respuesta de la razón HVSR en 60 minutos para el sitio de la Calle Náyades. Las gráficas se han calculado con el programa Geopsy.

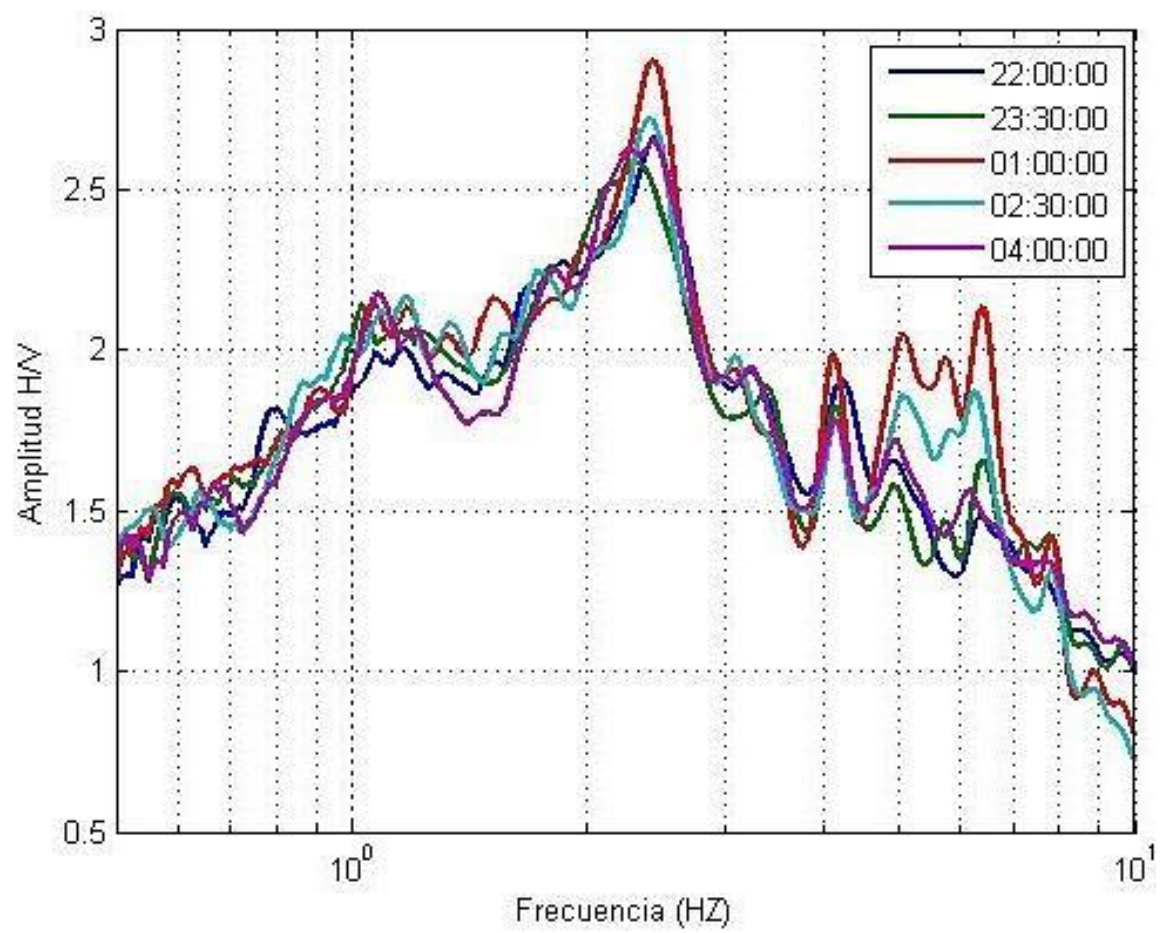

Figura 2.12 Estacionaridad de la respuesta de la razón espectral HVSR en 90 minutos para el sitio de la Calle Aixa la Horra. Las gráficas se han calculado con el programa Geopsy. 


\section{Cálculo de las curvas de dispersión y curvas HVSR.}

Se presenta el cálculo de las curvas de dispersión con el método SPAC modificado y las curvas HVSR. Se hace una descripción tanto del método SPAC como del método HVSR de microtremor. El cómputo de las curvas HVSR se hace con tres programas (HVSR Pwelch, HVSR MEM y Geopsy) con el fin de comparar y verificar los resultados. El funcionamiento de los programas también es descrito en la presente sección. Al final del capítulo se comentan los resultados obtenidos.

\subsection{Método de Auto correlación espacial modificada SPAC.}

\section{Descripción del método de SPAC modificado.}

El método puede describirse de la siguiente forma (Chávez-García et al., 2005). Si se cuenta con muestras de ruido sísmico ambiental en un arreglo de estaciones sobre una superficie libre; se obtienen las funciones de correlación cruzada entre diferentes pares de estaciones, con la misma distancia entre ellas, muestreando diferentes orientaciones en la superficie libre; y además se cumplen las siguientes dos hipótesis:

1) la vibración ambiental es estacionaria tanto en el tiempo como en el espacio,

2) el campo de ondas consiste en ondas dispersivas propagándose en toda la superficie.

Aki (1957) demostró que la razón entre el promedio de esas diferentes funciones de correlación cruzada y la función de auto-correlación en la estación de referencia (definida por Aki como el coeficiente de correlación), toma la forma de una función Bessel de primera clase y orden cero. En el argumento de la función de Bessel aparece la distancia fija de intersección, la frecuencia, y la velocidad de fase de las ondas propagadas. De este modo es posible obtener una curva de dispersión de la velocidad de fase usando registros filtrados en una serie de bandas de frecuencia estrechas

Aki (1957) considera un campo de ondas formado por la superposición de varias ondas planas no polarizadas propagándose en varias direcciones del plano horizontal, todas ellas con la misma velocidad de fase constante. El movimiento del suelo en los puntos $(x, y)$ y $(x+\xi, y+$ $\eta$ de la superficie será $u(x, y, t)$ y $u(x+\xi, y+\eta, t)$. La función de auto correlación espacial (Aki, 1957) es: 


$$
\phi(\xi, \eta, t)=\overline{u(x, y, t) u(x+\xi, y+\eta, t)}
$$

En donde $\overline{u(t)}$ es el valor medio de $u(t)$ en el dominio del tiempo. Asumiendo que el campo de ondas está compuesto por ondas estocásticas estacionarias, y reemplazando $(\xi, \eta)$ por una coordenada polar, Aki (1957) demostró que el promedio azimutal de la función de auto correlación espacial puede escribirse como:

$$
\bar{\phi}(r)=\frac{1}{2 \pi} \int \phi(r, \psi) d \psi
$$

En donde $r$ y $\psi$ son las coordenadas polares definidas por:

$$
\xi=r \cos (\psi) ; \eta=r \operatorname{sen}(\psi)
$$

Aki (1957) demostró que el promedio azimutal de la función de auto correlación $\bar{\phi}(r)$ y la densidad espectral de potencia $\Phi(\omega)$ del campo de ondas $u$, donde $(\omega)$ es la frecuencia angular, se relacionan como:

$$
\bar{\phi}(r)=\frac{1}{\pi} \int \Phi(\omega) J_{0} \frac{\omega}{c} r d \psi
$$

En donde $J_{0}$ es la función "Bessel" de orden cero. El argumento de la función Bessel puede escribirse también como:

$$
\frac{\omega}{c} r=k r=\frac{2 \pi r}{\lambda}
$$

En donde $k$ es el número de onda, y $\lambda$ es la longitud de onda. La ecuación anterior también se aplica al caso de ondas dispersivas como se muestra en Aki (1957). Se necesita substituir $c(\omega)$ por $c$. Se considera ahora que se aplica un filtro paso bandas a las señales. La densidad espectral se convierte en: 


$$
\bar{\Phi}(\omega)=P\left(\omega_{0}\right) \delta\left(\omega-\omega_{0}\right)
$$

en donde $P\left(\omega_{0}\right)$ es la densidad espectral de potencia en la frecuencia $\left(\omega_{0}\right)$ y $\delta(\omega)$ es la función "Dirac". En este caso, el promedio azimutal de la función de correlación espacial (Aki, 1957) puede escribirse como:

$$
\bar{\phi}(r) \equiv \bar{\phi}\left(r, \omega_{0}\right)=P\left(\omega_{0}\right) J_{0} \frac{\omega_{0}}{c\left(\omega_{0}\right)} r
$$

De acuerdo a Aki (1957), el coeficiente de auto correlación se definido como:

$$
\rho\left(r, \omega_{0}\right)=\frac{\phi\left(r, \psi, \omega_{0}\right)}{\phi\left(0, \psi, \omega_{0}\right)}
$$

Como $P\left(\omega_{0}\right)$ no depende de la posición, el promedio azimutal del coeficiente de auto correlación espacial puede escribirse como:

$$
\rho\left(r, \omega_{0}\right)=J_{0}\left(\frac{\omega_{0}}{c\left(\omega_{0}\right)}\right) r
$$

La derivación detallada se encuentra en Aki (1957) y en Chávez-García et al. (2005). El coeficiente SPAC $\rho(r, \omega)$, se obtiene en el dominio de la frecuencia usando la transformada de Fourier de los microtremores observados (Vázquez et al., 2011):

$$
\rho(r, \omega)=\frac{1}{2 \pi} \int_{0}^{2 \pi} \frac{R_{e} S_{c x}(\omega, r, \theta)}{\sqrt{S_{c}(\omega) \cdot S_{x}(\omega, r, \theta)}} d \theta
$$

En donde $R_{e}\left(\right.$ ) es la parte real del argumento complejo, $S_{c}(\omega)$ y $S_{x}(\omega, r, \theta)$ son las densidades espectrales de potencia de los microtremores en los sitios $c$ y $x$ respectivamente, $y$ $S_{c x}(\omega, r, \theta)$ es el espectro cruzado entre el movimiento de suelo de los dos sitios.

Los coeficientes SPAC deben ser obtenidos promediando la función de coherencia normalizada definida como el co-espectro entre los puntos $C$ y $X$ en la dirección $\theta$. 
De los coeficientes SPAC $\rho(r, \omega)$ la velocidad de fase es obtenida para cada frecuencia del argumento de la función de Bessel en la ecuación ( 3.9 ), en donde $c(f)$ es la velocidad de fase en la frecuencia $f$. El valor óptimo de $c(f)$ se obtiene usando una búsqueda de red (Vázquez et al., 2011).

\section{Cálculo de las curvas de dispersión con el método SPAC.}

A continuación se presentan las curvas de dispersión de los 10 sitios en los cuales se cuenta con información tanto de SPAC como de HVSR.

- Aynadamar

- Zaidín

- Beiro

- Bola de Oro

- Fuentenueva
- Estadio de la Juventud

- Chana

- Náyades

- Parque de las Ciencias

- Granada 74

Para obtener las curvas de dispersión los espectros complejos de las estaciones del perímetro de los arreglos se han promediado y dividido por el espectro de la estación central correspondiente. Como resultado se obtiene el coeficiente de correlación SPAC $\rho(r, \omega)$, ecuación ( 3.10$)$. Los coeficientes SPAC $\rho(r, \omega)$ y la velocidad de fase de ondas Rayleigh se relaciona mediante la ecuación ( 3.9 ). Una vez que se ha determinado el coeficiente de correlación, se calcularon las curvas de dispersión para diferentes rangos de longitud de onda en función del radio utilizado en cada uno de los dispositivos medidos en el arreglo. Después se combinaron estas para obtener una única curva de dispersión para cada sitio estudiado.

Con la metodología descrita se obtuvieron las curvas de dispersión en los diez sitios de Granada en los que se desplegaron los arrays que se muestran en la Figura 3.1. Las frecuencias de las curvas obtenidas varían entre 2.5 y $33.5 \mathrm{~Hz}$ y los valores de velocidad de fase van de 228 a $708 \mathrm{~m} / \mathrm{s}$. Se observó una buena correlación entre las formas de la curva de dispersión y las condiciones geológicas de cada sitio, y se pudieron detectar las variaciones laterales entre formaciones geológicas adyacentes. 

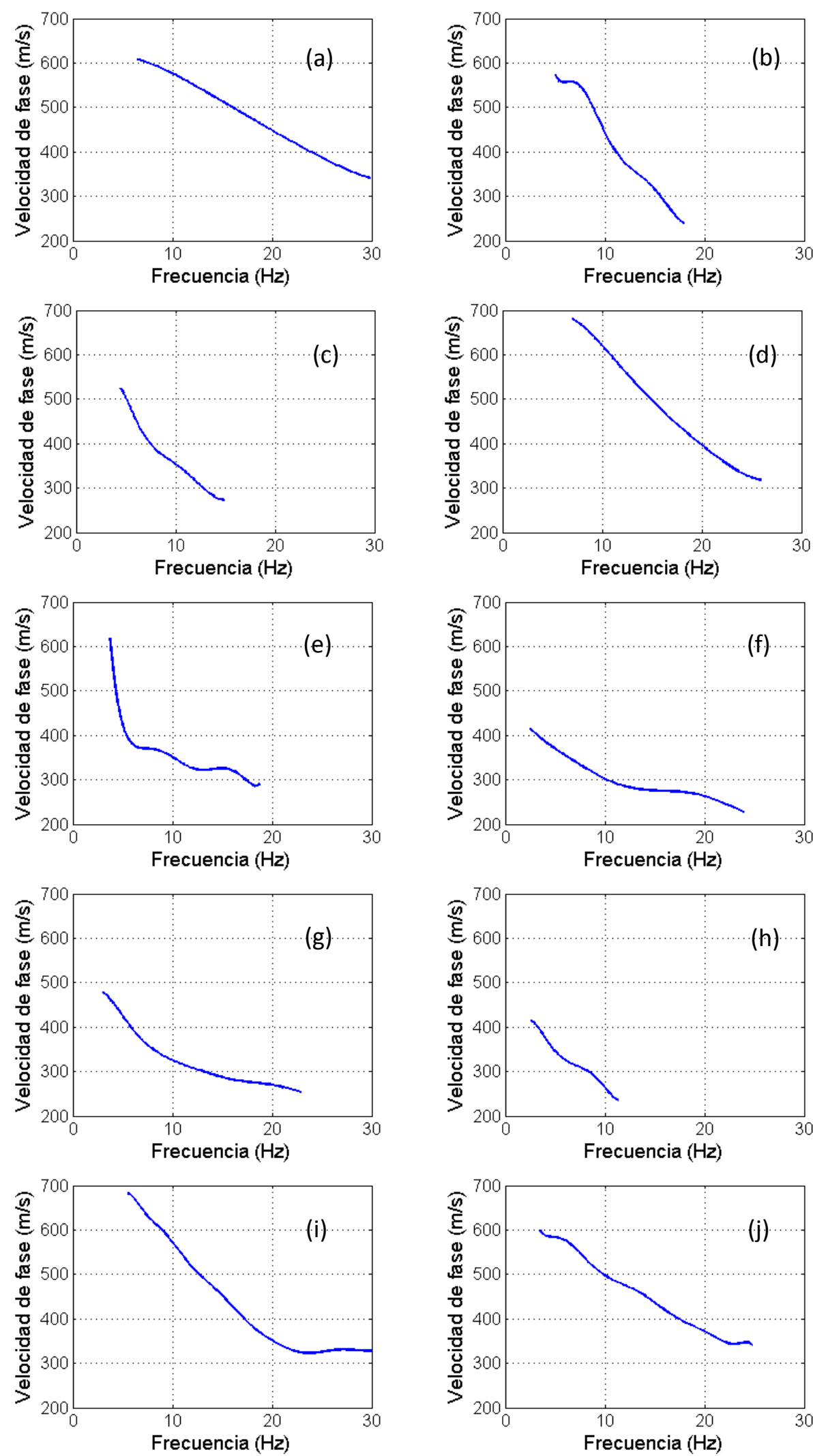

Figura 3.1 Curvas de dispersión obtenidas en Granada a) Aynadamar; b) Zaidín; c) Beiro; d) Bola de Oro; e) Fuentenueva; f) Estadio; g)Chana; h) Náyades; i)Ciencias; j) Granada 74. 


\subsection{Método de la razón espectral HVSR.}

\section{Descripción del método HVSR de microtremor.}

La técnica implica que los microtremores están compuestos principalmente por ondas Rayleigh, producidas por fuentes locales, que se propagan en una capa superficial sobre un semiespacio (Lermo y Chávez-García, 1994). También, considera que el movimiento en la interface entre la capa superficial y el semiespacio no es afectado por el efecto de la fuente, $y$ que el movimiento horizontal y vertical en dicha interface es aproximadamente igual.

La hipótesis de partida de esta técnica (Finn, 1991) es que la energía del microtremor consiste principalmente en ondas Rayleigh y la amplificación de sitio se debe a la presencia de una capa de suelo blando sobre un semiespacio. Tenemos 4 componentes de movimiento, dos en la capa de suelo y dos en el semiespacio. De acuerdo con Nakamura (1989), es posible estimar el efecto de la amplitud de la fuente con la razón:

$$
A_{S}=\frac{V_{S}}{V_{B}}
$$

donde $V_{S}$ es el espectro de amplitud de la componente vertical en la superficie y $V_{B}$ es el espectro de amplitud de la componente vertical en el semiespacio. Después se define una estima del efecto de sitio $S_{E}$ :

$$
S_{E}=\frac{H_{S}}{H_{B}}
$$

donde $H_{S}$ es el espectro de amplitud de la componente horizontal en la superficie y $H_{B}$ es el espectro de amplitud de la componente horizontal en el basamento (la base rígida de la capa de suelo). Para compensar $S_{E}$ por el efecto de la fuente, se calcula una función modificada de efecto de sitio:

$$
S_{M}=\frac{S_{E}}{A_{S}}
$$


Que es equivalente a:

$$
S_{M}=\frac{H_{S} / V_{S}}{H_{B} / V_{B}}
$$

Finalmente se acepta que la relación $H_{B} / V_{B}$ es igual a la unidad (propiedad comprobada por Nakamura en pozos con instrumentación), y la función del efecto de sitio, corregida por el término de la fuente, se puede escribir como:

$$
S_{M}=\frac{H_{S}}{V_{S}}
$$

La suposición de que el radio $H_{B} / V_{B}$ es igual a la unidad fue verificado por Nakamura experimentalmente, usando mediciones de microtremor en lo profundo de un pozo.

\section{Cómputo de la razón espectral HVSR.}

En términos generales podemos decir que el cómputo de la razón espectral H/V sigue los siguientes pasos:

- Registro de las tres componentes de la señal de ruido sísmico ambiental.

- Selección de ventanas estacionarias en el tiempo, pudiéndose usar algún algoritmo de anti-disparo (anti-triggering) con el fin de evitar en el análisis ruido transitorio.

- Cálculo y suavizado de la amplitud del espectro de Fourier para cada ventana.

- Promedio de las dos componentes horizontales

- Cálculo de la razón H/V para cada ventana

- Cálculo del promedio de la razón H/V

Para obtener las curvas se hizo uso del Programa Geopsy y de dos programas generados en Matlab, de los cuales se habla en las siguientes páginas. Los resultados finales que se muestran en la Tabla 3.1 se obtuvieron con el programa Geopsy. La razón de hacer también el estudio con los otros dos programas fue la de corroborar los resultados y poder observar las diferencias en los métodos de cálculo de la razón espectral HVSR. 


\section{Programa HVSR Pwelch.}

El programa aquí llamado HVSR Pwelch (Anexo A) es una serie de instrucciones en Matlab que hacen un proceso similar, aunque más sencillo, al que realiza Geopsy (descrito más adelante). Este programa fue desarrollado por el profesor Gerardo Alguacil (UGR) y modificado por el autor en la parte referente al cálculo del promedio de las componentes horizontales.

El programa hace uso de la función Pwelch de Matlab. Esta función estima la densidad espectral de potencia del vector de entrada usando el método de la estimación espectral de periodograma promedio de Welch. El algoritmo hace una segmentación del vector de entrada en secciones de igual longitud con una superposición del $50 \%$ que puede ser modificada por el usuario. Cada segmento es delimitado por una ventana "Hamming" que es de la misma longitud del segmento original.

Para obtener la razón espectral entre las componentes horizontales y verticales de movimiento, la componente horizontal se calcula mediante la media cuadrática de las dos componentes horizontales, de igual forma que lo hace el programa Geopsy. La diferencia radica en que no es necesario elevar al cuadrado los vectores ya que la función Pwelch da como resultado la potencia espectral.

$$
H / V=\sqrt{\frac{P x x+P y y}{2(P z z)}}
$$

En la Figura 3.2 se muestran dos curvas H/V obtenidas con el programa HVSR Pwelch en dos sitios: Bola de Oro y Calle Aixa la Horra. En ellas se puede apreciar la similitud con las correspondientes a los mismos sitios (Bola de Oro y Calle Aixa la Horra) obtenidas a partir del programa Geopsy y que se muestran en la Figura 3.6 d) y Figura 2.12. Lo anterior es muestra de que el programa Pwelch calcula de forma correcta la razón espectral HVSR, a pesar de la sencillez del método de cálculo utilizado, en comparación con el usado por el programa Geopsy.

\section{Programa HVSR MEM.}

Este programa, al igual que el descrito anteriormente, consiste en una serie de instrucciones en Matlab que fueron desarrolladas por el Profesor Gerardo Alguacil (UGR). En este método se 
hace uso del principio de Máxima Entropía, y comparando con los otros dos métodos no hace uso de una Transformada de Fourier para pasar del domino del tiempo al dominio de la frecuencia.

Una señal aleatoria, como lo es el ruido sísmico ambiental, no tiene estrictamente una transformada de Fourier (Lim, 1988). Su descripción en el dominio de la frecuencia se puede hacer mediante su densidad espectral de potencia, pero esta solo puede estimarse, ya que disponemos solo de una muestra de duración limitada.
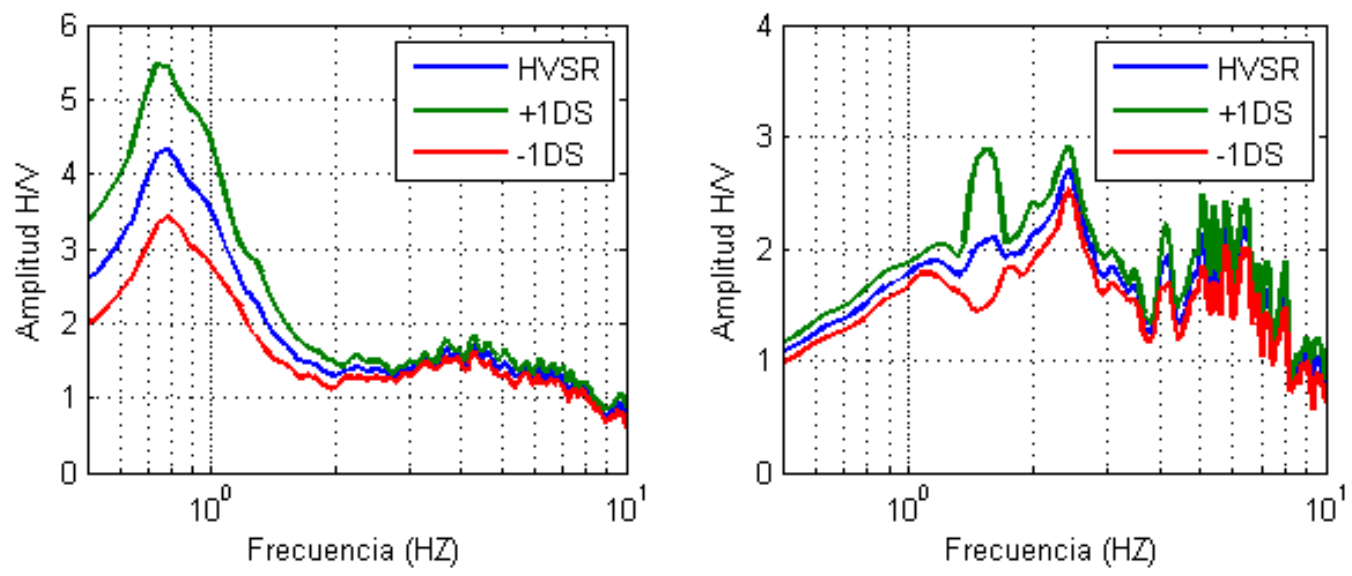

Figura 3.2 Razones espectrales HVSR obtenidas con el programa HVSR Pwelch en Bola de oro (izquierda) y Calle Aixa la Horra (derecha)

El método de la Máxima Entropía (Press et al., 1989) es un método paramétrico de estimación espectral propuesto por Burg. Este algoritmo ha sido empleado para generar espectros con súper-resolución, lo cual indica que exceden la resolución que en teoría tenían con los datos utilizados. El método compensa la resolución limitada de la transformada de Fourier y resulta muy atractivo para aplicaciones donde las frecuencias están muy próximas y se debe identificarlas. Este método extiende la longitud de sus datos suponiendo el cómo actuaría la función de auto-correlación para grandes desviaciones.

Teniendo una serie temporal estacionaria y sus primeros coeficientes de auto-correlación, el objetivo del método de la máxima entropía es el de obtener el espectro de potencia mediante la determinación del proceso más aleatorio, con los mismos coeficientes de auto-correlación de la serie temporal. En términos de teoría de información, esta es la noción de entropía máxima y de ahí el nombre del método. 
Los resultados obtenidos con este programa fueron bastante buenos cuando el pico de la razón espectral se localizaba por encima de $1 \mathrm{~Hz}$. Sin embargo, cuando la máxima amplitud de la curva HVSR adquiría valores por debajo del hercio el programa era incapaz de reproducir de forma adecuada la curva. Como ejemplo de lo anterior se pude observar la Figura 3.3 en donde las gráficas a), b) y c) muestran un claro pico en frecuencias altas, pero la gráfica c) falla a la hora de encontrar el máximo.
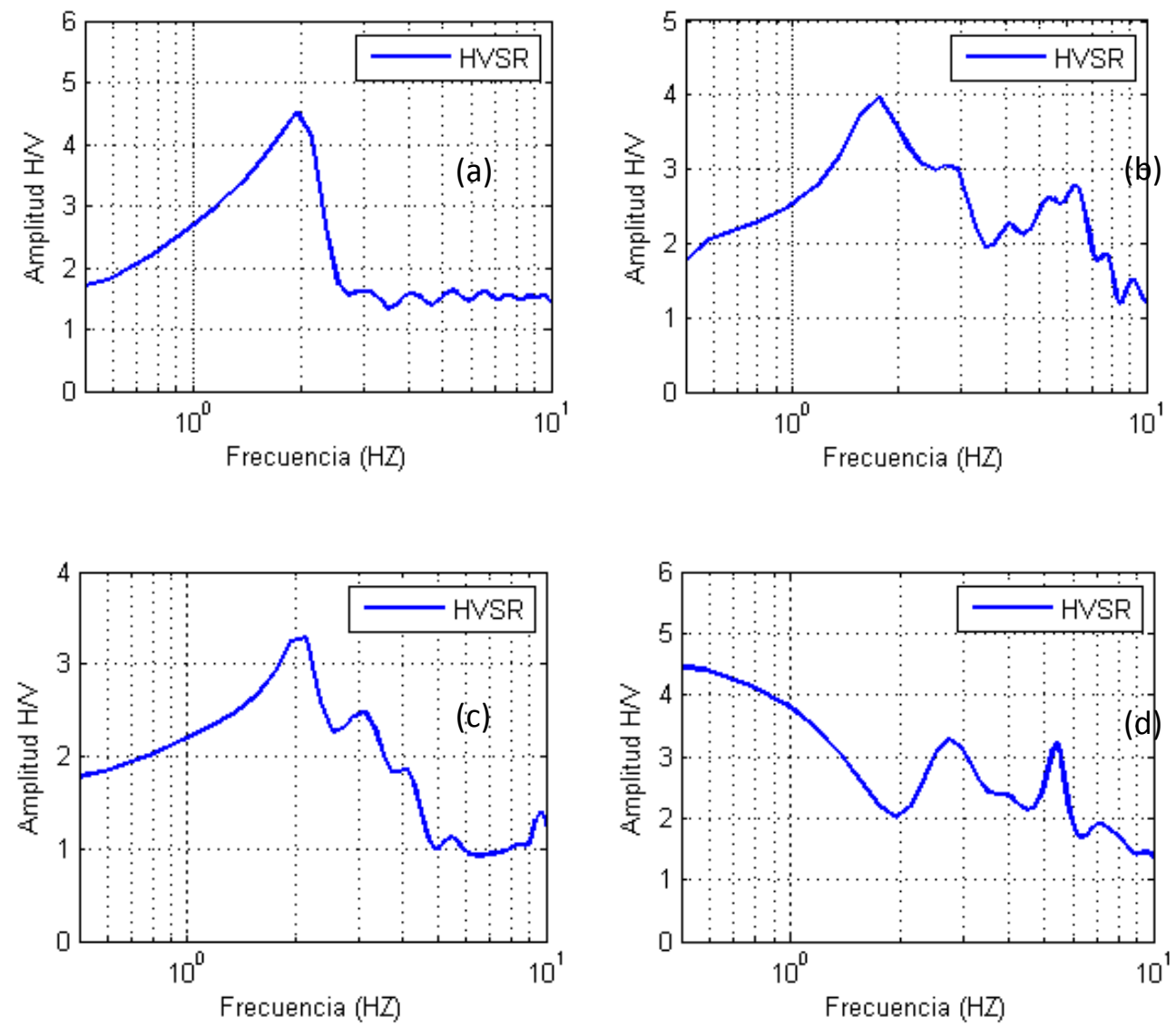

Figura 3.3 Razones espectrales HVSR obtenidas con programa HVSR MEM. a) Fuentenueva b) Calle Aixa la Horra c) Escuela de Caminos d) Campo de los Mártires.

\section{Programa Geopsy.}

Geopsy consta de un conjunto de programas para la investigación y aplicaciones en el área de geofísica. Nació a partir de proyecto europeo SESAME (Site EffectS assessment using AMbient Excitation) y provee, desde 2005 , de herramientas para el procesado de vibraciones ambientales con fines de caracterización de sitios. Dentro de la gama de aplicaciones que contiene Geopsy, fue "geopsyhv" la usada en este punto del trabajo. Dicha aplicación es un 
complemento para cálculo de la razón espectral H/V producto de las contribuciones de J.-L. Chatelian, B. Guillier y P.-Y Bard.

El programa hace posible la elección de una serie de parámetros para el proceso de la señal. El suavizado del espectro de Fourier es encarecidamente recomendado por los que desarrollaron el programa con el objetivo de clarificar el aspecto general de las curvas. El método de suavizado que se ha utilizado en el presente trabajo fue el de Konno y Ohmachi (1998). Este suavizado usa un ancho de banda constante en una escala logarítmica. El suavizado es controlado por una constante que varía entre 0 y 100 . Una constante de valor 0 da un suavizado muy fuerte mientras que una constante 100 hace un suavizado más suave.

Otro parámetro a elegir dentro del proceso de la señal es la función "Taper" (apodización) (Figura 3.4). La función de Taper se usa para minimizar los efectos de borde, debidos a la extracción de una ventana, así como para mejorar sus propiedades en el dominio de la frecuencia. En este trabajo se usó una función Taper del 5\%

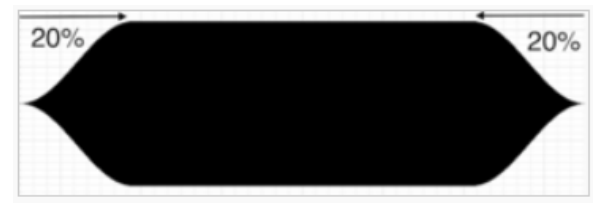

Figura 3.4 Ejemplo de la función Taper al $20 \%$ en el dominio del tiempo.

Dentro de la sección de las componentes horizontales, el programa Geopsy da tres opciones para combinar las componentes Norte-Sur y Este-Oeste de la señal: Media cuadrática, Energía horizontal total y Grados de energía direccional. Para este trabajo fue usada una media cuadrática que utiliza la siguiente fórmula:

$$
H(f)=\sqrt{\frac{N^{2}(f)+E^{2}(f)}{2}}
$$

Se eligieron ventanas con una duración variable de entre 20 y 40 segundos con un solape del 30\% (Figura 3.5). Lo anterior no influye en la máxima amplitud espectral pero si mejora el aspecto general de la curva. Los parámetros usados en el algoritmo de anti-disparo fueron los siguientes: 1 segundo para el promedio a corto plazo o STA ("Short term Averaging"), 30 
segundos para el promedio a largo plazo o LTA ("Long term Averaging"), 0.20 como valor mínimo de la razón STA/LTA y 2.5 como valor máximo de la razón STA/LTA.

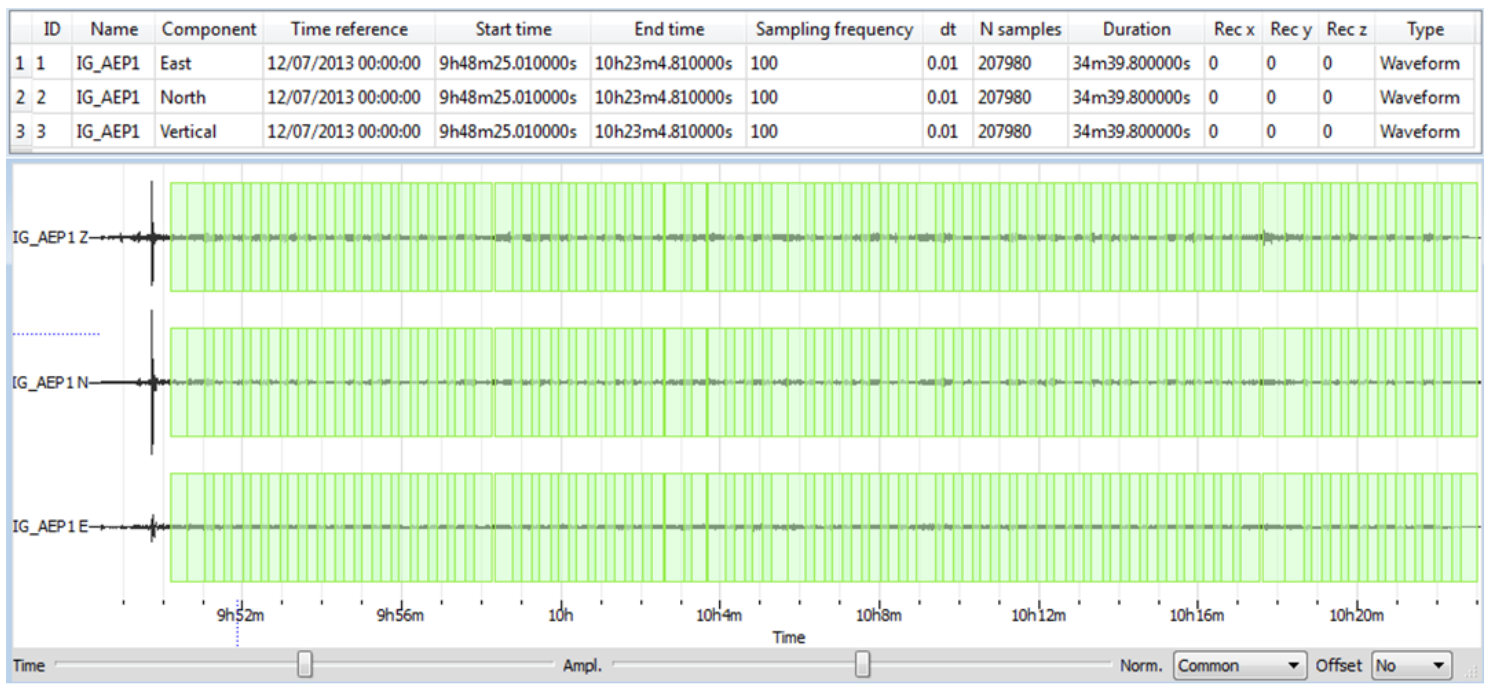

Figura 3.5 Ejemplo de señal en Geopsy con algoritmo de anti-disparo, en verde se muestran las ventanas útiles para el cálculo de la razón espectral HVSR. Sitio Granada 74.

Para determinar si la curva $\mathrm{H} / \mathrm{V}$ es fidedigna se deben cumplir los siguientes puntos:

i. $f_{0}>\frac{10}{I_{W}}$

ii. $\quad n_{c}\left(f_{0}\right)>200$

iii. $\quad \sigma_{A}(f)<2$ para $0.5 f_{0}<f<2 f_{0}$; si $f_{0}>0.5 \mathrm{~Hz}$.

$O$

$$
\sigma_{A}(f)<3 \text { para } 0.5 f_{0}<f<2 f_{0} ; \text { si } f_{0}>0.5 \mathrm{~Hz} .
$$

En donde:

- $\quad I_{W}=$ longitud de la ventana

- $\quad n_{w}=$ número de ventanas seleccionadas para la curva $\mathrm{H} / \mathrm{V}$ promedio

- $n_{c}=I_{W} \times n_{w} \times f_{0}=$ número de ciclos significativos

- $f=$ frecuencia actual

- $f_{0}=$ frecuencia del pico de la razón $\mathrm{H} / \mathrm{V}$

- $A_{H / V}(f)=$ Amplitud de la curva $\mathrm{H} / \mathrm{V}$ en la frecuencia $f$

- $\sigma_{A}(f)=$ desviación estándar de $A_{H / V}(f)$

En la Figura 3.6 se muestran las razones espectrales HVSR obtenidas en una serie de emplazamientos con el programa Geopsy. Estos resultados se comentan más adelante 

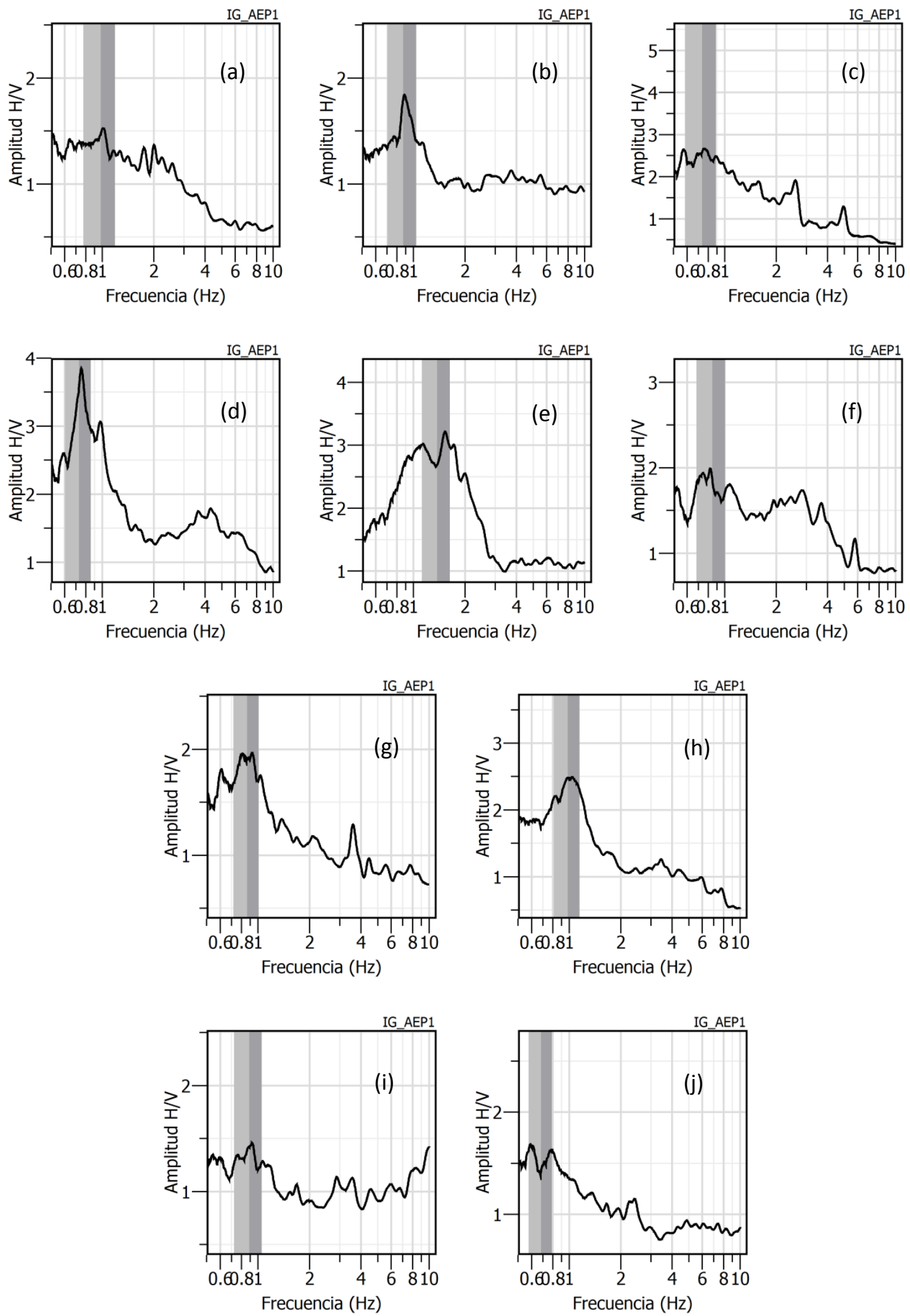

Figura 3.6 Razones espectrales HVSR obtenidas con el programa Geopsy. a) Aynadamar; b) Zaidín; c) Beiro; d) Bola de Oro; e) Fuentenueva; f) Estadio; g) Chana; h) Náyades; i) Ciencias; j) Granada 74. 


\subsection{Análisis y discusión de resultados.}

En la Tabla 3.1 se presentan los resultados del cálculo de la curva HVSR con la técnica de Nakamura. En ella se incluyen los tiempos de medición y las coordenadas de los sitios estudiados. Las frecuencias predominantes en la mayoría de los sitios se aproximan a $1 \mathrm{~Hz}$. Sin embargo, en algunos sitios la amplitud de la razón espectral es cercana a la unidad, por lo que el periodo predominante de los sitios Enfermería, Sanidad y Traductores no es claro. En estos sitios el cálculo fue hecho con muestras de microtremor tomadas con acelerógrafos instalados en los sótanos de los edificios. Como se puede apreciar la respuesta de un acelerógrafo al ruido sísmico ambiental es muy pobre y no entrega buenos resultados con la razón de Nakamura.

Los periodos predominantes de los sitios Calle Aixa la Horra y Campo de los Mártires son de 0.4 segundos para ambos. Este resultado es bastante lógico si se considera que ambos sitios se ubican sobre la Formación Alhambra (Figura 2.7) y por lo tanto el suelo presenta mayor rigidez en comparación con otros puntos de la ciudad. El periodo predomínate del sitio Escuela de caminos es también muy bajo (0.6 s.), sin embargo, aquí el resultado debería tomarse con precaución debido a la cercanía del edificio de la ETSCCP con el punto de muestreo.

Los sitios C. Atlanta, C. Carmen Amaya y C. Náyades se ubican a no más de 200 metros entre sí. La razón de hacer mediciones tan cercanas es la de comprobar que no existe variabilidad de los resultados finales y que son en realidad las características del suelo las que se observan en la curva HVSR. Los periodos predominantes de estos sitios son $1.1 \mathrm{~s} ., 1.2 \mathrm{~s}$ y $1.1 \mathrm{~s}$ para C. Atlanta,

C. Carmen Amaya y C. Náyades respectivamente. Unos resultados tan próximos dan certeza a la medición a pesar de que las amplitudes de las respectivas curvas no son idénticas. Como ya se había discutido, la razón HVSR puede revelar la frecuencia fundamental de un sitio aunque no el nivel de amplificación del movimiento horizontal. Con estos resultados se comprueba que el periodo predominante de la zona de la calle Náyades es de 1.1 segundos aunque su nivel de amplificación es incierto.

En los sitios Bola de Oro, Calle Atlanta, Calle Carmen Amaya, Calle Náyades, Campo del Príncipe, Chana, Estadio de la Juventud, Filosofía, Granada 74, Parque de las ciencias, Beiro y Zaidín los resultados de la frecuencias predominantes de sitio han sido inferiores a $1 \mathrm{~Hz}$. Lo cual indica que estudios previos de microzonificación sísmica como el de Cheddadi (2001) que no hayan contemplado un rango de frecuencias bajo han aportado información incompleta sobre los frecuencias predominantes de la ciudad. 
Tabla 3.1 Resultados del estudio de la razón espectral HVSR en todos los sitios. Incluyendo hora de medición, coordenadas del sitio, Frecuencia dominante, Periodo dominante y Amplitud de la razón HVSR.

\begin{tabular}{|c|c|c|c|c|c|c|}
\hline Sitio & Hora Inicio & Hora Final & $\begin{array}{c}\text { Coordenadas } \\
\text { UTM }\end{array}$ & $\begin{array}{c}\text { Frecuencia } \\
\mathrm{Hz} .\end{array}$ & $\begin{array}{c}\text { Periodo } \\
\text { s. }\end{array}$ & Amplitud \\
\hline Aynadamar & $08 \mathrm{~h} 48 \mathrm{~m} 34 \mathrm{~s}$ & $09 \mathrm{~h} 26 \mathrm{~m} 54 \mathrm{~s}$ & $\begin{array}{l}37.1880^{\circ} \mathrm{N} \\
3.5942^{\circ} \mathrm{W}\end{array}$ & $1.0+/-0.2$ & 1.0 & 1.4 \\
\hline Bola de oro & $06 \mathrm{~h} 55 \mathrm{~m} 22 \mathrm{~s}$ & $07 \mathrm{~h} 27 \mathrm{~m} 53 \mathrm{~s}$ & $\begin{array}{l}37.1644^{\circ} \mathrm{N} \\
3.5849^{\circ} \mathrm{W}\end{array}$ & $0.7+/-0.1$ & 1.4 & 3.5 \\
\hline $\begin{array}{l}\text { C. Aixa la } \\
\text { Horra }\end{array}$ & $01 \mathrm{~h} 00 \mathrm{~m} 00 \mathrm{~s}$ & $02 \mathrm{~h} 00 \mathrm{~m} 00 \mathrm{~s}$ & $\begin{array}{l}37.1609^{\circ} \mathrm{N} \\
3.5789^{\circ} \mathrm{W}\end{array}$ & $2.3+/-0.3$ & 0.4 & 2.6 \\
\hline C. Atlanta & $16 \mathrm{~h} 40 \mathrm{~m} 05 \mathrm{~s}$ & $17 \mathrm{~h} 22 \mathrm{~m} 06 \mathrm{~s}$ & $\begin{array}{l}37.1972^{\circ} \mathrm{N} \\
3.6170^{\circ} \mathrm{W}\end{array}$ & $0.9+/-0.2$ & 1.1 & 2.4 \\
\hline $\begin{array}{c}\text { C. Carmen } \\
\text { Amaya }\end{array}$ & $15 \mathrm{~h} 41 \mathrm{~m} 40 \mathrm{~s}$ & $16 \mathrm{~h} 21 \mathrm{~m} 55 \mathrm{~s}$ & $\begin{array}{l}37.1948^{\circ} \mathrm{N} \\
3.6170^{\circ} \mathrm{W}\end{array}$ & $0.8+/-0.2$ & 1.2 & 4.7 \\
\hline C. Náyades & $04 \mathrm{~h} 30 \mathrm{~m} 00 \mathrm{~s}$ & 06h00m00s & $\begin{array}{l}37.1955^{\circ} \mathrm{N} \\
3.6165^{\circ} \mathrm{W}\end{array}$ & $0.9+/-0.2$ & 1.1 & 2.6 \\
\hline $\begin{array}{l}\text { Campo de } \\
\text { los Mártires }\end{array}$ & $11 \mathrm{~h} 27 \mathrm{~m} 37 \mathrm{~s}$ & $11 \mathrm{~h} 57 \mathrm{~m} 10 \mathrm{~s}$ & $\begin{array}{l}37.1724^{\circ} \mathrm{N} \\
3.5874^{\circ} \mathrm{W}\end{array}$ & $2.6+/-0.4$ & 0.4 & 1.7 \\
\hline $\begin{array}{l}\text { Campo de } \\
\text { Príncipe }\end{array}$ & $07 \mathrm{~h} 30 \mathrm{~m} 01 \mathrm{~s}$ & $08 \mathrm{~h} 10 \mathrm{~m} 44 \mathrm{~s}$ & $\begin{array}{l}37.1729^{\circ} \mathrm{N} \\
3.5921^{\circ} \mathrm{W}\end{array}$ & $0.8+/-0.2$ & 1.2 & 1.5 \\
\hline Chana & $07 \mathrm{~h} 27 \mathrm{~m} 54 \mathrm{~s}$ & $08 \mathrm{~h} 23 \mathrm{~m} 07 \mathrm{~s}$ & $\begin{array}{l}37.1916^{\circ} \mathrm{N} \\
3.6283^{\circ} \mathrm{W}\end{array}$ & $0.9+/-0.1$ & 1.2 & 1.9 \\
\hline Enfermería & $02 \mathrm{~h} 59 \mathrm{~m} 14 \mathrm{~s}$ & $03 \mathrm{~h} 59 \mathrm{~m} 14 \mathrm{~s}$ & $\begin{array}{l}37.1882^{\circ} \mathrm{N} \\
3.6108^{\circ} \mathrm{W}\end{array}$ & $1.5+/-0.3$ & 0.7 & 1.1 \\
\hline $\begin{array}{l}\text { Escuela de } \\
\text { Caminos }\end{array}$ & $15 \mathrm{~h} 23 \mathrm{~m} 18 \mathrm{~s}$ & $16 \mathrm{~h} 00 \mathrm{~m} 47 \mathrm{~s}$ & $\begin{array}{l}37.1812^{\circ} \mathrm{N} \\
3.6079^{\circ} \mathrm{W}\end{array}$ & $2.1+/-0.3$ & 0.5 & 2.3 \\
\hline $\begin{array}{l}\text { Estadio de la } \\
\text { Juventud }\end{array}$ & $16 \mathrm{~h} 39 \mathrm{~m} 10 \mathrm{~s}$ & $17 \mathrm{~h} 15 \mathrm{~m} 37 \mathrm{~s}$ & $\begin{array}{l}37.1855^{\circ} \mathrm{N} \\
3.6194^{\circ} \mathrm{W}\end{array}$ & $0.8+/-0.2$ & 1.2 & 1.8 \\
\hline $\begin{array}{c}\text { Filosofía y } \\
\text { Letras }\end{array}$ & $08 \mathrm{~h} 41 \mathrm{~m} 40 \mathrm{~s}$ & $09 \mathrm{~h} 39 \mathrm{~m} 44 \mathrm{~s}$ & $\begin{array}{l}37.1919^{\circ} \mathrm{N} \\
3.5959^{\circ} \mathrm{W}\end{array}$ & $0.8+/-0.2$ & 1.2 & 1.8 \\
\hline Fuentenueva & $07 \mathrm{~h} 27 \mathrm{~m} 54 \mathrm{~s}$ & $07 \mathrm{~h} 59 \mathrm{~m} 32 \mathrm{~s}$ & $\begin{array}{l}37.1829^{\circ} \mathrm{N} \\
3.6101^{\circ} \mathrm{W}\end{array}$ & $1.4+/-0.2$ & 0.7 & 2.7 \\
\hline Granada 74 & $09 \mathrm{~h} 48 \mathrm{~m} 25 \mathrm{~s}$ & $10 \mathrm{~h} 23 \mathrm{m0} 0 \mathrm{~s}$ & $\begin{array}{l}37.1977^{\circ} \mathrm{N} \\
3.6047^{\circ} \mathrm{W}\end{array}$ & $0.7+/-0.1$ & 1.5 & 1.4 \\
\hline $\begin{array}{l}\text { Parque de } \\
\text { las Ciencias }\end{array}$ & $09 \mathrm{~h} 15 \mathrm{~m} 30 \mathrm{~s}$ & $09 \mathrm{~h} 46 \mathrm{~m} 05 \mathrm{~s}$ & $\begin{array}{l}37.1609^{\circ} \mathrm{N} \\
3.6096^{\circ} \mathrm{W}\end{array}$ & $0.9+/-0.2$ & 1.1 & 1.5 \\
\hline Beiro & $15 \mathrm{~h} 44 \mathrm{~m} 24 \mathrm{~s}$ & $16 \mathrm{~h} 09 \mathrm{~m} 30 \mathrm{~s}$ & $\begin{array}{l}37.1895^{\circ} \mathrm{N} \\
3.6077^{\circ} \mathrm{W}\end{array}$ & $0.7+/-0.2$ & 1.4 & 2.6 \\
\hline Sanidad & $03 \mathrm{~h} 00 \mathrm{~m} 20 \mathrm{~s}$ & $03 \mathrm{~h} 59 \mathrm{~m} 44 \mathrm{~s}$ & $\begin{array}{l}37.1870^{\circ} \mathrm{N} \\
3.6130^{\circ} \mathrm{W}\end{array}$ & $1.5+/-0.3$ & 0.6 & 1.2 \\
\hline Traductores & $02 \mathrm{~h} 59 \mathrm{~m} 14 \mathrm{~s}$ & $03 \mathrm{~h} 59 \mathrm{~m} 14 \mathrm{~s}$ & $\begin{array}{l}37.1753^{\circ} \mathrm{N} \\
3.6028^{\circ} \mathrm{W}\end{array}$ & $4.7+/-0.8$ & 0.2 & 1.1 \\
\hline Zaidín & $10 \mathrm{~h} 17 \mathrm{~m} 31 \mathrm{~s}$ & $10 \mathrm{~h} 49 \mathrm{~m} 43 \mathrm{~s}$ & $\begin{array}{l}37.1564^{\circ} \mathrm{N} \\
3.5954^{\circ} \mathrm{W}\end{array}$ & $0.9+/-0.1$ & 1.1 & 1.8 \\
\hline
\end{tabular}




\section{Inversión de los modelos de velocidad mediante curvas de dispersión y curvas HVSR.}

En esta sección se calculan modelos de suelo de los puntos en donde se cuenta con información SPAC Y HVSR haciendo uso del complemento "dinver" de Geopsy. Se explica el algoritmo de inversión usado por el programa y se presentan y discuten los resultados.

\subsection{Método de inversión.}

El algoritmo de inversión usado por el programa Geopsy fue propuesto por Wathelet (2007). Se trata de un esquema de inversión directa para curvas observadas con el fin de obtener el perfil de velocidades Vs. Se usa para ello el Algoritmo Vecino ("neighborhood algorithm") (Sambridge, 1999) que hace eficiente la búsqueda de todas las soluciones en un espacio multidimensional de parámetros.

El Algoritmo Vecino (Wathelet, 2007) es un método estocástico de búsqueda directa para encontrar modelos de datos aceptables que se ajusten a un espacio multidimensional de parámetros (Sambridge, 1999). El algoritmo se basa en la división del espacio de parámetros en celdas de Voronoi. Las celdas de Voronoi son una descomposición única del espacio en $n$ celdas alrededor de $p_{i}$ puntos. Cada punto dentro de una celda Voronoi representa entonces una muestra, la cual a su vez es un grupo de parámetros que corresponde a un modelo de suelo. La celda alrededor del punto $p_{i}$ es definida por el ensamble de los puntos con mayor cercanía a $p_{i}$ que cualquier otro punto $p_{j}$, en donde $i \neq j$. El objetivo es muestrear todas las regiones del espacio de parámetros en donde se encuentran modelos con error aceptable. La descomposición Voronoi del espacio de parámetros es la base de la función de desajuste del problema inverso, la cual es progresivamente refinada durante el proceso de inversión.

El Algoritmo Vecino necesita cuatro parámetros de afinación: el número de iteraciones por ejecución; el número de modelos escogidos aleatoriamente dentro del espacio de parámetros al inicio de la inversión; el número de modelos a generar en cada iteración; y el número de celdas destacadas en donde se generan los nuevos modelos.

La inversión sigue los siguientes pasos. 1) Los modelos iniciales son generados aleatoriamente con una probabilidad uniforme dentro del espacio de parámetros. 2) La función de desajuste es calculada para los primeros modelos. 3) Las mejores celdas son seleccionadas. 4) Se generan 
los nuevos modelos dentro de las mejores celdas con una probabilidad uniforme en cada celda. 5) Por último, las nuevas muestras regresan al cálculo de la función de desajuste y el proceso se repite el número de iteraciones señalado.

En el caso de que una nueva celda sobresaliente se ubique en los márgenes de la celda del cálculo anterior, la densidad de muestreo no decrecerá sistemáticamente después de la iteración. Esta es una propiedad de la geometría de Voronoi que permite al centro de muestreo saltar de un sitio a otro, mientras que se muestran las regiones más prometedoras simultáneamente.

La inversión conjunta de la curva de dispersión (obtenida con SPAC) y la curva HVSR se hace con el fin de que la información de ambas observaciones sea complementaria. Como hemos visto, la razón HVSR generalmente exhibe un pico que corresponde a la frecuencia fundamental del sitio. Aun y cuando no es bien conocido el contenido del campo de ondas del ruido sísmico ambiental, aquí se hace la suposición de que las ondas superficiales Rayleigh son las predominantes, por lo que su elipticidad dicta las curvas observadas. El espectro H/V contiene información valiosa acerca de la estructura subyacente, especialmente una relación particular entre la velocidad Vs de los sedimentos y su espesor (Scherbaum et al., 2003). En base a esto, la inversión conjunta genera modelos que se adaptan a la curva de dispersión y que tengan la máxima amplitud de la razón $\mathrm{H} / \mathrm{V}$ en la misma frecuencia de la observada.

Para resolver el problema directo, el cálculo de las curvas de dispersión a partir de los modelos generados, se considera únicamente la velocidad de fase de ondas Rayleigh. Esto es debido a que la curva de dispersión observada es obtenida generalmente procesando la componente vertical del ruido sísmico. El cómputo se basa en el problema de autovalores descrito por Thomson (1950) y Haskell (1953).

Una vez calculadas las curvas de dispersión y las H/V teóricas de los modelos generados en la ejecución del algoritmo, se procede a obtener el valor de desajuste (Figura 4.1 y Figura 4.2). El cual viene dado por la siguiente fórmula.

$$
\text { misfit }_{\text {global }}=(1-\alpha)\left(\text { misfit }_{D C}\right)+\alpha\left(\text { misfit }_{H / V}\right)
$$


Y a su vez:

(4.2)

$$
\text { misfit }_{C D}=\sqrt{\sum_{i=0}^{n_{f}} \frac{\left(x_{d i}-x_{c i}\right)^{2}}{x_{d i}^{2} n_{f}}}
$$

$$
\text { misfit }_{H / V}=\frac{\left(f_{0}\right)_{\text {experimental }}\left(f_{0}\right)_{\text {calculada }}}{\left(d f_{0}\right)_{\text {experimental }}}
$$

En donde:

- $\quad x_{d i}$ es la velocidad de la curva de dispersión observada en la frecuencia $f_{i}$

- $\quad x_{c i}$ es la velocidad de la curva de dispersión calculada en la frecuencia $f_{i}$

- $\quad n_{f}$ es el número de muestras de frecuencias consideradas.

- $f_{0}$ es la frecuencia de la máxima amplitud H/V

- $d f_{0}$ es la desviación estándar de la frecuencia de la máxima amplitud $\mathrm{H} / \mathrm{V}$ experimental

- $\quad \alpha$ el peso dado al desajuste de la razón $\mathrm{H} / \mathrm{V}$

La parametrización del modelo debe incluir todos los casos posibles de estructuras 1D que son capaces de igualar la complejidad de la curva observada. La manera aconsejada por el autor del algoritmo es la de empezar con el modelo más sencillo e ir aumentado las capas necesarias cuando los datos no se adecuan suficientemente. También se aconseja fijar el espesor de las capas con una aproximación de prueba y error dando rangos amplios y enfocándose en las zonas en donde la curva da más información. La densidad del material tiene poca influencia en las curvas de dispersión por lo que se sugiere fijarla en un valor constante por capa basándose en información geotécnica. Los valores de velocidad de onda $\mathrm{P}$ y S son relacionados mediante el módulo de Poisson.

Para parámetros pobremente acotados, los resultados pueden diferir cuando se inician dos inversiones separadas. Entonces, la robustez del resultado final es revisada al ejecutar la misma inversión varias veces con diferentes semillas aleatorias (un valor entero que inicializa el generador pseudo-aleatorio). 

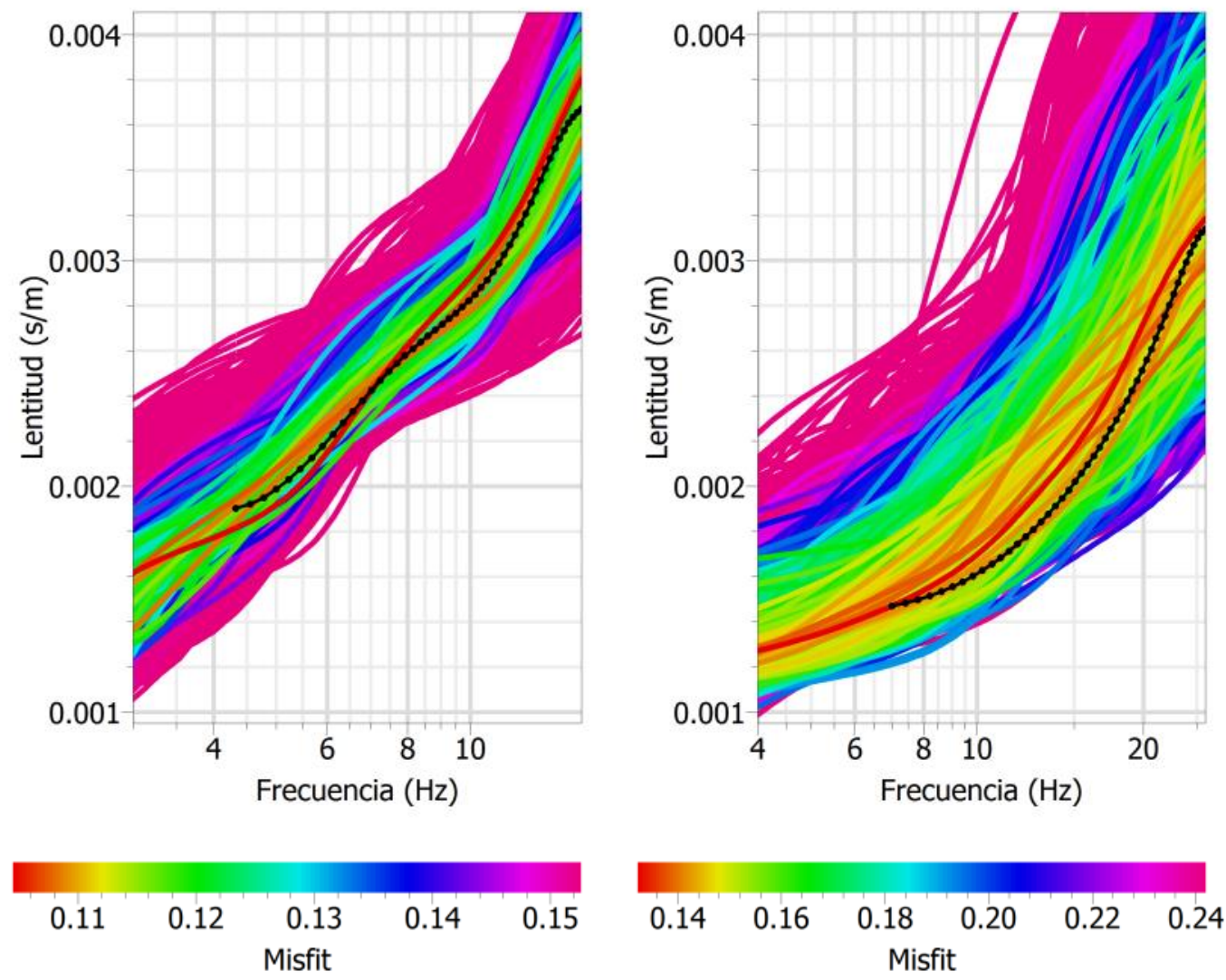

Figura 4.1 Ejemplos de Inversión de la curva de dispersión con valor de desajuste hecha con dinver de Geopsy. Sitio Beiro (izquierda) y Bola de Oro (derecha).
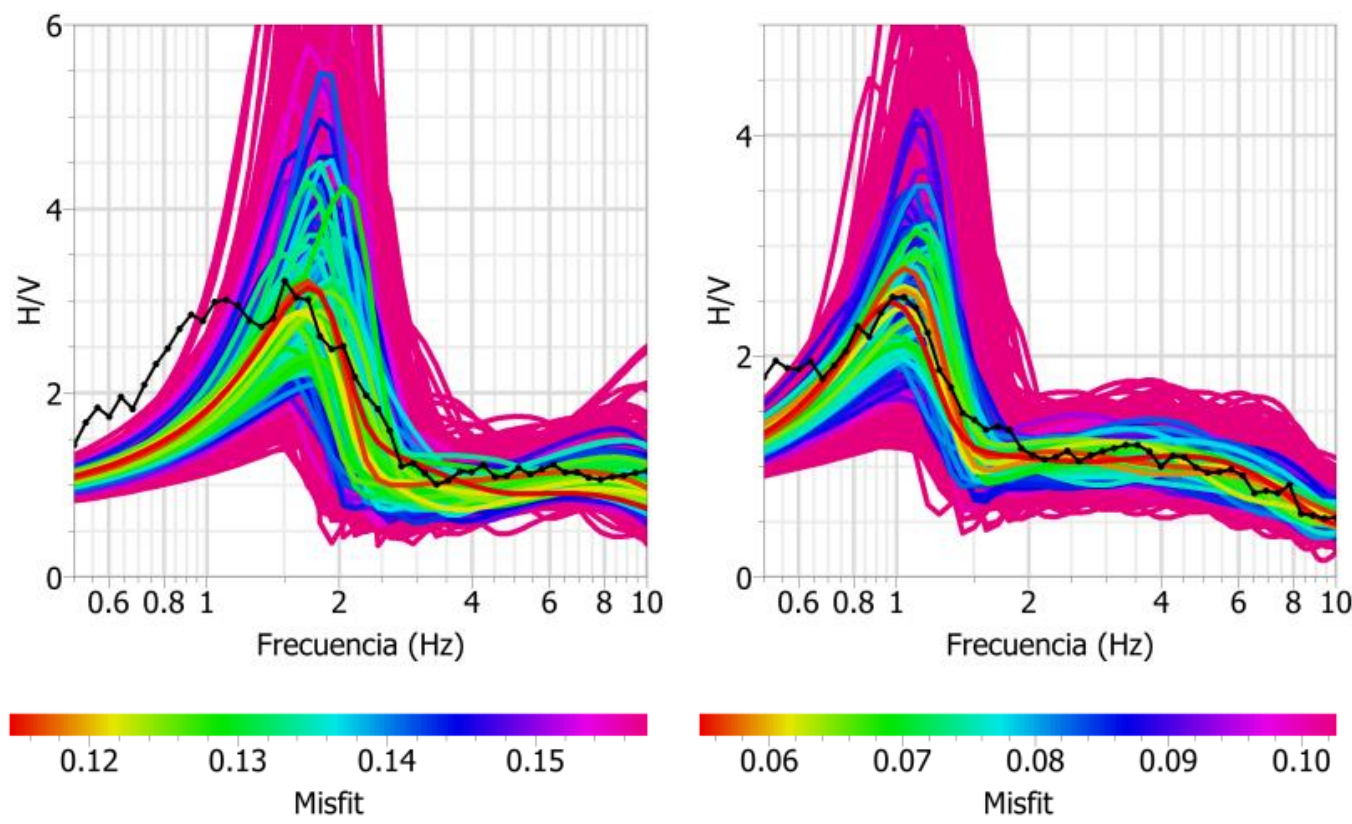

Figura 4.2 Ejemplos de Inversión de la curva $H / V$ con valor de desajuste hecha con dinver de Geopsy. Sitio Fuentenueva (izquierda) y Náyades (derecha). 


\subsection{Análisis y discusión de los resultados de la inversión conjunta.}

En la Tabla 4.1 se presentan los resultados de la inversión conjunta de la curva de dispersión y la curva HVSR con el complemento "dinver" del programa Geopsy. Los parámetros iniciales de los modelos invertidos fueron creados en base a la inversión hecha por Navarro et al. (2012) pero aumentando la profundidad de las capas finales para aprovechar la información de la curva HVSR. La inversión se ejecutó en varias ocasiones con diferentes semillas aleatorias para verificar que los mejores modelos fuesen similares. Haciendo esto se asegura que todo el espacio de parámetros es escaneado en la búsqueda de una solución óptima. También se ajustaron la velocidad de la onda $\mathrm{P}$ y la densidad del material para que variasen conjuntamente con la Vs.

Tabla 4.1 Resultados de la Inversión conjunta hecha con "dinver" de Geopsy. Se presenta el valor de la función de desajuste (Misfit) del mejor modelo por cada sitio.

\begin{tabular}{|c|c|c|c|c|c|c|c|c|c|}
\hline \multicolumn{2}{|c|}{ Aynadamar } & \multicolumn{2}{|c|}{ Zaidín } & \multicolumn{2}{|c|}{ Beiro } & \multicolumn{2}{|c|}{ Bola de Oro } & \multicolumn{2}{|c|}{ Fuentenueva } \\
\hline Misfit & 0.083 & Misfit & 0.080 & Misfit & 0.105 & Misfit & 0.133 & Misfit & 0.115 \\
\hline Prof. & Vs & Prof. & Vs & Prof. & Vs & Prof. & Vs & Prof. & Vs \\
\hline $\mathrm{m}$ & $\mathrm{m} / \mathrm{s}$ & $\mathrm{m}$ & $\mathrm{m} / \mathrm{s}$ & $\mathrm{m}$ & $\mathrm{m} / \mathrm{s}$ & $\mathrm{m}$ & $\mathrm{m} / \mathrm{s}$ & $\mathrm{m}$ & $\mathrm{m} / \mathrm{s}$ \\
\hline 5 & 298 & 6 & 225 & 8 & 234 & 7 & 304 & 6 & 220 \\
\hline 28 & 582 & 19 & 414 & 28 & 410 & 20 & 555 & 30 & 390 \\
\hline 53 & 709 & 69 & 586 & 67 & 673 & 55 & 822 & 71 & 622 \\
\hline 83 & 795 & 94 & 633 & 144 & 706 & 151 & 977 & 140 & 829 \\
\hline \multirow[t]{2}{*}{-} & 1237 & 118 & 713 & - & 1407 & - & 1095 & - & 1489 \\
\hline & & & 986 & & & & & & \\
\hline \multicolumn{2}{|c|}{ Estadio } & \multicolumn{2}{|c|}{ Chana } & \multicolumn{2}{|c|}{ Náyades } & \multicolumn{2}{|c|}{ Ciencias } & \multicolumn{2}{|c|}{ Granada 74} \\
\hline Misfit & 0.077 & Misfit & 0.045 & Misfit & 0.056 & Misfit & 0.114 & Misfit & 0.093 \\
\hline Prof. & Vs & Prof. & Vs & Prof. & Vs & Prof. & Vs & Prof. & Vs \\
\hline $\mathrm{m}$ & $\mathrm{m} / \mathrm{s}$ & $\mathrm{m}$ & $\mathrm{m} / \mathrm{s}$ & $\mathrm{m}$ & $\mathrm{m} / \mathrm{s}$ & m & $\mathrm{m} / \mathrm{s}$ & $\mathrm{m}$ & $\mathrm{m} / \mathrm{s}$ \\
\hline 6 & 236 & 7 & 248 & 10 & 222 & 5 & 248 & 5 & 248 \\
\hline 27 & 349 & 29 & 390 & 34 & 406 & 21 & 495 & 21 & 495 \\
\hline 71 & 475 & 79 & 510 & 69 & 505 & 47 & 706 & 42 & 628 \\
\hline 146 & 639 & 153 & 550 & 117 & 513 & 124 & 831 & 92 & 665 \\
\hline- & 1076 & - & 1212 & - & 1177 & - & 1407 & - & 1131 \\
\hline
\end{tabular}

Estos resultados muestran que la inversión conjunta de la curva de dispersión con la curva H/V aporta información sobre estructuras profundas que el método SPAC con arreglos limitados en la apertura de su radio no proporciona. En una inversión que tan solo contemple las curvas de dispersión aquí estudiadas solo se pueden obtener modelos de hasta 50 metros de 
profundidad (Navarro, et al., 2011), con lo cual sería difícil reproducir las frecuencias predominantes encontradas con el método de Nakamura que se muestran en la Tabla 3.1.

En la Figura 4.3 se muestran esquemáticamente los resultados de la inversión conjunta; en ella se puede apreciar el cambio de Vs con respecto a la profundidad del estrato. En todos los modelos encontrados se llegó hasta la capa con una Vs mayor a $1000 \mathrm{~m} / \mathrm{s}$ aunque la profundidad de esta última capa cambia de sitio en sitio.

Sitios como Aynadamar y Bola de Oro llegan a velocidades altas en poca profundidad, p.e Aynadamar alcanza los $582 \mathrm{~m} / \mathrm{s}$ a 28 metros, mientras Bola de Oro alcanza $555 \mathrm{~m} / \mathrm{s}$ en 20 metros. Aynadamar y Bola de Oro son próximas a la formación Alhambra y eso puede explicar la diferencia con los demás resultados. Estadio, Chana y Náyades son los sitios con Vs más baja en comparación con los demás a profundidades similares. A 69 metros el sitio Náyades tan solo tiene $505 \mathrm{~m} / \mathrm{s}$, mientras que Beiro en 67 ha alcanzado $673 \mathrm{~m} / \mathrm{s}$. La cercanía de los sitios con la Vega de Granada influye en tener estratos menos consolidados que en los sitios próximos a la Formación Alhambra. En una zona intermedia se encuentran Fuentenueva, Beiro, Ciencias y Granada 74 en donde capas con Vs bajas son de menor espesor y se aumenta rápidamente hasta alcanzar velocidades de $800 \mathrm{~m} / \mathrm{s}$ en los últimos estratos.

El sitio de Zaidín está cercano al punto en donde Kagawa et al. (1999) tomaron muestras de microtremor e invirtieron la estructura de suelo con el método SPAC. En la inversión conjunta hecha en el presente trabajo se observa similitud en los resultados para los primeros metros. Aunque el estudio citado alcanzó profundidades de hasta 2 kilómetros por la facilidad de tener arreglos de mucho mayor radio en las afueras de la ciudad. 

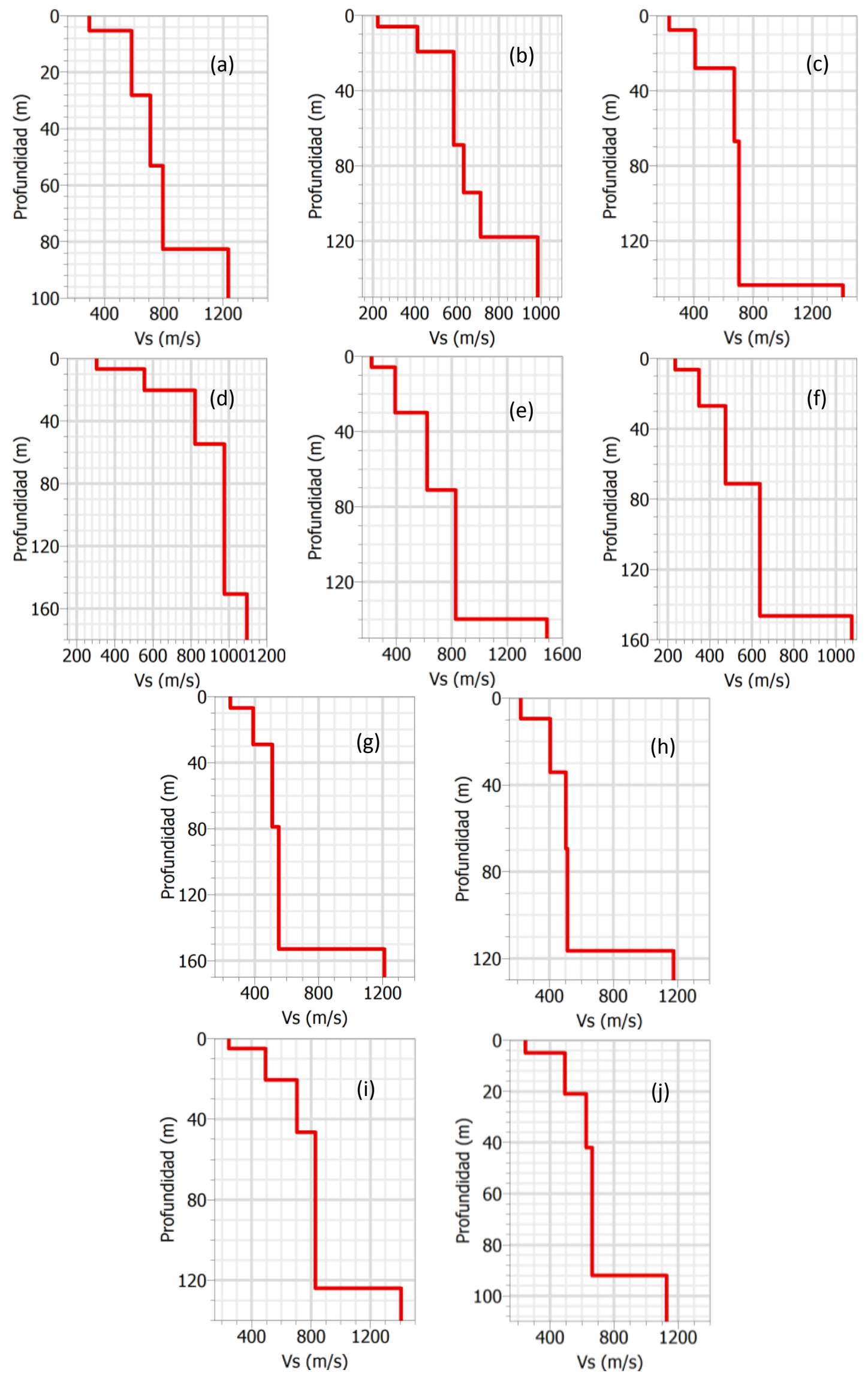

Figura 4.3 Modelos de Velocidad Vs obtenidos de la inversión conjunta de las curvas de dispersión y la razón HVSR a) Aynadamar; b) Zaidín; c) Beiro; d) Bola de Oro; e) Fuentenueva; f) Estadio; g)(hana; h) Náyades; i)Ciencias; j) Granada 74. 


\section{Cálculo de la respuesta dinámica del suelo en la ciudad de Granada.}

En este capítulo se describe la simulación de la respuesta dinámica del suelo realizada para cada uno de los sitios en donde se ha hecho la inversión conjunta del modelo de velocidades Vs. Los terremotos usados como input en la simulación son el de 1980 de Campano-Lucano y el de 2009 de L'Aquila registrados en roca. A partir de los acelerogramas propagados unidimensionalmente a través de la estructura del sitio se calculan parámetros de movimiento sísmico en superficie que caractericen la gravedad de la sacudida sísmica. Dichos parámetros son PGA, PGV, CAV, Espectros de respuesta de aceleración y Espectros de input de energía. La metodología para obtener la respuesta del suelo se detalla a continuación

\subsection{Método para obtener la respuesta del suelo de una estructura del terreno.}

Para obtener la respuesta dinámica del suelo en la ciudad de Granada se realiza una simulación con el programa Degtra (Ordaz y Montoya, 2012) utilizando dos terremotos reales. El primero de ellos corresponde al evento de Campano-Lucano, Italia, del día 23 de Noviembre de 1980 con magnitudes $M_{L}=6.6$ y $M_{S}=6.8$, registrado en la estación RNR en suelo duro y campo libre a una distancia epicentral de 33km (Figura 5.1). El segundo pertenece al del 7 Abril de 2009 de la serie de L'Aquila, Italia, de magnitud $\mathrm{M}_{\mathrm{W}}: 5.3$, registrado a distancia epicentral de $16.7 \mathrm{~km}$ en la estación GSA (Figura 5.2). Se usa el terremoto de L'Aquila para analizar los efectos de aquellos ocurridos en 1431, 1526, 1806, 1911 y 1656 en la proximidad de Granada. Mientras que el terremoto de Campano-Lucano servirá para los de 1884, 1954, 1885 y 1886 en la zona de Alhama (Figura 2.1).

El programa Degtra ha sido desarrollado por M. Ordaz del Instituto de Ingeniería de la Universidad Autónoma de México (UNAM) y C. Montoya Dulché del Centro Nacional de Prevención de Desastres (CENAPRED). Degtra es capaz de visualizar señales y manipularlas usando filtros avanzados para evaluar la coherencia, correlación cruzada, integrar, derivar, calcular espectros de amplitudes de Fourier, entre otras funciones. En el presente trabajo Degtra se utiliza para calcular las funciones de transferencia teóricas (lineales) para los sitios en donde se han calculado los modelos de velocidad Vs. Degtra utiliza el método matricial de propagación de ondas planas en un medio heterogéneo de Thomson Haskell descrito en Aki y Richards (1980). 

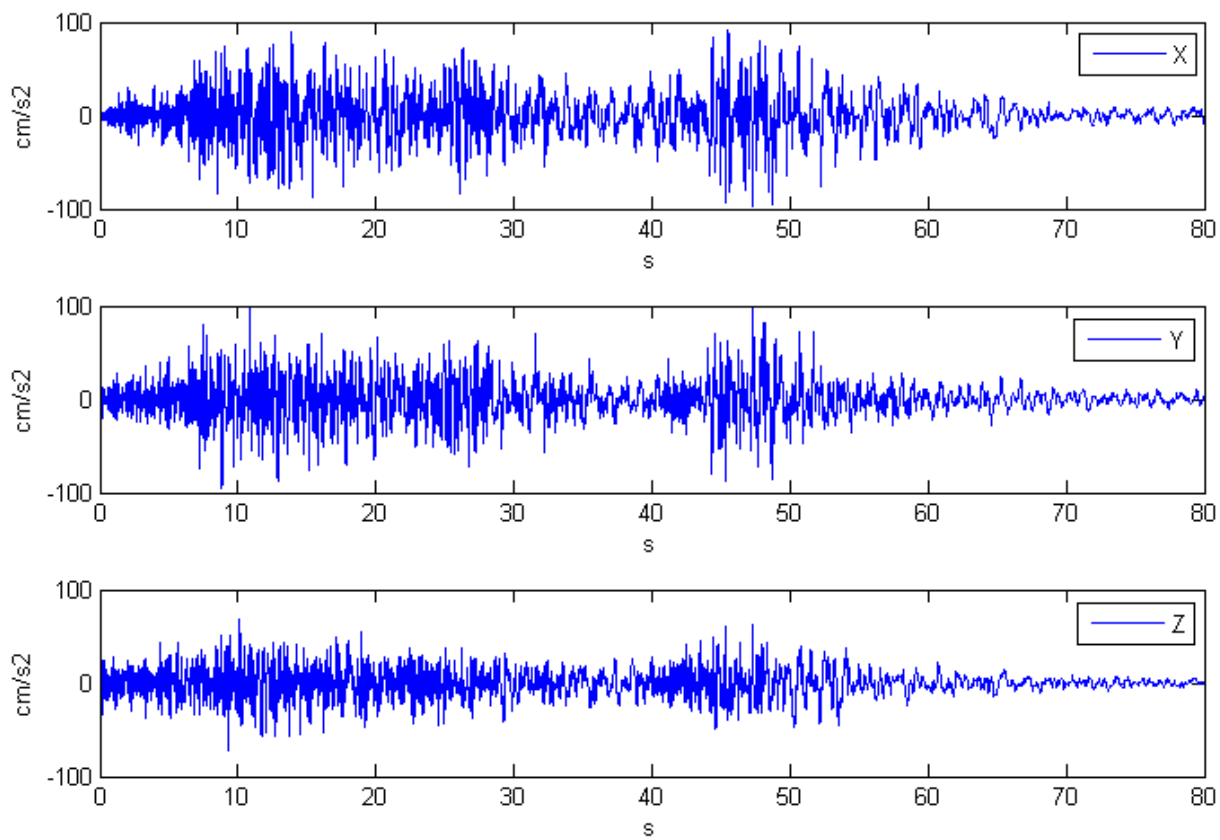

Figura 5.1 Acelerogramas medidos en la estación RNR del terremoto de Campano-Lucano, Italia.
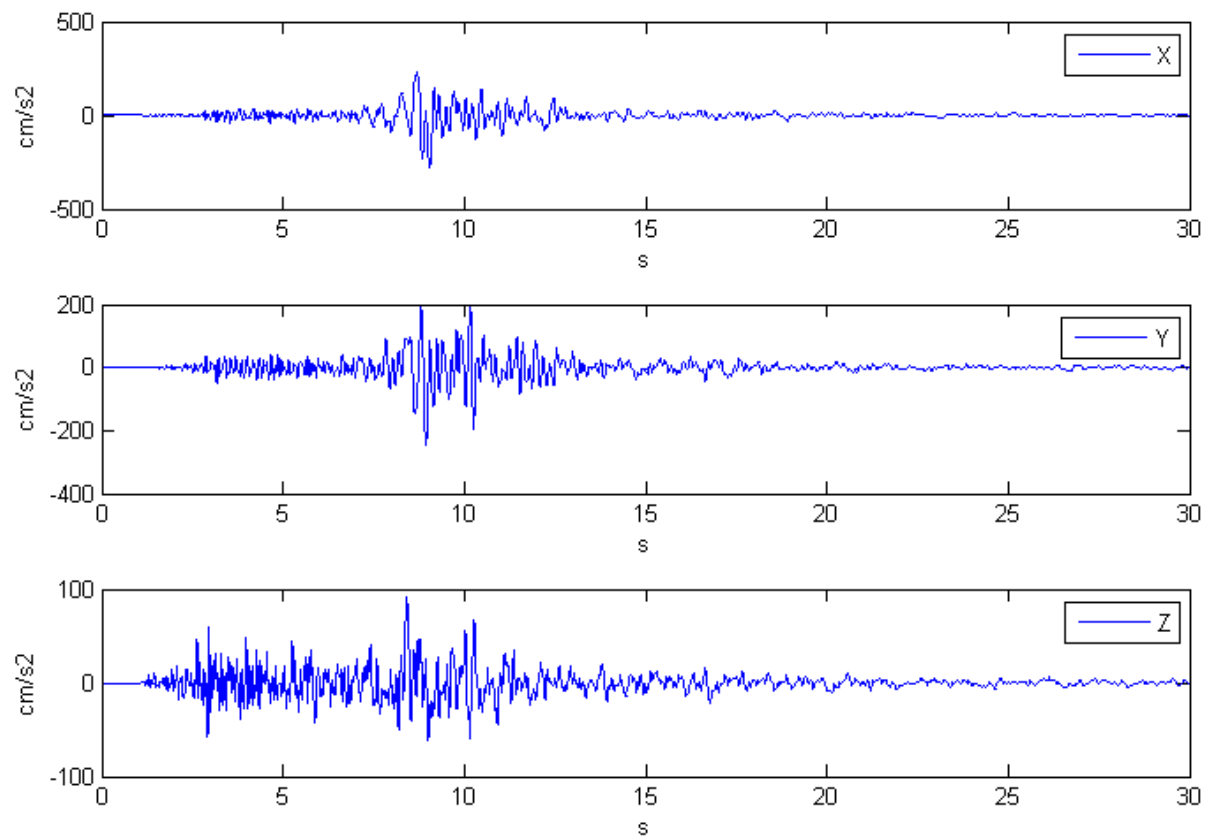

Figura 5.2 Acelerogramas medidos en la estación GSA del terremoto de L'Aquila, Italia.

La función "Propagación unidimensional" de Degtra lleva a cabo una propagación de ondas de corte a través de una estratigrafía con propiedades viscoelásticas. Para hacer el cálculo es necesario dar ciertas propiedades de cada uno de los estratos que componen la columna de suelo. Estas propiedades son la velocidad de onda S, la densidad del material, el porcentaje de amortiguamiento y el espesor del estrato. Se supone que la columna estratigráfica sobreyace a 
un semiespacio elástico, cuyas propiedades deben proporcionarse también. Es necesario además establecer el ángulo de incidencia, ya que es posible realizar el análisis con ondas SH y SV, en éste caso el ángulo de incidencia es nulo. Degtra da la opción de efectuar convolución (el acelerograma de entrada es el campo incidente en la base del depósito de suelo) y deconvolución (el acelerograma de entrada es el campo total en la superficie) en éste caso se ha efectuado la convolución (p.e Figura 5.3 y Figura 5.4).
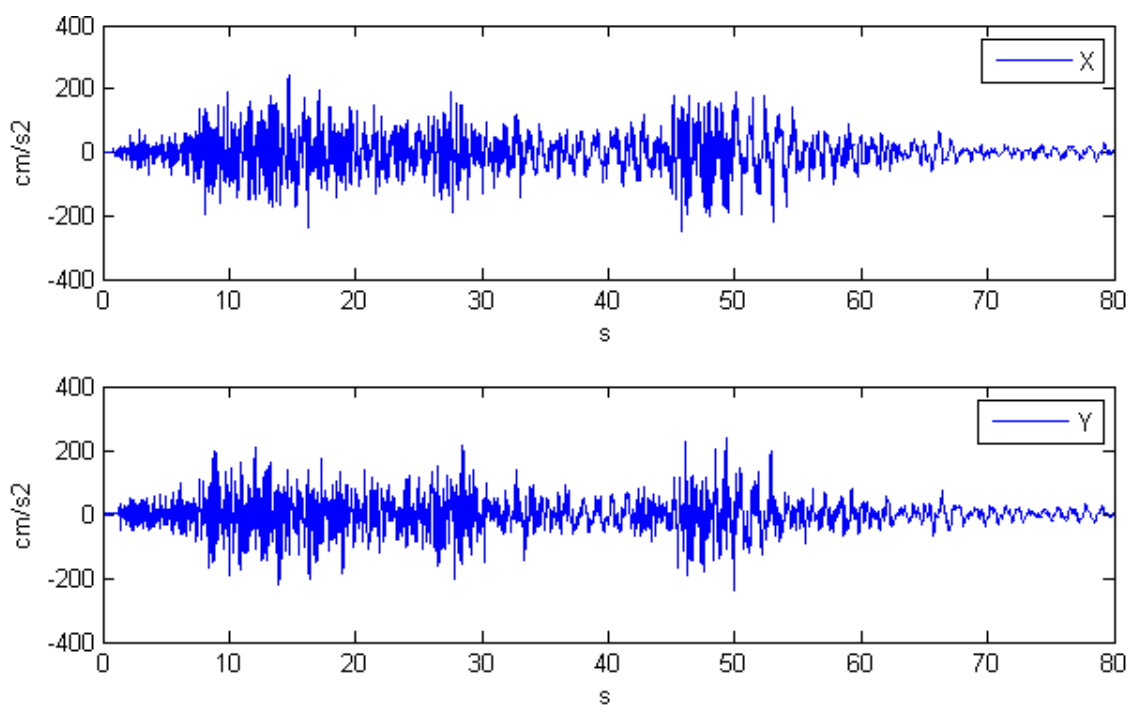

Figura 5.3 Acelerogramas del terremoto de Campano-Lucano propagados unidimensionalmente con el programa Degtra. Sitio Chana.
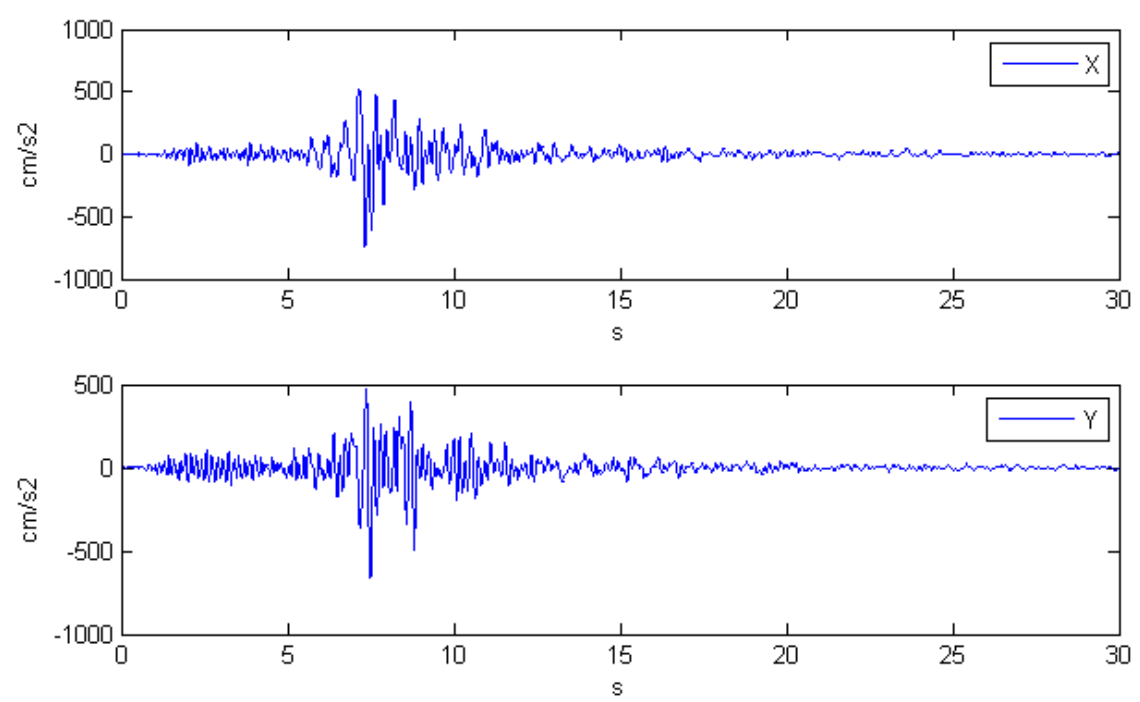

Figura 5.4 Acelerogramas del terremoto de L'Aquila propagados unidimensionalmente con el programa Degtra. Sitio Ciencias. 


\subsection{Parámetros de movimiento sísmico del suelo.}

Los parámetros de movimiento sísmico del terreno que se utilizarán para caracterizar la severidad de la sacudida sísmica en los puntos estudiados son: PGA, PGV, CAV, Intensidad Arias, Espectros de respuesta de aceleración y velocidad, y Espectros de Input de Energía. La importancia de estos parámetros en ingeniería viene de su relación con la acción sísmica sobre las estructuras de edificación (Alguacil y Vidal, 2012).

\section{PGA, PGV, CAV e Intensidad Arias.}

La Aceleración pico del suelo (peak ground acceleration, PGA) y la Velocidad pico del suelo (peak ground velocity, PGV) se usan como indicadores de la intensidad sísmica. EI PGV se obtiene de los acelerogramas integrados respecto al tiempo, ya que la mayoría de sismógrafos se saturan con movimientos intensos del suelo (Alguacil y Vidal, 2012). El valor de PGA y PGV puede referirse al máximo valor del módulo de los vectores de movimiento, al máximo valor en un eje o al máximo valor en el plano horizontal. En este trabajo se considera el máximo valor en el plano horizontal.

Una relación adecuada entre valores de PGA o PGV e Intensidad puede dar paso a la creación de mapas de daño potencial pocos minutos después de ocurrido un terremoto. Los cuales pueden ser usados para respuestas de emergencia, estimaciones de pérdidas y como información para la población afectada. En la actualidad se ha llegado obtener de forma probabilista relaciones reversibles entre Intensidad y los parámetros de movimiento de suelo descritos (Worden, 2012). Estudios recientes favorecen la correlación entre Intensidad y la PGV. Wald et al. (1999) concluyeron que el PGV predice de mejor forma intensidades modificadas de Mercalli superiores a 7, ya que el PGA suele quedar estancado para valores de Intensidad altos.

La velocidad absoluta acumulada (cumulative absolute velocity, CAV) es un parámetro que se propuso originalmente para la definición de una acción sísmica básica operativa en centrales nucleares (EPRI, 1988)

$$
C A V=\int_{t_{0}}^{t_{0}+D}|a(t)| d t
$$


Lo que se interpreta como la sumatoria de las distancias "valle-pico" consecutivas del registro de velocidad, o como el área bajo la aceleración absoluta frente a la duración. En este sentido, tanto la amplitud de la aceleración como la duración contribuyen a este parámetro (Cabañas et al., 2007). En la práctica solo se integran intervalos de $1 \mathrm{~s}$. en los que se supera un determinado umbral, así se eliminan registros de larga duración con aceleraciones bajas. La CAV ha mostrado ser un indicador relevante del potencial de daño y por lo tanto de la intensidad sísmica.

La intensidad de Arias se propuso originalmente para el diseño antisísmico de centrales nucleares como un tensor simétrico.

$$
I_{A i j}=\frac{\pi}{2 g} \int_{t_{0}}^{t_{0}+D} a_{i}(t) a_{j}(t) d t
$$

En la práctica el valor de la Intensidad Arias suele usarse bien en un eje $I_{A i i}$, bien el valor horizontal $I_{A h}=I_{x x}+I_{Y Y}$, o el valor calculado con el módulo de la aceleración al cuadrado.

$$
I_{A}=I_{x x}+I_{y y}+I_{z z}=\frac{\pi}{2 g} \int_{t_{0}}^{t_{0}+D}|a(t)|^{2} d t
$$

El valor en un eje $I_{A i i}$ (Arias, 1970) se obtuvo como la energía por unidad de peso almacenada por una población de osciladores armónicos sin amortiguar en dicho eje de frecuencias propias uniformemente distribuidas entre cero e infinito. La intensidad Arias está asociada con el potencial de licuefacción y de deslizamientos de ladera (Martínez-Rueda y Tsantali, 2008).

\section{Espectros de respuesta de aceleración e input de energía.}

Una forma de caracterizar o definir el efecto de carga de los terremotos sobre las estructuras es mediante espectros elásticos de respuesta. Benavent (2010) define un espectro elástico de respuesta como una representación cartesiana en la que el eje horizontal indica el periodo de vibración (o la frecuencia) de un sistema dinámico de un grado de libertad, y el eje vertical cualquiera de los tres parámetros de respuesta $S_{d}, S_{p v}, \circ S_{p a}$ (desplazamiento espectral relativo, pseudo velocidad espectral relativa y pseudo aceleración espectral relativa respectivamente) 
del sistema frente a un terremoto. Los códigos de construcción antisísmica, como la Norma de construcción sismorresistente española NCSE-02, utilizan espectros promediados, suavizados y normalizados para definir las cargas sísmicas de diseño de los edificios.

Los valores del espectro de respuesta se obtienen de la siguiente manera: Se supone tener un sistema de un grado de libertad fijado al suelo, sometido a una fuerza exterior (equivalente al valor de la masa del sistema por una aceleración externa) con un periodo y un coeficiente de amortiguamiento específico. Mediante la integral de Duhamel se puede resolver la función de desplazamientos de dicho sistema en el dominio del tiempo para varios sistemas con diferentes periodos y así encontrar el máximo desplazamiento espectral relativo. A su vez el $S_{d}$ puede relacionarse por medio de la frecuencia angular con el $S_{p v}$ y $S_{p a}$ siempre y cuando el amortiguamiento sea muy subcrítico (Benavent, 2010). Después de obtener los máximos $S_{p v} 0$ $\mathrm{S}_{\mathrm{pa}}$ de varios sistemas con diferentes periodos de vibración se puede construir el espectro de respuesta para un determinado nivel de amortiguamiento.

En años recientes se ha introducido un nuevo marco para el proyecto sismorresistente de estructuras denominado "Proyecto basado en prestaciones", cuya filosofía es la de preparar la estructura para satisfacer diferentes niveles de comportamiento frente a terremotos de distinta severidad (Benavent, 2010). Dentro de este marco se presenta los métodos energéticos, los cuales son capaces de evaluar cuantitativamente los niveles de daño en las estructuras.

El espectro de input de energía fue introducido por Akiyama (1980), quien demostró que la energía que un terremoto introduce a un sistema de un grado de libertad es un valor muy estable dependiente de la masa y del periodo de vibración. Si se considera el trabajo realizado por una fuerza general externa sobre un sistema de un grado de libertad con una masa $m$, rigidez $k$ y coeficiente de amortiguamiento $c$, se llega a la siguiente ecuación.

$$
\int m \ddot{x} d x+\int c \dot{x} d x+\int f(x) d x=-\int m a(t) d x
$$

En donde se ha sustituido la fuerza elástica por una expresión general $f(x)$ que puede considerar la deformación dúctil. La integral del segundo miembro de la ecuación es el input de energía $E_{l}$ y representa el trabajo realizado por la fuerza de inercia asociado al 
desplazamiento relativo del sistema. El input de energía suele expresarse mediante una pseudo-velocidad equivalente que se define como:

$$
V_{i}=\sqrt{\frac{2 E_{i}}{m}}=\sqrt{2 \int a(t) \dot{x} d t}
$$

Se denomina entonces espectro de input de energía para un terremoto determinado y para un nivel de amortiguamiento dado, a la representación del periodo y la pseudo-velocidad equivalente en unos ejes cartesianos (Benavent, 2010). El cual se puede obtener resolviendo la ecuación de un sistema de un grado de libertad para calcular la velocidad de la masa y así aplicar la ecuación de la pseudo-velocidad equivalente a cada periodo que se desee representar.

\subsection{Características de la respuesta dinámica del suelo.}

\section{Parámetros vectoriales.}

Los valores de PGA y PGV corresponden al máximo valor del módulo de los vectores de movimiento horizontal. La Intensidad Arias se calcula con la ecuación ( 5.3 ), el valor de la aceleración es el módulo de los vectores. De igual forma, la velocidad absoluta acumulada utiliza el módulo de los vectores de aceleración horizontal en la ecuación ( 5.1 ), pero solo son considerados los intervalos de $1 \mathrm{~s}$. en los cuales se supera una aceleración de 0.025 g. Todos los parámetros vectoriales calculados (ver Tabla 5.1) se discuten a continuación.

Los valores de PGA y PGV se comparan en la Figura 5.5. En ella podemos ver una clara diferencia entre los valores alcanzados en los sitios de referencia (medidas en roca) y los sitios de estudio. Los sitios con máximos valores de PGA alcanzados con el acelerograma de Campano-Lucano (C-L) son Náyades y Estadio, que a su vez corresponden a modelos con valores de Vs bajos en capas profundas ( $505 \mathrm{~m} / \mathrm{s}$ a $69 \mathrm{~m}$. y $475 \mathrm{~m} / \mathrm{s}$ a $75 \mathrm{~m}$. respectivamente). Para el mismo terremoto, Aynadamar, cuyos valores Vs son considerablemente más altos que los de Náyades y Estadio, resultó con el menor valor de PGA.

Los valores de PGA para el acelerograma de L'Aquila no presentan grandes diferencias entre los diferentes sitios, salvo Aynadamar en donde el PGA es nuevamente el menor (Figura 5.5). Se puede ver una clara diferencia de PGV entre los 10 sitios con la simulación del terremoto de 
C-L. Estadio, Chana y Náyades son responsables de aumentar en casi el triple el valor del PGV de referencia. Mientras que Aynadamar, Ciencias y Bola de Oro solo aumentan cerca de 7.5 $\mathrm{m} / \mathrm{s}$ con respecto a la referencia. Considerando que Aynadamar y Bola de oro se encuentran relativamente cerca de la Formación Alhambra, valores de PGV tan cercanos al valor del sitio de referencia son coherentes. Los resultados de PGV para el terremoto de L'Aquila (Figura 5.5) guardan una proporción similar entre ellos y rondan el doble del valor medido en el sitio de referencia. Con estos resultados se observa dependencia entre el tipo de suelo y los máximos de velocidad y aceleración alcanzados con la propagación unidimensional del terremoto de Campano-Lucano.

Tabla 5.1 Resultados de PGA, PGV, Al y CAV para los terremotos de Campano-Lucano y L'Aquila en los 10 sitios de estudio y en la referencia.

\begin{tabular}{|ccccc|ccccc|}
\hline \multicolumn{5}{|c|}{ Campano-Lucano } & \multicolumn{5}{c|}{ L'Aquila } \\
\hline Sitio & PGA & PGV & Al & CAV & Sitio & PGA & PGV & Al & CAV \\
\hline cm & $\mathbf{c m} / \mathbf{s}$ & $\mathbf{c m} / \mathbf{s}$ & Sitio & $\mathbf{c m} / \mathbf{s}^{2}$ & $\mathbf{c m} / \mathbf{s}$ & $\mathbf{c m} / \mathbf{s}$ & $\mathbf{c m} / \mathbf{s}$ \\
\hline Referencia & 101 & 11.8 & 112 & 1734 & Referencia & 285 & 15.4 & 48 & 327 \\
Aynadamar & 226 & 18.3 & 461 & 3639 & Aynadamar & 618 & 26.9 & 210 & 742 \\
Zaidín & 275 & 26.2 & 664 & 4448 & Zaidín & 797 & 34.3 & 330 & 890 \\
Beiro & 311 & 23.0 & 753 & 4687 & Beiro & 743 & 33.8 & 310 & 882 \\
Bola de Oro & 250 & 19.3 & 542 & 3911 & Bola de Oro & 771 & 29.9 & 282 & 831 \\
Fuentenueva & 285 & 22.2 & 744 & 4668 & Fuentenueva & 766 & 35.6 & 330 & 912 \\
Estadio & 322 & 31.3 & 743 & 4823 & Estadio & 661 & 34.3 & 277 & 836 \\
Chana & 312 & 33.0 & 656 & 4455 & Chana & 660 & 31.1 & 255 & 788 \\
Náyades & 324 & 29.3 & 807 & 5002 & Náyades & 691 & 34.2 & 251 & 813 \\
Ciencias & 258 & 18.8 & 595 & 4097 & Ciencias & 817 & 31.9 & 283 & 855 \\
Granada 74 & 252 & 19.6 & 582 & 4114 & Granada 74 & 678 & 28.7 & 288 & 863 \\
\hline
\end{tabular}

La velocidad absoluta acumulada de los registros propagados en los 10 sitios de estudio se compara en la Figura 5.6. En el caso del terremoto de L'Aquila los valores de CAV no difieren entre los sitios de estudio, pero muestran una diferencia de hasta 2.8 veces el valor en el sitio de referencia. En este cálculo se integran únicamente los intervalos en los que la aceleración supera un umbral de $0.025 \mathrm{~g}$. El hecho de ser una sumatoria del área bajo la aceleración hace que los valores de CAV sean mayores con la convolución del terremoto de Campano-Lucano que con el terremoto de L’Aquila cuya duración es significativamente menor (Figura 5.1 y Figura 5.2). 


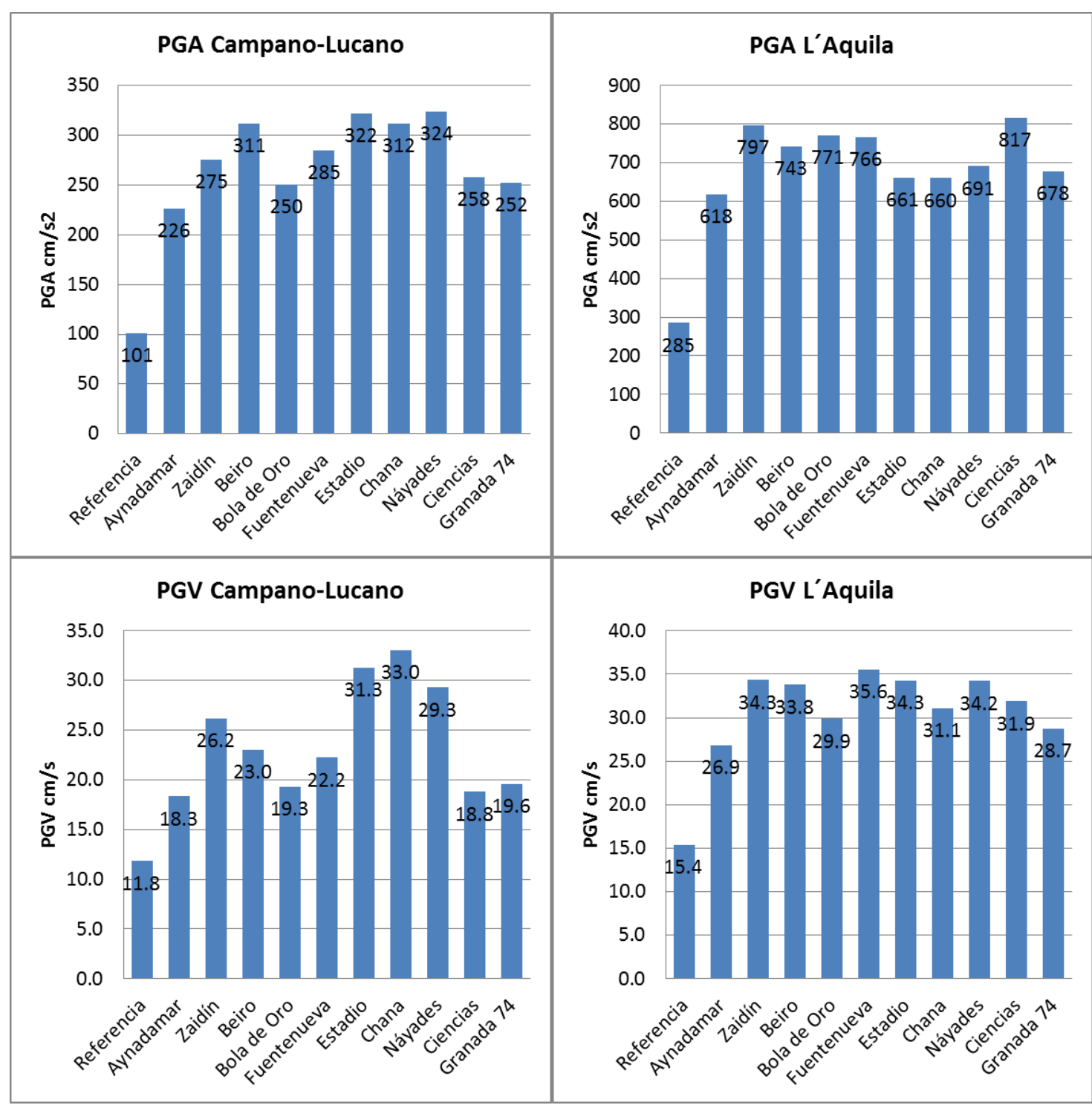

Figura 5.5 Comparación de los valores de PGA y PGV para los terremotos de Campano-Luca y L'Aquila en los 10 sitios de estudio y en el de referencia.

Los resultados de Intensidad Arias se comparan en la Figura 5.7. Al igual que el CAV, la Intensidad Arias es una sumatoria de valores de aceleración, aunque con varias diferencias como el intervalo de integración. Nuevamente los valores de IA para el terremoto de C-L son mayores que los valores para L'Aquila. Cabe mencionar que Náyades es el sitio con los mayores valores de PGA, CAV y AI para la propagación de C-L, sin embargo en la propagación de L’Aquila no existe tal situación. La falta de relación entre los resultados de la propagación de L'Aquila y los modelos de suelo se puede deber a la cercanía de la fuente, el contenido frecuencial y la corta duración del terremoto. 


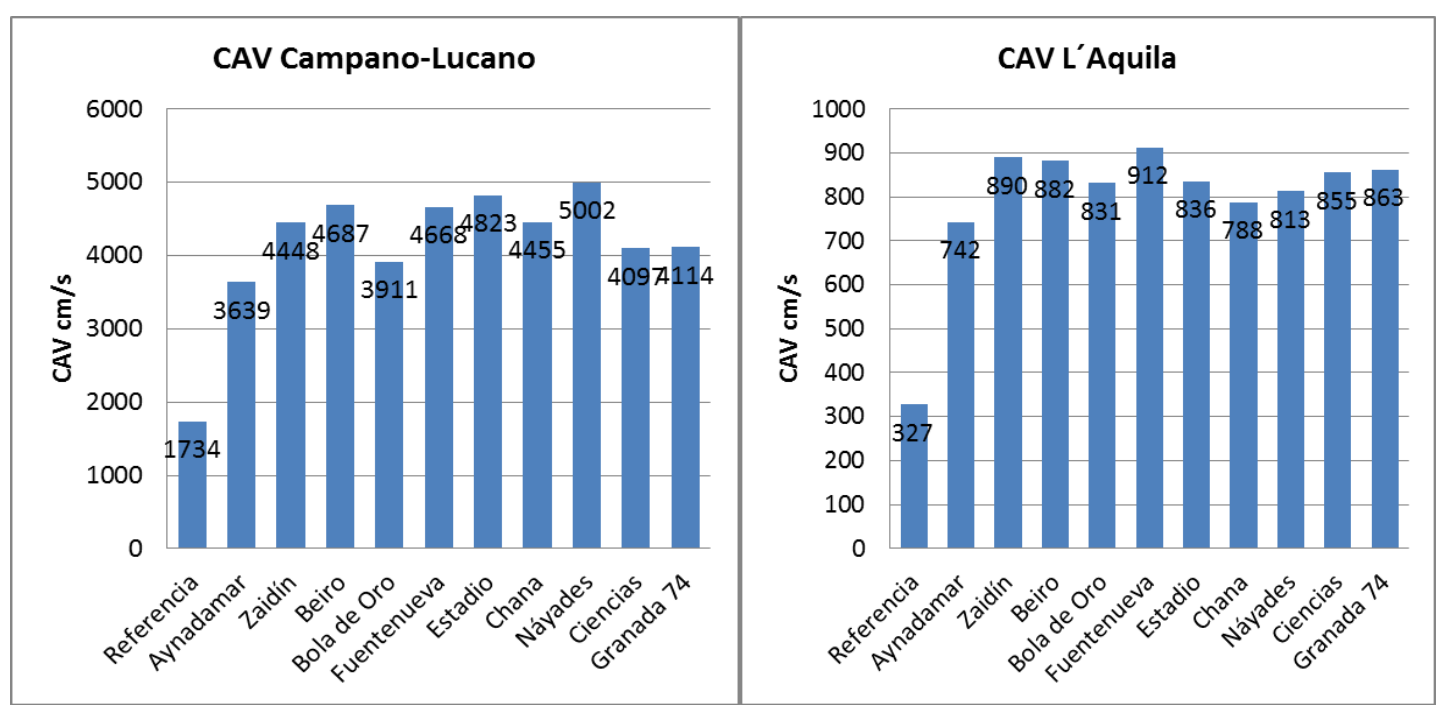

Figura 5.6 Comparación de los valores de CAV para los terremotos de Campano-Luca y L'Aquila en los 10 sitios de estudio y en el de referencia.

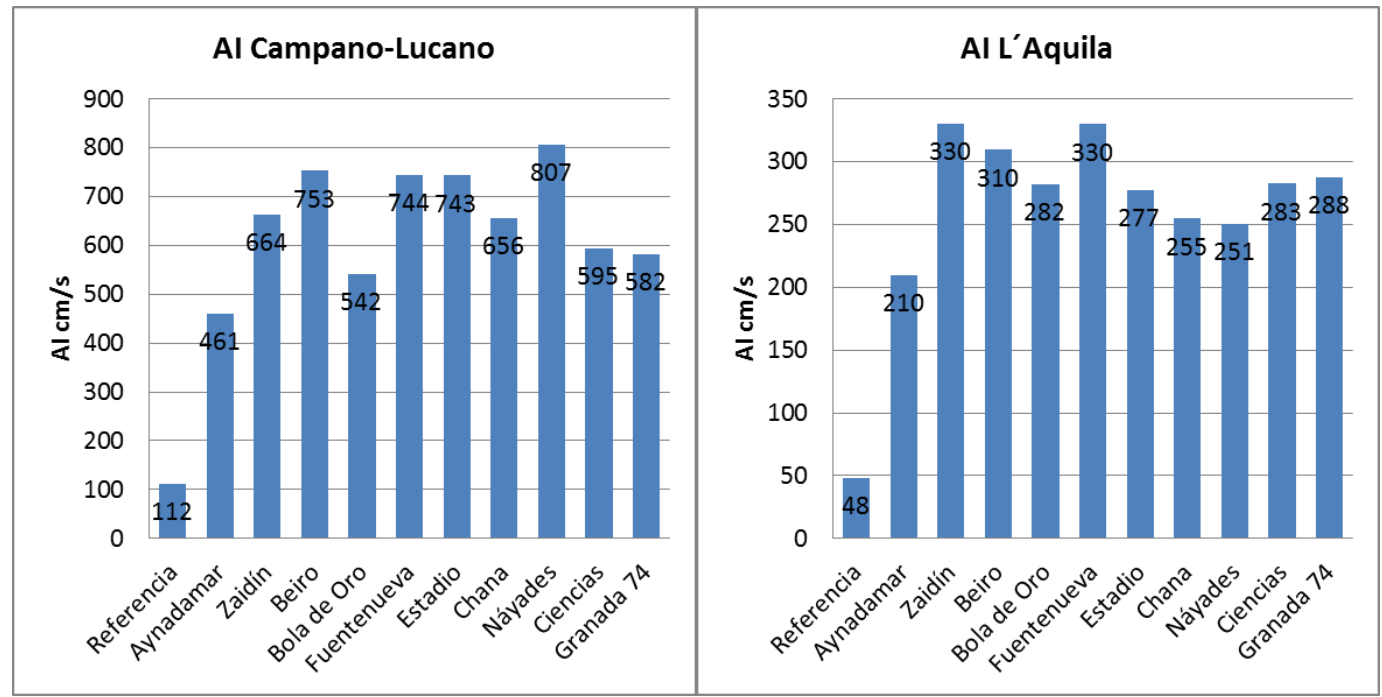

Figura 5.7 Comparación de los valores de Al para los terremotos de Campano-Luca y L'Aquila en los 10 sitios de estudio y en el de referencia.

Alguacil y Vidal (2012) analizaron un conjunto de acelerogramas de la base de datos Internet Site for Strong-Motion Data correspondientes a terremotos del área euro-mediterránea. Analizaron un total de 55 registros con intensidades que varían de VI a VIII en la Escala Macrosísmica Europea (EMS). La media logarítmica de los parámetros vectoriales PGA, PGV, AI y CAV de ese conjunto de terremotos se muestra en la Tabla 5.2. Se observa que un incremento de $P G A$ de $153 \mathrm{~cm} / \mathrm{s}^{2}$ a $262 \mathrm{~cm} / \mathrm{s}^{2}$ puede aumentar en un punto la intensidad registrada. Un cambio similar ocurre con los valores de PGA entre el sitio de referencia y los sitios de estudio para el terremoto de C-L. Similar analogía existe ente el cambio de CAV para las intensidades VII y VIII y la diferencia entre los puntos de estudio y la referencia de la 
propagación del terremoto de L'Aquila. Los valores de IA calculados en este trabajo exceden en su mayoría a los de la Tabla 5.2, al igual que los valores de PGV. Lo anterior no quiere decir que los valores se corresponden con Intensidad IX o X ya que la dispersión de los valores estudiados por Alguacil y Vidal (2012) es muy grande. De cualquier forma se aprecia que el cambio en valor de la media logarítmica de los parámetros vectoriales para hacer que cambie la intensidad observada puede ser producido por el tipo de suelo del sitio.

Tabla 5.2 Media logarítmica de los parámetros PGA, PGV, AI y CAV del conjunto de acelerogramas de terremotos euro-mediterráneos analizados por Aguacil y Vidal (2012).

\begin{tabular}{|ccccc|}
\hline EMS & PGA & PGV & Al & CAV \\
& $\mathbf{c m} / \mathbf{s}^{2}$ & $\mathbf{c m} / \mathbf{s}$ & $\mathbf{c m} / \mathbf{s}$ & $\mathbf{c m} / \mathbf{s}$ \\
\hline VI & 62.0 & 3.6 & 4.76 & 76.6 \\
VII & 153 & 8.3 & 23.2 & 267 \\
VIII & 262 & 21 & 102 & 705 \\
\hline
\end{tabular}

\section{Parámetros espectrales.}

Los espectros de respuesta de aceleración se han calculado aplicando un filtro numérico recursivo a los acelerogramas para obtener las respuestas correspondientes. Lo anterior permite evitar el cálculo de la integral de Duhamel haciendo uso del hecho de que la función de transferencia entre la excitación y la respuesta del sistema es idéntica a la de un filtro de paso-baja de segundo orden (Alguacil y Vidal, 2012). Los espectros de respuesta de aceleración calculados para los dos terremotos se muestran en la Figura 5.8 y la Figura 5.9, que corresponden a sistemas con porcentaje de amortiguamiento con respecto al crítico del $5 \%$. El rango de periodos va de $0.1 \mathrm{~s}$. a $3 \mathrm{~s}$. en intervalos de $0.01 \mathrm{~s}$.

Los espectros de respuesta de aceleración de ambas simulaciones presentan un aumento significativo de aceleración en todos los sitios de estudio. Los espectros de la primera simulación tienen máximos en un rango de periodos de 0.2 a 0.4 segundos con valores que alcanzan los $1600 \mathrm{~cm} / \mathrm{s}^{2}$ en el caso de Náyades y Beiro. De igual forma que con los parámetros vectoriales, el sitio Aynadamar es el que aumenta en menor medida los valores espectrales de la referencia. El terremoto de L'Aquila y las propagaciones de los sitios de estudio tienen espectros de aceleración con energía concentrada en periodos cortos. También es de notar que la forma de los espectros de aceleración para L'Aquila es idéntica tanto en la referencia como en los puntos de estudio. Fuente nueva, Zaidín y Bola de Oro son los tres sitios que más han amplificado la aceleración espectral para este terremoto, mientras que Náyades, Aynadamar y Ciencias aumentan en menor proporción. 


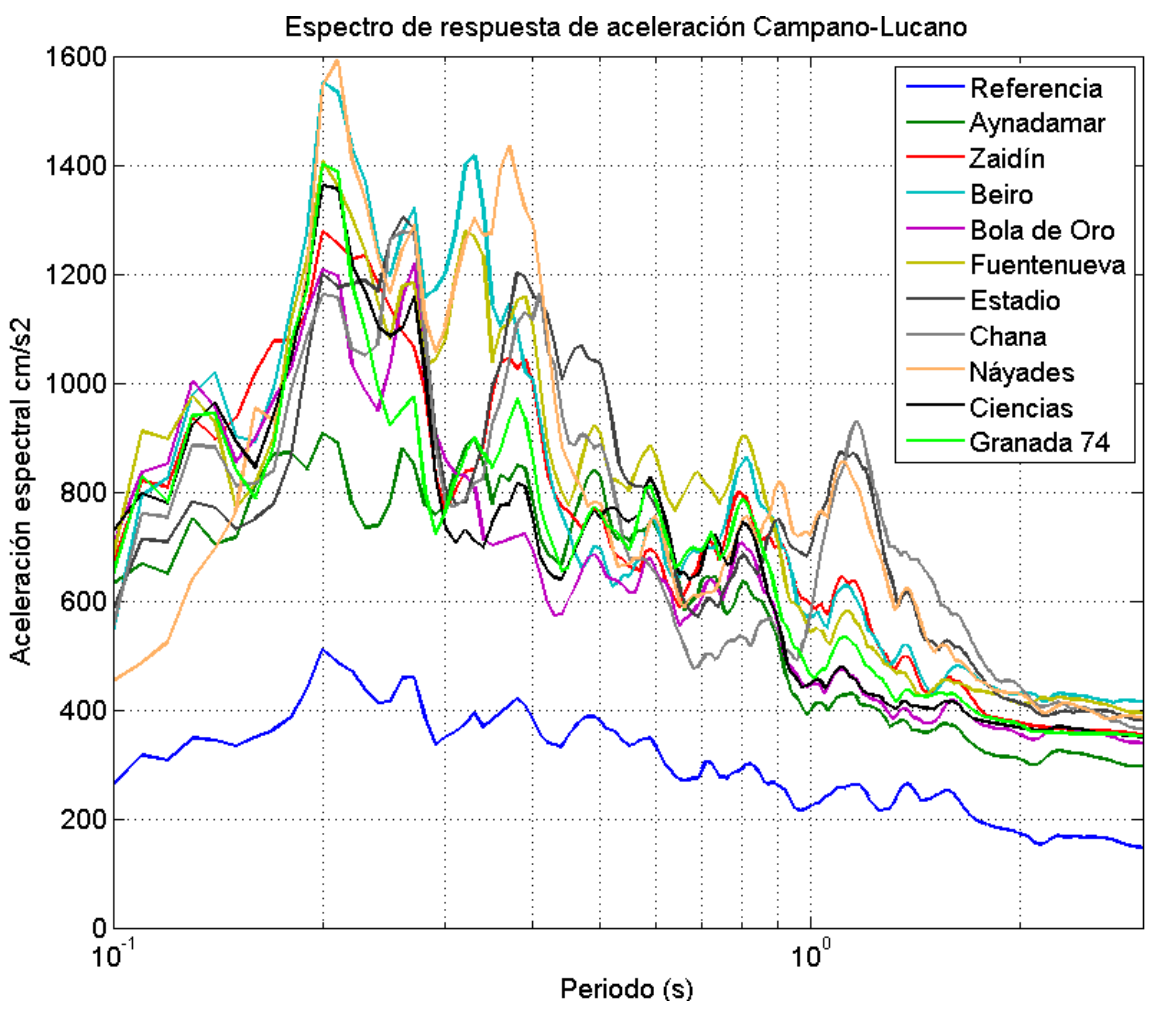

Figura 5.8 Espectros de respuesta de aceleración del terremoto de Campano-Lucano en los 10 sitios de estudio y el de referencia. Puede apreciarse gran diferencia entre los valores en suelo (10 sitios ) y en roca (el de referencia).

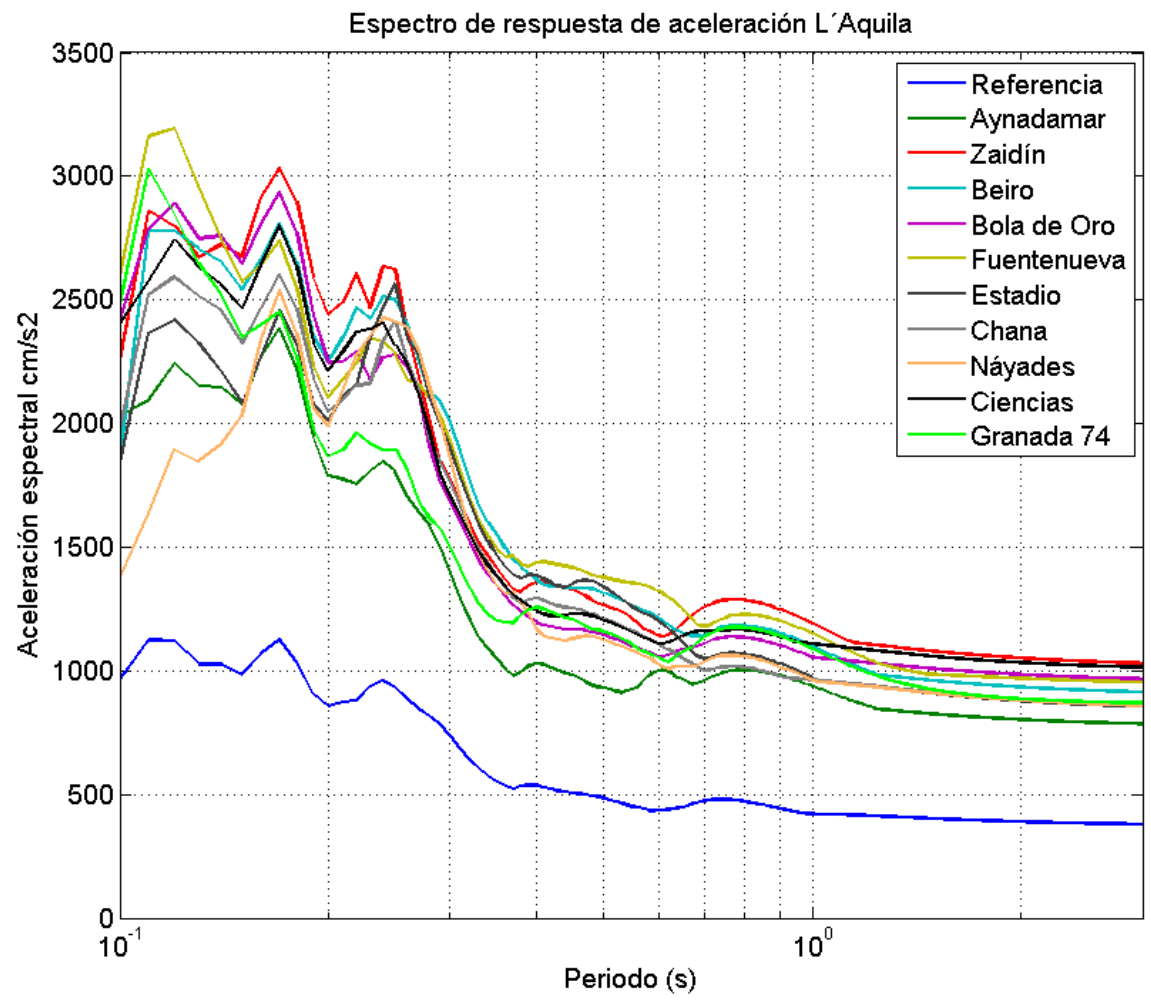

Figura 5.9 Espectros de respuesta de aceleración del terremoto de L'Aquila en los 10 sitios de estudio y el de referencia. Puede apreciarse la gran diferencia entre los valores en suelo (10 sitios) y en roca (el de referencia). 
Los espectros de input de energía se calculan considerando la fuerza recuperadora del sistema en el límite elástico para resolver la ecuación de sistema de un grado de libertad. Se resuelve el sistema aplicando un filtro numérico recursivo y se transforma la respuesta en pseudovelocidad equivalente. El rango de periodos va de $0.1 \mathrm{~s}$. a $3.5 \mathrm{~s}$. en intervalos de $0.01 \mathrm{~s}$

Los espectros de Input de energía calculados para los dos terremotos (Figura 5.10 y la Figura 5.10) muestran nuevamente aumento en la amplitud de la velocidad equivalente para todos los sitios de estudio. En el caso del terremoto de C-L se puede ver que el sitio influye en los rangos en donde aumenta el input de energía con respecto a la referencia. Náyades, Ciencias y Chana presentan un pico en un periodo cercano a 1segundo que es superior a los demás sitios. En el caso del terremoto de L'Aquila los espectros de Input de energía de los 10 sitios reproducen la forma del espectro de la referencia aunque la amplitud aumenta para todos.

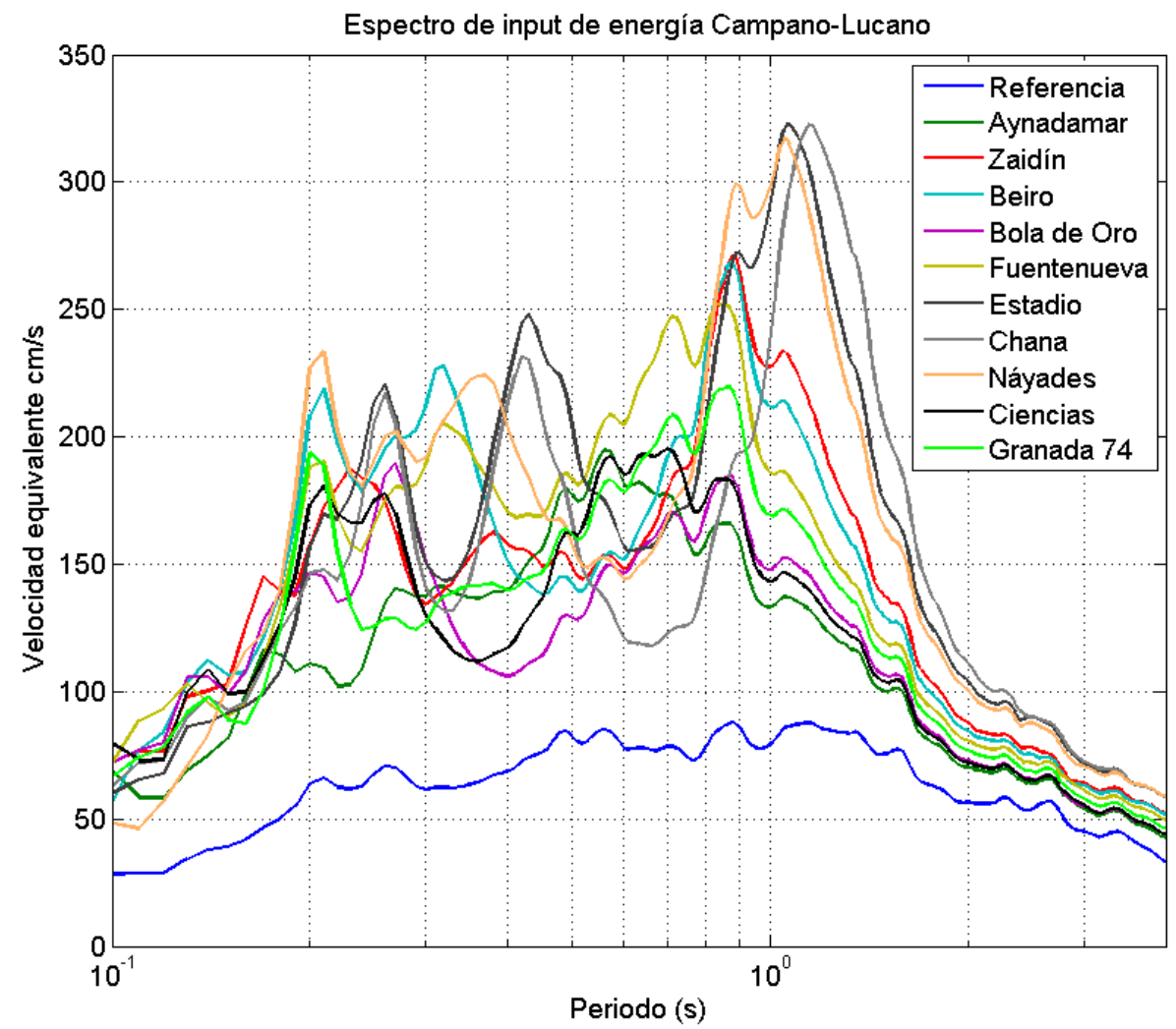

Figura 5.10 Espectros de input de energía del terremoto de Campano-Lucano y el de referencia. Obsérvese la gran diferencia entre los valores en suelo (10 sitios ) y en roca (el de referencia). 


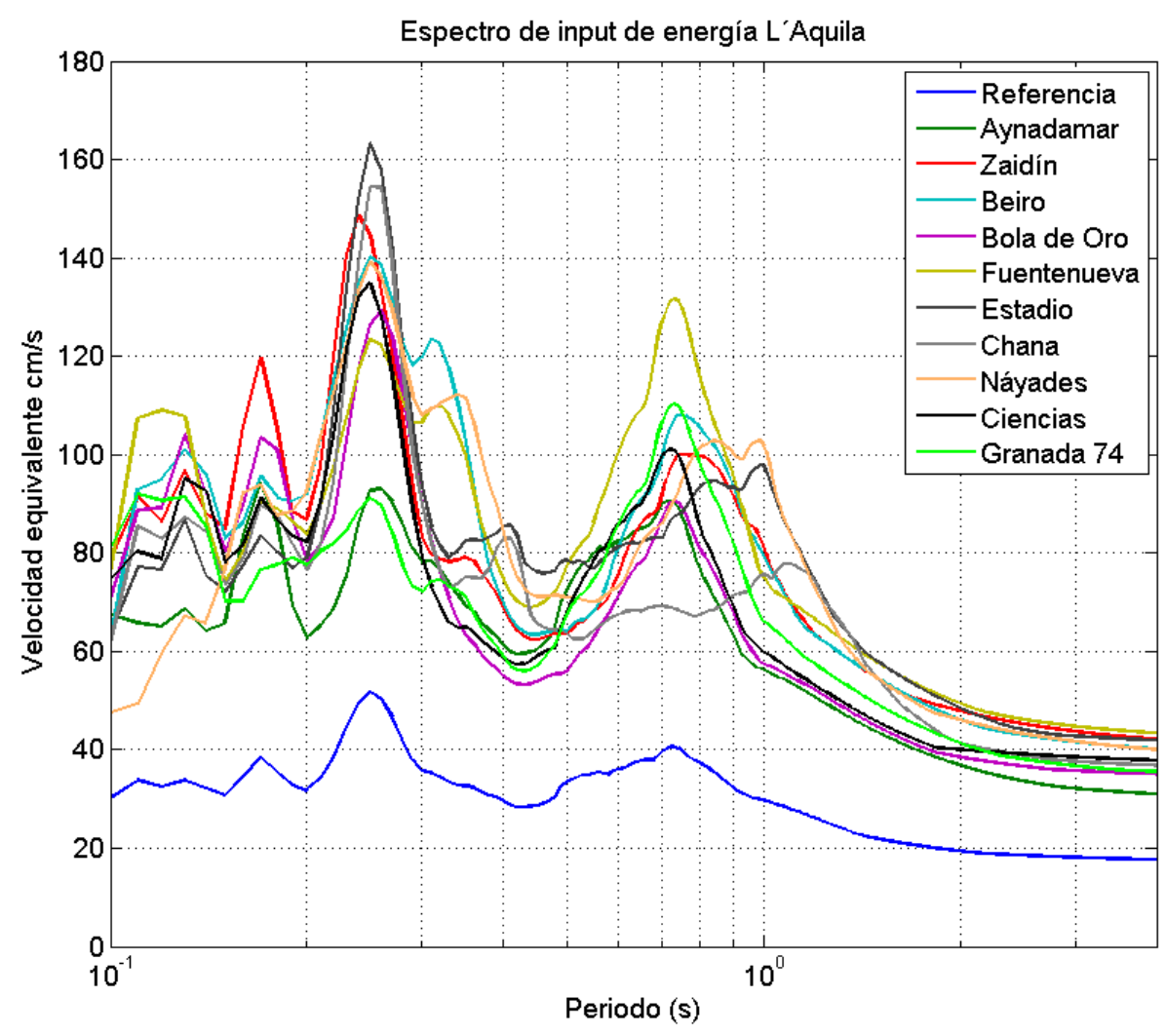

Figura 5.11 Espectros de input de energía del terremoto de L'Aquila y el de referencia. Obsérvese la gran diferencia entre los valores en suelo (10 sitios ) y en roca (el de referencia). 


\section{Conclusiones.}

\subsection{Conclusiones.}

A lo largo de presente trabajo se estudió el efecto que tiene la geología local de la ciudad de Granada en la modificación de las ondas sísmicas de potenciales terremotos en la zona. Debido a que la ciudad se encuentra en una zona de sismicidad moderada se ha recurrido a técnicas pasivas para caracterizar la respuesta dinámica local. Se han optado por las técnicas de SPAC y HVSR para encontrar modelos de suelo cuyas características sean capaces de reproducir la respuesta de los puntos estudiados. Mediante la inversión conjunta de las curvas de dispersión y curvas HVSR se han encontrado modelos de velocidad que a la postre han sido usados para simular la respuesta de sitio para terremotos reales ocurridos en otras zonas con características sismogenéticas similares a las de la ciudad de Granada.

Uno de los principales resultados obtenidos son los periodos o frecuencias predominantes de 17 puntos de la ciudad de granada a partir del método de Nakamura. Dichos periodos predominantes pueden ser usados en la mejora de la microzonificación sísmica de la ciudad. Como p.e. la hecha por Cheddadi (2001) que por la capacidad limitada del equipo de medición (en comparación al equipo usado en el presente trabajo) pudo haber omitido información por debajo de $1 \mathrm{~Hz}$.

La veracidad del cálculo de dichos periodos predominantes ha sido corroborada al hacer mediciones en tres sitios cercanos entre sí. Los sitios de la Calle Náyades, Calle Atlanta y Calle Carmen Amaya se distancian no más de 200 metros y su periodo predominante calculado ha sido de 1.1 s., 1.1 s., y 1.2 s. respectivamente. La poca variabilidad de los resultados en estos tres putos da garantía de que son las características de la geología local las que se han medido con la técnica de Nakamura.

Se ha llevado a cabo un test de Estacionaridad para estimar la ventana óptima de duración del registro de microtremor a fin de calcular la razón HVSR. Dicho test indica que periodos muy largos de medición deben ser considerados si se desean evitar frecuencias espúreas en los resultados. Se estudiaron dos sitios con condiciones de suelo distintas. El Sitio de Calle Náyades, cercano a la Vega de Granada, consta de suelos con rellenos de material aluvial antiguo, mientras que el sitio Calle Aixa la Horra se ubica sobre la Formación Alambra (Figura 2.7). Los resultados revelan que son necesarias ventanas de 60 minutos para el sitio con suelo 
blando y 90 minutos para el sitio de suelo consolidado. Periodos de medición como los anteriores satisfacen la determinación del periodo predominante a cualquier hora del día y a pesar de contar con fuentes cercanas de ruido indeseable como p.e. saltos de pavimento.

Se han logrado obtener modelos de velocidad a partir de la inversión conjunta de las curvas de dispersión y las curvas HVSR que son capaces de caracterizar la respuesta dinámica que tendrán los sitios de medición ante una posible sacudida sísmica. Los modelos de velocidad han llegado a profundidades cercanas a los 100 metros, lo que mejora la capacidad que por sí solas tienen las curvas de dispersión usadas en este trabajo. Dicha mejora se da por la adición de la información proveniente de la curva HVSR que obliga a los modelos generados por el método de inversión a reproducir tanto la curva de dispersión como la frecuencia predominante de la curva HVSR. Modelos de sitios cercanos a la formación Alhambra como Aynadamar y Bola de Oro constan de valores superiores de Vs para todos los estratos en comparación con aquellos sitios cercanos a la Vega de Granada, como lo son Náyades, Estadio y Chana. La diferencia en velocidad de las capas de los modelos y su relación con la información geológica de la ciudad dan certeza a las mediciones de microtremor y al proceso de inversión.

Se calcularon parámetros de movimiento sísmico para caracterizar la gravedad de una eventual sacudida sísmica. Los valores de PGA, PGV, CAV y Al han sido claramente mayores en los sitios de estudio que en la referencia ubicada sobre roca, p.e. el valor de Intensidad Arias es 7 veces superior en el sitio Náyades en comparación al de la referencia. Se encontró que en sitios cuyos modelos cuentan con una Vs relativamente baja, los valores de los parámetros vectoriales tienden a ser significativamente mayores a aquellos sitios con modelos cuya Vs es superior. Como muestra es el cálculo del PGV del terremoto de C-L, en donde Náyades, Estadio y Chana muestran una y media veces el valor alcanzado por Aynadamar y Bola de Oro.

Por otro lado se han obtenido espectros de respuesta de aceleración y espectros de input de energía en donde se observa una significativa diferencia entre los valores espectrales de los puntos de estudio los valores de la referencia. Los espectros de respuesta de aceleración de los sitios de suelo aumentan por lo menos el doble del valor del registro en roca para todo el rango de periodos estudiado. Se observan además picos de hasta el cuádruple del valor de la referencia en periodos que varían de acuerdo al sitio. Para un periodo cercano a los 0.2 segundos Náyades, Granada 74 y Beiro aumentan el triple del valor de referencia del terremoto de C-L, mientras que Chana y Ciencias llegan al cuadruplicarlo en un periodo 
cercano a 1.1 segundos. En cuanto a los espectros de input de energía el rango de periodos en donde se aprecia una amplificación de más del doble del valor de referencia va de 0.1 a 1.2 segundos. Presentándose para varios sitios picos múltiples de velocidad, p.e. el caso de Beiro en donde existen picos a $0.2,0.3$ y 0.9 segundos que triplican el valor del registro en roca del terremoto de C-L. Con lo que se concluye que la geología local de la ciudad de Granada es capaz de alterar las características espectrales de las ondas sísmicas de manera importante y arbitraria.

Los métodos pasivos aquí estudiados han demostrado tener la capacidad de extraer información relevante sobre la estructura de suelo de una ciudad como Granada. Con la implementación de una inversión conjunta de las curvas de dispersión y curvas HVSR se pude caracterizar la respuesta sísmica del suelo con todos los beneficios que conlleva usar como fuente de información el ruido sísmico ambiental.

\subsection{Recomendaciones para trabajos futuros.}

Con el fin de mejorar la información acerca de los efectos de sitio presentes en la ciudad de Granada se propone aumentar el número de mediciones de microtremor para efectuar una microzonificación más detallada. Teniendo especial cuidado en utilizar equipo capaz de registrar frecuencias bajas, del orden de $0.5 \mathrm{~Hz}$, y tomando ventanas de tiempo adecuadas para el tipo de suelo presente y así poder localizar mediante la técnica de Nakamura los periodos predominantes reales del sitio. Se sugiere además mejorar la resolución del método SPAC con la implementación de modificaciones a la configuración del array como los propuestos por Chávez-García et al. (2005). 


\section{Referencias.}

Aki, K. (1957). Space and time spectra of stationary stochastic waves with special reference to microtremors, Bull. Erthq. Res. Ins. 35, 415-456.

Aki, K. (1993). Local site effects on weak and strong ground motion, Tectonophysics 218, 93111.

Aki, K., and P. G. Richards (1980). Quantitative Seismology: Theory and methods, W.H. Freeman, San Francisco.

Aki, K., and P. G. Richards (2002). Quantitative Seismology, Second Ed., University Science Books, Sausalito, California.

Akiyama, H. (1980). Earthquakes-resistance limit-state design for buildings, Universitu of Tokio Press, Tokio.

Al Yuncha, Z., Luzón, F. (2000). On the horizontal to vertical Spectral Ratio in Sedimentary Basins, Bull. Seism. Soc. Am. 90, 1101-1106.

Alguacil G., Vidal F., Navarro M., García-Jerez A. and Pérez-Muelas J (2012). Characterization of earthquake shaking severity at different sites of Lorca twn for the May 11th, 2011 event, Bull Earthquake Eng.

Alguacil, G., Vidal, F. (2012). Medidas Istrumentales de la intensidad del movimiento del suelo. Aplicacion a terremotos europeos, en Homenaje a la profesora María Dolores Romacho Romero, Editorial Universidad de Almería.

Arias, A. (1970). A measure of earthquake intensity, In: R. Hansen (Editor), Seismic Design for Nuclear Power Plant, MIT Press, Cambridge, Massachusetts.

Azañón, J.M., Booth Rea, G., Martínez Martínez, J.M., Pérez Peña, V. (2007). Actividad Tectónica durante la época medieval y romana en la Cuenca de Granada (la falla de la Malahá), Reunión Nacional del Cuaternario, Ávila 2007.

Bard, P.-Y. (1994). Effects of surface geology on ground motion: recent results and remaining issues, in Proc. 10th European Conf. on EarthquakeEngineering, Vienna, 305-323.

Beck, J. L. And Hall, J. F. (1986). Factors Contributing to the catastrophe in Mexico city during the earthquake of September 19, 1986, Geophysical Research Letters 13, 593-596.

Benavent-Climent, A. (2010). Estructuras sismorresistentes, Maia Ediciones. Madrid, España.

Bettig, B., Bard, P. Y., Scherbaum, F., Riepl, J., Cotton, F., Cornou, C., Hatzfeld, D. (2001). Analysis of dense array noise measurements using the modified spatial autocorrelations method (SPAC): Application to the Grenoble area, Bolletino Di Geofisica Teorica Ed Applicata 42, pp. 281-304.

Bonnefoy-Claudet, S., Cornou, C., Bard, P-Y., Cotton, F., Moczo, P., Kristek, J. and Fäh, D. (2006) $\mathrm{H} / \mathrm{V}$ ratio: a tool for site effects evaluation. Results from 1-D noise simulations. Geophys. J. Int. 167, 827-837.

Borcherdt, R. D. (1970). Effects of local geology on ground motion near San Francisco Bay, Bull. Seism. Soc. Am. 60, 29-61.

Borcherdt, R. D. (1990). Influence of local geology in the San Francisco Bay region, California on ground motions generated by Loma Prieta earthquake of October 17, 1989. Int. Symp. on Safety of Urban Life and Facilities. Lessons learned from the 1989 Loma Prieta Earthquake. November, 1990. Tokyo, 1-35.

Borcherdt, R. D., Glassmoyer, G., Andrews, M., Cranswick, E. (1989). Effect of site conditions on ground motion and damage, Earthquake Spectra (Special supplement). Armenia Earthquake Reconnaissance report. (eds. Wyllie L.A. and Filson J.R.), 23-42.

Cabañas, L., Benito, B., Herráiz, M. (1997). An approach to the measurement of the potential structural damage of earthquake ground motion, Earthquake Eng. Struct. Dyn. 26, 7992. 
Campillo, M., J. C. Gariel, K. Aki, and F. J. Sfinchez-Sesma (1989). Destructive strong ground motion in Mexico City: source, path, and site effects during great 1985 Michoacan earthquake, Bull. Seism. Soc. Am. 79, 1718-1735.

Celebi, M., J. Prince, C. Dietel, M. Onate, and G. Chavez (1987). The culprit in Mexico City-amplification of motions, Earthquake Spectra 3, 315-328.

Chávez-García, F. J. (1991). Diffraction et amplification des ondes sismiques dans le bassin de Mexico, Ph.D. Thesis. Universit Joseph Fourier de Grenoble, 331 pp.

Chávez-García, F. J., M. Rodríguez, and W. R. Stephenson (1999). Lateral propagation effects observed at Parkway, New Zealand: a case history to compare 1D versus 2D site effects, Bull. Seism. Soc. Am. 89, 718-732.

Chávez-García, F. J., M. Rodríguez, and W. R. Stephenson (2005). An alternative to the SPAC analysis of microtremors: exploiting stationarity of noise, Bull. Seism. Soc. Am. 95, 277293.

Chávez-García, F. J., M. Rodríguez, and W. R. Stephenson (2006). Subsoil Structure Using SPAC Meadurements along a Line, Bull. Seism. Soc. Am. 96, 729-736.

Cheddadi, A. (2001). Caracterización sísmica del subsuelo de la ciudad de granada mediante análisis espectrales del ruido de fondo sísmico. Tesis Doctoral. Universidad de Granada.

EPRI (1988). A criterion for determining exceedance of the operating basis earthquake, EPRI NP-5930, Electrical Power Research Inst., Palo Alto, CA.

Feriche Fernández-Castanys, M. (2012). Elaboración de escenarios de daños sísmicos en la ciudad de Granada. Tesis Doctoral. Universidad de Granada.

Finn, W. D. L. (1991). Geotechnical engineering aspects of microzonation, Proc. Fourth Int. Conf. on Seismic Zonation, Stanford, California, I, 199-259.

García-Jeréz, A. (2010). Desarrollo y evaluación de métodos avanzados de exploración sísmica pasiva. Tesis Doctoral. Universidad de Almeria.

Goldstein, P., and R. J. Archuleta (1987). Array analysis of seismic signals, Geophys. Res. Lett. 14, 13-16.

Gutenberg, B. (1958). Microseisms. Advances in Geophysics 5, 53-92.

Haertzell, S. H. (1978).Earthquake aftershocks as Green's functions, Geophys. Res. Lett., 5, 1-4.

Haskell, N. A.(1953). The dispersion of surface waves on multilayered media, Bull. Seism. Soc. Am., 43, 17-34.

Herak, M. (2008). ModelHVSR-A Matlab tool to model horizontal to vertical spectral ratio of ambient noise, Computers and Geociences 34, 1514-1526.

Hernández del Pozo (1998). Análisis metodológico de la cartografía geotécnica urbana, aplicación a la ciudad de Granada. Tesis Doctoral. Universidad de Granada.

Kagawa T. and Group for Spanish-Japanese Joint Work on Microzonation of Granada Basin (1999). Micreotremor Array Observation in the Granada Basin, Southern Spain, In Joint Study on Seismic Microzonation in Granada Basin, Spain, 64-81.Tokyo Institute of Technology.

Kanai, K., T. Tanaka, T. Morishita and K. Osada (1966). Observation of microtremors, XI (Matsushiro earthquake swarm area), Bull. Earthquake Res. Inst. 44, 1297-1333.

Konno K., Ohmachi, T. (1998). Ground motion characteristics estimated from spectral ratio between horizontal and vertical components of microtremors. Bull. seism. Soc. Am., 88-1, 228-241.

Lermo, J. (1992). Observaciones de microtremores en México y su aplicaci6n en la Ingeniería Sísmica, MS Thesis, Universidad Nacional Autónoma de México.

Lermo, J., and Chávez-García, F. J. (1993). Site effect evaluation using spectral ratios with only one station, Bull. Seism. Soc. Am. 83, 1574-1594. 
Lermo, J., and F. J. Chávez-García (1994). Are microtremors useful in site esponse evaluation? Bull. Seism. Soc. Am. 84, 1350-1364.

Lim, J.S., Openheim, A.V. (Ed.) (1988): Advanced Topics in Signal Processing. Prentice Hall.

Luzón, F., Al Yuncha, Z., Sánchez-Sesma, F. J. And Otiz-Alemán, C. (2001). A numerical experiment on the Horizontal to Vertical Spectral Ratio in flat sedimentary basins, Pure appl. Geophys. 158, 2451-2461.

Luzón, F., Palencia, V. J., Morales, L., Sánchez-Sesma, F. J., García, J. M. (2002). Evaluation of the site effects in sedimentary Basins, Física de la Tierra 14, 183-214.

Martínez-Rueda, J.E. and Tsantali, E. (2008). Analysis of the correlation between instrumental intensities of strong earthquake ground motion, In Eurodyn 2008, $7^{\text {th }}$ European Conference on Structural Dynamics, Southampton.

Nakamura, Y. (1989). A method for dynamic characteristics estimation of subsurface using microtremor on the ground surface, Q. Rept. Railway Tech. Res. Inst. 30, 25-33.

Navarro, M., García-Jerez, A., Vidal, F., Azañon, J. M., Feriche, M., Enomoto, T. (2010). Vs30 Structure of Granada town (southern Spain) from the Ambient Noise Array Observations. In 14th European Conference on Earthquake Engineering. Ohrid, Republic of Macedonia. Septiembre de 2010.

NCSE-02 (2002) Normativa de Construcción Sismorresistente Española. Real Decreto 997/2002. Boletín Oficial del Estado No. 244, 11 Octubre, 2002, España.

Ohmachi, T., Y. Nakamura, and T. Toshinawa (1991). Ground motion characteristics in the San Francisco Bay area detected by microtremor measurements, Proc. 2nd. Int. Conf. on Recent Advances in Geotech. Earth. Eng. \& Soil Dyn., San Louis, Missouri, 11-15 March, 1643-1648.

Ordaz M, Montoya C (2012) Programa DEGTRA A4 versión 9.1.0 Instituto de Ingeniería, Universidad Nacional Autónoma de México, México.

Press, W.H., Flannery, B.P., Teukolsky, S.A., Vetterling, W.T. (1989): Numerical Recipes. The Art of Scientific Computing (Fortran version). Cambridge University Press.

Richter, Charles F. (1958). Elementary Seismology, W. H. Freeman and Company, Inc., San Francisco, 768.

Rodríguez, F.J., Sanz de Galdeano, C. (2006). Late orogenic intramontane basin development: the Granada basin, Betics (southern Spain), Basin Research 18, 85-102.

Sadik, B.B., Sucuoglu, H., Yilmaz, T. (2002). An overview of Local Site Effects and the Associated Building Damage in Adapazari during the 17 Agust 199 Izmit Earthquake, Bull. seism. Soc. Am., 92, 509-526.

Sambridge, M. (1999). Geophysical inversion with a neighbourhood algorithm I. Searching a parameter space, Geophysical Journal International 103, 4839-4878.

Sánchez-Sesma, F. J. (1987). Site effects on strong ground motion, Int.J. Soil Dynamics and Earthquake Engineering 6, 124-132.

Sánchez-Sesma, F. J., Rodríguez, M., Iturrarán-Viveros, U., Luzón, F., Campillo, M., Margerin, L., García-Jerez, A., Suarez, M., Santoyo, M.A., and Rodríguez-Castellanos A. (2011). A theory for microtremor $\mathrm{H} / \mathrm{V}$ spectral ratio: Application for a layered medium, Geophys. J. Int. 186, 221-225.

Sanz de Galdeano, C., Pelaez, J.A. and Lopez-Casado, C. (2003). Seismic potentiality of the main active faults in the Granada Basin (South of Spain), Pure and Applied Geophysics, 160,1537-1556.

Sanz de Galdeano, C., Peláez, J.A. y López-Garrido, A.C. (Eds.). (2001). La cuenca de Granada. Estructura, Tectónica Activa, Sismicidad, Geomorfología y dataciones existentes. CSICUniv. Granada. ReproDigital. 218 pp. 
Scherbaum, F., Hinzen, K.-G., Ohrnberger, M. (2003). Determination of shallow shear wave velocity profiles in the Cologne/Germany area using ambient vibrations, Geophys. J. Int., 152, 597-612.

Singh, S. K., Mena, E., Castro, R. (1988). Some aspects of source characteristics of the 19 September, 1985, Michoacán Earthquake and ground motion amplification in and near Mexico City from strong ground motion data, Bull. Seism. Soc. Am., 78, 451-477.

SISMOSAN (2007). Proyecto de "Peligrosidad y Riesgo Sísmico en Andalucía". Informe interno realizado para la Consejería de Gobernación de la Junta de Andalucía. 5 Volúmenes.

Somerville, P., (1995). Geotechnical reconnaissance of the effects of the january 17, 1995, Hyogo-ken Nanbu earthquake, Japan. Technical Report UCB/EERC-95/01, Earthquake Engineering Research Center, University of California, Berkely, California.

Taga, N. (1993). Earthquake motion and ground conditions, (Ed. The Architectural Institute of Japan), Tokyo. pp. 315-325.

Thomson, W. T.( 1950). Transmission of elastic waves through a stratified solid medium, Journal of Applied Physics, 21, 89-93.

Toki, K., Irikura, K., Kagawa, T. (1995). Strong motion data recorded in the source area of the 1995 Hyogoken-nanbu earthquake, January 17, 1995 Japan. J. Natural Disaster Sci. 16 (2), 23-30.

Udwadia, F. E. and Trifunac, M. D. (1973). Comparison of earthquake and microtremor ground motions in El Centro, California, Bull. Seism. Soc. Am. 63, 1227-53.

Valverde, I. (2010) Cimentaciones de edificios en condiciones estáticas y dinámicas: casos de estudio al Oeste de la ciudad de Granada. Tesis Doctoral. Universidad de Granada.

Vazquez, R. R., Aguirre, J., Flores, E. H-., Mijares, A. H. (2011). Microtremor studies using the SPAC method: Experiences and applications to four sites in Mexico, Geofís. Intl. 50, 295-312.

Vidal, F. (1986). Sismotectónica de la región Béticas-Mar de Alborán. Tesis Doctoral. Universidad de Granada.

Vidal, F., Feriche, M., Navarro, M., Ruíz A. (2010). Earthquake Damage Scenarios in Granada Town (Southern Spain). In 14th European Conference on Earthquake Engineering. Ohrid, Republic of Macedonia. Septiembre de 2010.

Wald, D. J., Quitoriano, V., Heaton, T. H., and Kanamori, H. (1999). Relationships between peak ground acceleration, peak ground velocity and Modified Mercalli Intensity in California, Earthquake Spectra, 15, 557-564.

Wathelet, M. (2007). Array recordings of ambient vibrations: surface wave inversion. Ph.D. Thesis. Université de Liege.

Wathelet, M., D. Jongmans, and M. Ohrnberger (2004). Surface wave inversion using a direct search algorithm and its application to ambient vibration measurements, Near Surface Geophysics 2, 211--221.

Wathelet, M., D. Jongmans, and M. Ohrnberger (2005). Direct Inversion of Spatial Autocorrelation Curves with the Neighborhood Algorithm, Bull. seism. Soc. Am., 95, 1787-1800.

Worden, C. B., Gerstenberger, M. C., Rhoades, D. A., Wald, D. J. (2012). Probabilistic Relationships between Ground Motion Parameters and Modified Mercalli Intensity in California, Bull. Seism. Soc. Am., 102, 204-221. 


\section{Anexo A: Programa HVSR PWELCH}

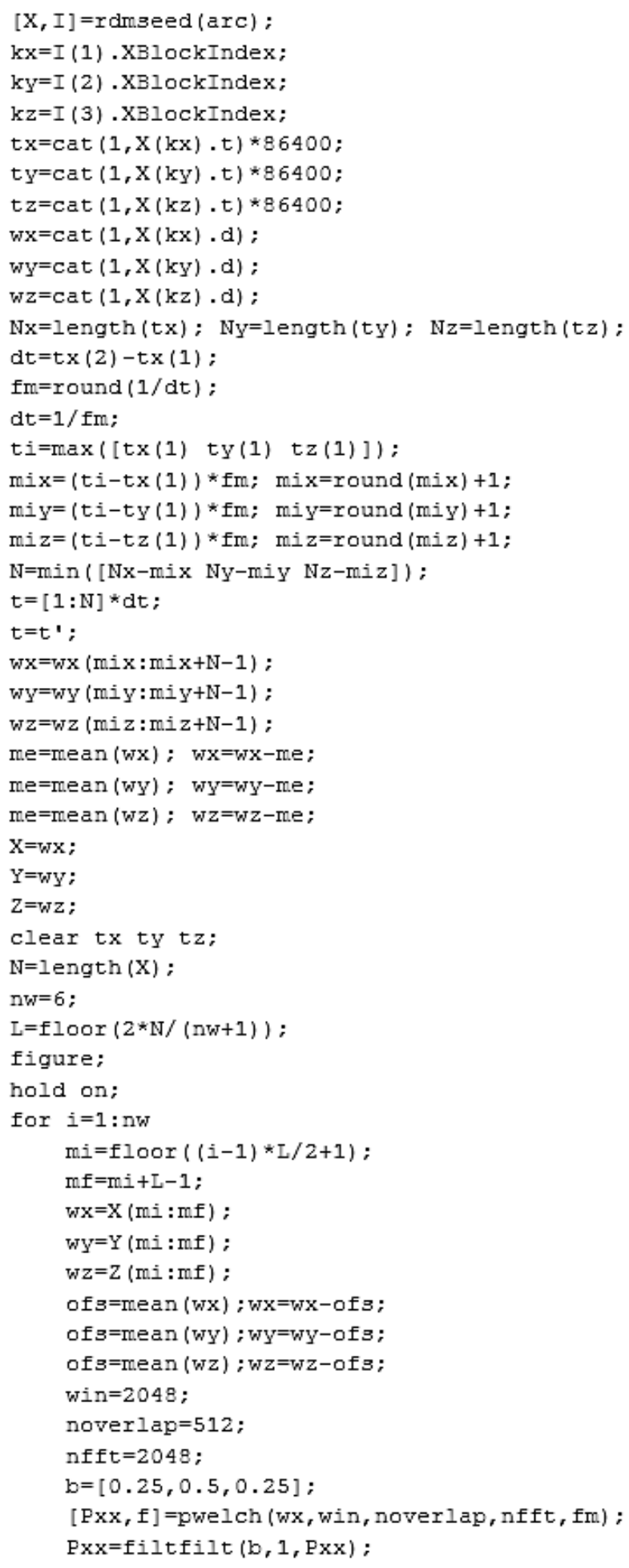


[PYY, f] =pwelch (wY, win, noverlap, nfft, fm) ; PyY=filtfilt $(b, 1$, PYY $)$;

$[P z z, f]=p w e l c h(w z, w i n$, noverlap, nfft, fm) ;

Pzz=filtfilt $(b, 1, P z z)$;

$\mathrm{hv}=3 \mathrm{qrt}(((\mathrm{Pxx}+\mathrm{Pyy}) / 2) . / \mathrm{Pzz})$;

HV $(:, i)=h v$;

$\operatorname{plot}(\mathrm{f}, \mathrm{hv})$;

end

$\mathrm{LHV}=\log 10$ (HV) ;

LHVm=mean $($ LHV , 2) ;

LHVs=std $($ LHV $, 1,2)$;

HVm $=10 \cdot{ }^{\wedge} \mathrm{LHVm}$;

HVs1 $=10 \cdot \wedge(\mathrm{LHVm}+\mathrm{LHV} s)$;

HVs $2=10 . \wedge($ LHVm-LHVs $)$;

figure

semilogx (f, HVm, f, HVs1, f, HVs2, 'LineWidth' , 2) ;

legend ('HVSR', '+1DS', '-1DS');

xlim([0.5 10]);

ylim([0 10]) ;

xlabel ('Frecuencia (HZ)');

ylabel ('Amplitud H/V');

grid on:

$\mathrm{HVSR}=\mathrm{HVm}$;

$\mathrm{fHV}=\mathrm{f}$;

save ('HVSRFwelch', 'HVSR', 'fHV'); 


\section{Anexo B: Programa HVSR MEM}

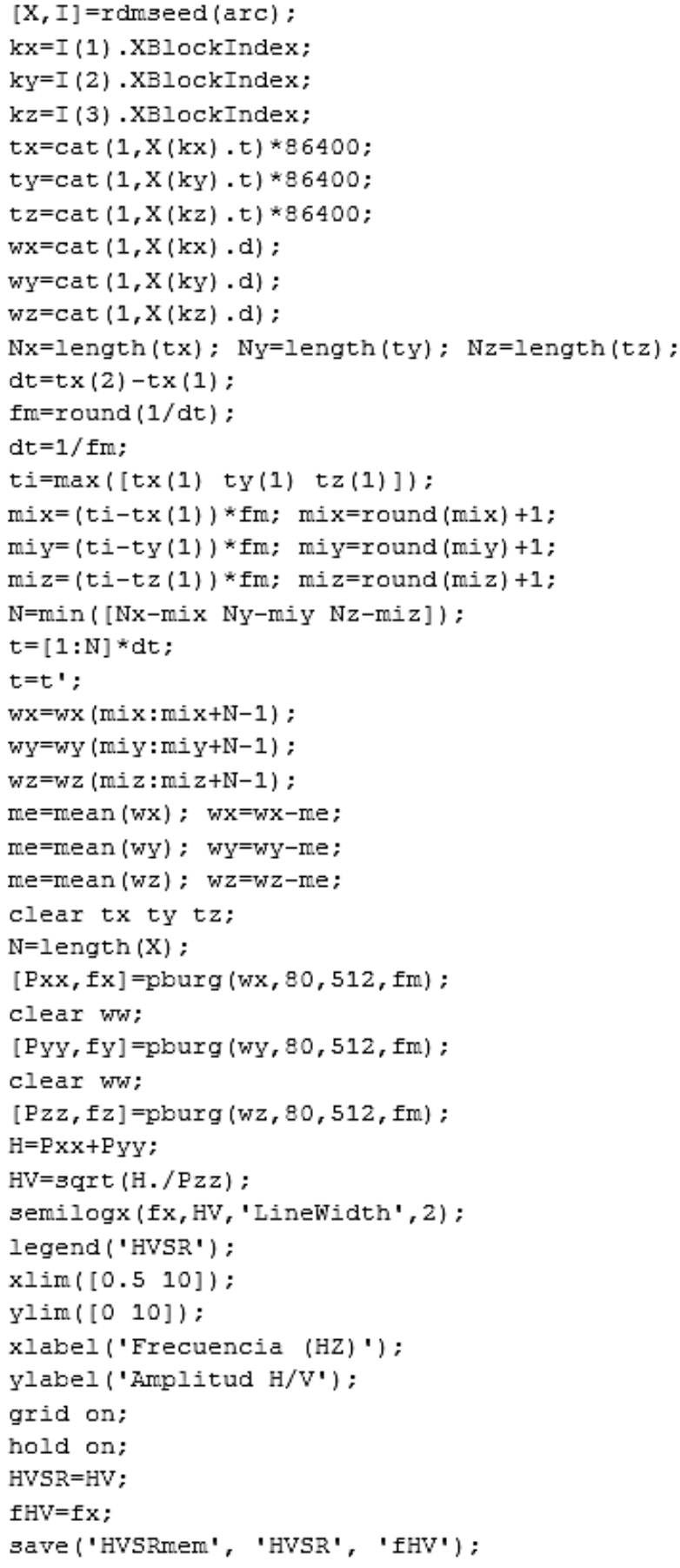

